\title{
Generalized Orbifold Construction for Conformal Nets
}

\author{
MARCEL BISCHOFF
}

Dedicated to the memory of John E. Roberts

\begin{abstract}
Let $\mathcal{B}$ be a conformal net. We give the notion of a proper action of a finite hypergroup acting by vacuum preserving unital completely positive (so-called stochastic) maps, which generalizes the proper actions of finite groups. Taking fixed points under such an action gives a finite index subnet $\mathcal{B}^{K}$ of $\mathcal{B}$, which generalizes the $G$-orbifold. Conversely, we show that if $\mathcal{A} \subset \mathcal{B}$ is a finite inclusion of conformal nets, then $\mathcal{A}$ is a generalized orbifold $\mathcal{A}=\mathcal{B}^{K}$ of the conformal net $\mathcal{B}$ by a unique finite hypergroup $K$. There is a Galois correspondence between intermediate nets $\mathcal{B}^{K} \subset \mathcal{A} \subset \mathcal{B}$ and subhypergroups $L \subset K$ given by $\mathcal{A}=\mathcal{B}^{L}$. In this case, the fixed point of $\mathcal{B}^{K} \subset \mathcal{A}$ is the generalized orbifold by the hypergroup of double cosets $L \backslash K / L$.

If $\mathcal{A} \subset \mathcal{B}$ is an finite index inclusion of completely rational nets, we show that the inclusion $\mathcal{A}(I) \subset \mathcal{B}(I)$ is conjugate to a Longo-Rehren inclusion. This implies that if $\mathcal{B}$ is a holomorphic net, and $K$ acts properly on $\mathcal{B}$, then there is a unitary fusion category $\mathcal{F}$ which is a categorification of $K$ and $\operatorname{Rep}\left(\mathcal{B}^{K}\right)$ is braided equivalent to the Drinfel'd center $Z(\mathcal{F})$. More generally, if $\mathcal{B}$ is completely rational conformal net and $K$ acts properly on $\mathcal{B}$, then there is a unitary fusion category $\mathcal{F}$ extending $\operatorname{Rep}(\mathcal{B})$, such that $K$ is given by the double cosets of the fusion ring of $\mathcal{F}$ by the Verlinde fusion ring of $\mathcal{B}$ and $\operatorname{Rep}\left(\mathcal{B}^{K}\right)$ is braided equivalent to the Müger centralizer of $\operatorname{Rep}(\mathcal{B})$ in the Drinfel'd center $Z(\mathcal{F})$.
\end{abstract}

\section{Contents}

1. Introduction

2. Prelimaries

3. Hypergroup Actions on Conformal Nets

4. Hypergroup Actions from Subfactors and Conformal Subnets

5. Commutative Q-systems in Unitary Modular Tensor Categories and Inclusions of Completely Rational Conformal Nets

6. Possible Generalization to Infinite Actions

\section{INTRODUCTION}

For (rational) chiral conformal theory, there are two main axiomatizations: conformal nets and vertex operator algebras. In both frameworks there is a notion of finite conformal inclusions, (finite) extensions and subtheories. Both settings have a form of rationality in which the representation categories are modular tensor categories. In this case chiral extensions and their representation theory is well understood through commutative algebra objects (called Q-systems for nets) in the representation category and dyslexic modules (called ambichiral sectors for nets), respectively, see

Date: November 12, 2018.

Supported by NSF Grant DMS-1362138. 
[KO02, HKL15 for VOAs and [LR95, Müg10, BKL15 for conformal nets. A model independent understanding of subtheories exists only in the case of fixed points with respect to a finite group $G$, so-called $G$-orbifolds, see [DM97] for VOAs and [Xu00] for conformal nets. Nevertheless, the structure is already very interesting in this setting. It leads to the theory of twisted representation and $G$-crossed braided tensor categories [Müg05]. The present paper tries to fill the gap by introducing a model independent theory of more general fixed points.

Exotic subfactors and fusion categories lead to new modular tensor categories via the quantum double construction and there is some indication that these (maybe all) are realized by finite index subnets of holomorphic nets (= conformal nets with trivial representation category) [Bis16b]. The first idea is to look into finite index subnets of already constructed conformal nets. E.g. Evans and Gannon give indication that there should be a subtheory of the chiral theory associated with the $A_{2} \times E_{6}$ lattice (which embeds into the holomorphic $E_{8}$ theory) which should give the double of the Haagerup subfactor as a representation category. We mention that the study of conformal inclusions/embeddings [SW86] which were studied in the framework of conformal nets in [Xu98b, $\mathrm{Xu} 98 \mathrm{a}$, gives many examples of finite index subnets. But given a conformal net $\mathcal{B}$ a general theory and characterization of finite index subnets $\mathcal{A} \subset \mathcal{B}$ has not been established. Related to this, Evans and Gannon [EG11] asked if one can orbifold a holomorphic net by something more general than a group.

The goal of this paper is to define a generalized notion of an orbifold, which cover all finite conformal inclusions. This should be a generalizing of the fixed point by a finite group, a so-called $G$-orbifold. Such a $G$-orbifold is given by automorphisms $\left\{\alpha_{g} \in \operatorname{Aut}(\mathcal{A})\right\}_{g \in G}$ of vacuum preserving automorphisms of the net.

In our approach groups are generalized to hypergroups and actions by vacuum preserving automorphisms to actions by stochastic maps.

Stochastic maps are unital completely positive maps preserving a state and arise in the study of non-commutative probability spaces. A non-commutative probability space is a pair $(M, \varphi)$ of a von Neumann algebra $M$ and a faithful normal state $\varphi$. In particular, every local algebra $\mathcal{A}(I)$ together with its vacuum state $\varphi=(\Omega, \cdot \Omega)$ is a non-commutative probability space.

We remark that a subfactor $\mathcal{A}(I) \subset \mathcal{B}(I)$ itself can be seen as a generalization of a group fixed point, but given a net $\mathcal{A}(I)$ there is no indication to see when a subfactor $N \subset \mathcal{A}(I)$ comes from a subnet. Further, we point out that a phenomenon of decategorification occurs. This already occurs in the case when we have a $G$-orbifold of a holomorphic net. Namely, we get a class $[\omega] \in H^{3}(G, \mathbb{T})$, which is exactly the data which gives a categorification of $G$ as a unitary fusion category. But the action of $G$ itself does not involve $[\omega] \in H^{3}(G, \mathbb{T})$. In general, in the holomorphic case we show that we get a hypergroup acting and that we get a categorification in terms of a unitary fusion category.

We point out that there is a proposal to use defects to study generalized orbifolds, [FFRS10]. But there the point is that if we have a chiral theory $\mathcal{A} \subset \mathcal{B}$ we can get an associated full conformal field theory by knowing the correlators of e.g. the Cardy case full conformal field theory of $\mathcal{A}$. In particular, the knowledge of $\mathcal{A}$ is already assumed. Defects can be defined for conformal nets on the line as in BKLR16 and there is a connection between the action of the stochastic map and the physical behaviour of the defect. Namely, let $\mathcal{A} \subset \mathcal{B}$ and consider all $\mathcal{A}$-topological $\mathcal{B}$ - $\mathcal{B}$ defects. Then $\mathcal{A}$ can be characterized to be the maximal subnet of $\mathcal{B}$, which is invisible for all $\mathcal{B}-\mathcal{B}$ defects. By identifying the left and right copy of $\mathcal{B}$ we get an action on the observables of $\mathcal{B}$ as in BKLR16. Sec 5.4]. Our approach to generalized orbifolds presented in this paper are based on this observation. We will present the relationship between generalized orbifolds presented here and phase boundaries in [BKLR16] in a future publication.

The main results. We introduce the following subfamilies of conformal nets:

$\{$ holomorphic nets $\} \subset\{$ quantum double nets $\} \subset\{$ completely rational nets $\} \subset\{$ conformal nets $\}$ 
By conformal net $\mathcal{A}$ we mean a Möbius covariant local and irreducible net on the circle $\mathbb{S}^{1}$. It is completely rational, if it has finite $\mu$-index, is strongly additive, and fulfills the split property. In this case, $\operatorname{Rep}(\mathcal{A})$ is a unitary modular tensor category [KLM01]. If it happens to be the trivial category (which is equivalent with the $\mu$-index $\mu(\mathcal{A})$ begin equal to 1 ), then we call $\mathcal{A}$ a holomorphic net. If $\mathcal{A} \subset \mathcal{B}$ a finite index inclusion $([\mathcal{B}: \mathcal{A}]<\infty)$ and $\mathcal{B}$ holomorphic, we call $\mathcal{A}$ a quantum double net. This property is equivalent to $\operatorname{Rep}(\mathcal{A})$ being braided equivalent to the unitary Drinfel'd center also called quantum double $Z(\mathcal{F})$ |Müg03b of a unitary fusion category $\mathcal{F}$ (see e.g. [Müg10, Bis16b|).

Proposition 1.1 (see Corollary 4.2). Let $\mathcal{A} \subset \mathcal{B}$ be a finite index subnet, then the canonical endomorphism $\gamma \in \operatorname{End}(\mathcal{B}(I))$ of the inclusion $\mathcal{A}(I) \subset \mathcal{B}(I)$ has no multiplicities, i.e. $\operatorname{Hom}(\gamma, \gamma)=$ $\gamma(\mathcal{B}(I))^{\prime} \cap \mathcal{B}(I)$ is a commutative algebra.

One might wonder if in this case $\mathcal{A}(I) \subset \mathcal{B}(I)$ could be seen as the fixed points by an outer action of a Hopf algebra. This would mean that the inclusion $\mathcal{A}(I) \subset \mathcal{B}(I)$ has depth 2. This is indeed the case if and only if it is a group fixed point.

Corollary 1.2. If $\mathcal{A}(I) \subset \mathcal{B}(I)$ is finite index and depth 2, or equivalently fixed point by a Kac algebra, then it is a group fixed point.

Proof. For depth 2 we have $[\gamma]=\bigoplus_{i} n_{i}\left[\beta_{i}\right]$ with $d \beta_{i}=n_{i}$ Lon94, but from Proposition 1.1 follows that $\gamma$ has no multiplicities and therefore $d \beta_{i}=1$ and the statement follows from Izu91, Theorem 4.1] or the fact that the hypergroup $K$ in Proposition 1.10 is indeed a group.

In Section 3 (see Definition 3.1 and 3.7) we define a proper action of a hypergroup $K$ on a conformal net $\mathcal{B}$, which generalizes the action of a finite group $G$ by inner symmetries. A hypergroup is a finite set $K=\left\{c_{0}, \cdots, c_{n}\right\}$ which is the basis of a $*$-algebra fulfilling certain axioms (see Definition 2.3). Each element $c_{i}$ has a weight $w_{i}=w_{c_{i}} \geq 1$ and $K$ is a finite group if and only if $w_{i}=1$ for all $c_{i} \in K$. The weight of a hypergroup $K$ is defined to be $D(K)=\sum_{i=0}^{n} w_{i}$, so in particular $D(G)=|G|$ for a finite group $G$.

Theorem 1.3 (see Theorem 3.8 and 4.22 . Let $\mathcal{B}$ be a conformal net.

(1) Let $K$ be a hypergroup acting properly on $\mathcal{B}$, then the fixed point net $I \mapsto \mathcal{B}(I)^{K}$ turns out to be a finite index subnet of $\mathcal{B}$, called the $K$-orbifold net. The index $\left[\mathcal{B}: \mathcal{B}^{K}\right]$ equals $D(K)$.

(2) Conversely, let $\mathcal{A} \subset \mathcal{B}$ be a finite index subnet, then there is a canonical (and unique up to equivalence) proper hypergroup action of a hypergroup $K$ on $\mathcal{B}$, such that $\mathcal{A}=\mathcal{B}^{K}$. This construction recovers the action of $K$ from (1) up to equivalence.

(3) There is a Galois correspondence between intermediate nets $\tilde{\mathcal{A}}$ with $\mathcal{B}^{K} \subset \tilde{\mathcal{A}} \subset \mathcal{B}$ and subhypergroups $L \subset K$. In this case $\tilde{\mathcal{A}}=\mathcal{B}^{L}$ and there is a canonical action of the hypergroup of double cosets $K / / L$ on $\tilde{\mathcal{A}}$, such that $\mathcal{A}=\tilde{\mathcal{A}}^{K / / L}$.

Let us from know on suppose that $\mathcal{B}$ is completely rational (then every finite index subnet $\mathcal{A} \subset \mathcal{B}$ is completely rational by Lon03]). In this case we get a complete characterization of how the actions of hypergroups look like.

We have the following categorical result.

Proposition 1.4 (Proposition 5.5). Let $\Theta$ be a Lagrangian Q-system in a UMTC C , and $\mathcal{D} \cong{ }_{\Theta} \mathcal{C}_{\Theta}$ the dual category. Then the dual $Q$-system $\Gamma \in \mathcal{D}$ is the Longo-Rehren Q-system associated with $\mathcal{C}_{\Theta} \cong \mathcal{D}^{+}$.

In the case $\mathcal{B}$ is even holomorphic we first get the following Corollary of Propopsition 1.4 .

Corollary 1.5. Let $\mathcal{A} \subset \mathcal{B}$ be a finite index subnet, $\mathcal{B}$ holomorphic, then the dual Q-system of the inclusion $\mathcal{A}(I) \subset \mathcal{B}(I)$ is a Longo-Rehren Q-system, in other words $\mathcal{A}(I) \subset \mathcal{B}(I)$ is isomorphic to a Longo-Rehren inclusion. 
There is the following natural open question which is also related to the question if all finite index finite depth subfactors come from conformal nets Bis16a.

Question 1.6. Let $\mathcal{F}$ be a unitary fusion category. Is there a completely rational net $\mathcal{A}$, such that $\operatorname{Rep}(\mathcal{A})$ is braided equivalent to the (unitary) Drinfel'd center $Z(\mathcal{F})$ ?

The following theorem says that such a net $\mathcal{A}$ is always a generalized orbifold of a holomorphic net. The special case of the theorem where $K=G$ is a finite group was announced by Müger [Müg10, 3.6 Corollary] with the missing proofs contained in the categorical work [DMNO13]. Namely, for a holomorphic net $\mathcal{B}$ and a finite group $G \subset \operatorname{Aut}(\mathcal{B})$ (i.e. a proper action of $G$ on $\mathcal{B}$ ) we have $\operatorname{Rep}\left(\mathcal{B}^{G}\right) \cong$ $\operatorname{Rep}\left(D^{\omega}(G)\right) \cong Z\left(\operatorname{Vect}_{G}^{\omega}\right)$ for some $[\omega] \in H^{3}(G, \mathbb{T})$ and conversely, if $\operatorname{Rep}(\mathcal{A}) \cong Z\left(\operatorname{Vect}_{G}^{\omega}\right)$ then there is a holomorphic net $\mathcal{B}$ with $\mathcal{A}=\mathcal{B}^{G}$. Our analogous but much more general result using generalized orbifolds is:

Theorem 1.7 (holomorphic case, see Theorem 5.16). Let $\mathcal{B}$ be a holomorphic conformal net with a proper action of a hypergroup $K$. Then there is a unitary fusion category $\mathcal{F}$, such that $K=K_{\mathcal{F}}$ (i.e. $K$ is hypergroup of the fusion ring of $\mathcal{F}$ ) and $\operatorname{Rep}\left(\mathcal{B}^{K}\right)$ is braided equivalent $Z(\mathcal{F})$.

Conversely, if $\mathcal{A}$ is a completely rational net with $\operatorname{Rep}(\mathcal{A})$ braided equivalent to the Drinfel'd center $Z(\mathcal{F})$ for a unitary fusion category $\mathcal{F}$, then there is a holomorphic net $\mathcal{B}$ and an action of the hypergroup $K_{\mathcal{F}}$ associated with $\mathcal{F}$, such that $\mathcal{B}^{K_{\mathcal{F}}}=\mathcal{A}$.

We note that in this case $\mathcal{B}^{K}$ is a quantum double net and $\mathcal{F}$ is a categorification of $K$. An interesting problem seems to be the following: Given a holomorphic net $\mathcal{B}$, find all finite index subnets $\mathcal{A}$. Then each subnet $\mathcal{A} \subset \mathcal{B}$ gives rise to a fusion category $\mathcal{F}_{\mathcal{A}}$.

We now want to discuss the case, where $\mathcal{B}$ is only assumed to be completely rational. Here one might ask: What are the possible representation categories of finite index subnets of a given net completely rational net $\mathcal{B}$ with known representation category?

Question 1.8. Let $\mathcal{B}$ be a completely rational net and $\mathcal{C}$ a unitary modular tensor category. Is there a finite index subnet $\mathcal{A} \subset \mathcal{B}$ with $\operatorname{Rep}(\mathcal{A})$ braided equivalent to $\mathcal{C}$ ?

In this case it is necessary that $\mathcal{C}$ and $\operatorname{Rep}(\mathcal{B})$ belong to the same Witt class [DMNO13. There is a more refined necessary - but not sufficient (see below) - condition for the existence of such a net $\mathcal{A}$ :

Proposition 1.9 (see Propopsition 5.11). Let $\mathcal{B}$ be a completely rational conformal net with $\mathcal{D}:=$ $\operatorname{Rep}(\mathcal{B})$. A necessary condition for the existence of a finite index subnet $\mathcal{A} \subset \mathcal{B}$ with $\operatorname{Rep}(\mathcal{A})$ braided equivalent to $\mathcal{C}$ is:

There is a fusion category $\mathcal{F}$ and an injective (full) central functor $\mathcal{D}^{\text {rev }} \rightarrow \mathcal{F}$, such that $\mathcal{C}$ is braided equivalent to the Müger centralizer $C_{Z(\mathcal{F})}\left(\mathcal{D}^{\text {rev }}\right)$ (also denoted by $\mathcal{D}^{\text {rev' }} \cap Z(\mathcal{F})$ ).

We note that this condition is not sufficient and stress the fact that the existence of subnets is not a purely categorical problem in the sense that it not only depends on $\operatorname{Rep}(\mathcal{B})$, but depends on the explicit net $\mathcal{B}$.

For example, take the moonshine net $\mathcal{A}^{\sharp}$ and $\mathcal{F} \cong \operatorname{Vect}_{G}$ for some finite group $G$ which does not embed into the monster group. Then there (trivially) exists such a braided central functor as above, but no action of $G$ on $\mathcal{A}^{\sharp}$ since $\operatorname{Aut}\left(\mathcal{A}^{\sharp}\right)$ is the monster group. A second family of examples are the Virasoro nets for $c<1$ [KL04] which are rational. The Virasoro net is minimal [Car98], so it has no proper subnets at all. But one can easily see that there is a $\mathbb{Z}_{2}$-simple current extension $\mathcal{B}_{k}$ of $\mathcal{A}_{\mathrm{SU}(2)_{k}} \otimes \mathcal{A}_{\mathrm{SU}(2)_{1}} \otimes \tilde{\mathcal{A}}_{k}$ fulfilling $\operatorname{Rep}\left(\mathcal{B}_{k}\right) \cong \operatorname{Rep}\left(\operatorname{Vir}_{c_{k}}\right)$ with $c_{k}=6-\frac{1}{(k+2)(k+3)}$. Here $\tilde{\mathcal{A}}_{k}$ is a net with $\operatorname{Rep}\left(\tilde{\mathcal{A}}_{k}\right) \cong \operatorname{Rep}\left(\mathcal{A}_{\mathrm{SU}(2)_{k}}\right)^{\text {rev }}$ constructed in $\mid$ Bis16b. But $\mathcal{B}_{k}$ has many non-trivial subnets.

We now state the general characterization result for proper finite hypergroup actions on a completely rational net $\mathcal{B}$. 
Theorem 1.10 (see Therorem 5.16). Let $\mathcal{B}$ be a completely rational conformal net with a proper action of a hypergroup $K$. Then there exists a unitary fusion category $\mathcal{F}$, such that $K$ is equivalent to $K_{\mathcal{F}} / / K_{\mathcal{B}}$, where $K_{\mathcal{B}}$ is the hypgergroup associated with $\operatorname{Rep}(\mathcal{B})$.

Furthermore, there is a central inclusion $\mathcal{G}:=\operatorname{Rep}(\mathcal{B})^{\mathrm{rev}} \subset \mathcal{F}$ and $\operatorname{Rep}\left(\mathcal{B}^{K}\right)$ is braided equivalent to $C_{Z(\mathcal{F})}(\mathcal{G})$, the Müger centralizer of $\mathcal{G}$ in $Z(\mathcal{F})$ (also denoted by $\mathcal{G}^{\prime} \cap Z(\mathcal{F})$ ).

While writing this manuscript, the author observed that a similar action of the double coset algebra was given in [Xu14, Section 2.11 and Theorem 3.8]. But there the focus was on intermediate nets in the case where a subnet is already known. A similar action on charged intertwiners arose in BKLR16, BKLR15 which was motivation for the present work. An action by stochastic maps on conformal nets seem to not have appeared in the literature before.

We get the following formulae for the index of an inclusion and the $\mu$-index:

$$
\left[\mathcal{B}(I): \mathcal{B}^{K / / H}(I)\right]=D(K / / H) \equiv \frac{D(K)}{D(H)}, \quad \quad \mu\left(\mathcal{B}^{K}\right)=\mu(\mathcal{B}) \cdot D(K)^{2} .
$$

We remember that the $\mu$-index $\mu(\mathcal{B})$ of a completely rational conformal net $\mathcal{B}$ coincides with the global dimension $\operatorname{Dim}(\operatorname{Rep}(\mathcal{B}))$ of its representation category.

An interesting problem seems to be: Let $\mathcal{B}$ be a diffeomorphism covariant completely rational net with central charge $c>1$, find all finite index subnets, or more general, find the lattice of all irreducible subnets $\mathcal{A} \subset \mathcal{B}$, or find all finite index subnets.

The structure of this article. In Section 2, we give some preliminaries on unitary fusion categories, hypergroups and conformal nets. In Section 3, we define generalized orbifolds in terms of actions of finite hypergroups and show that their fixed points give finite index subnets. In Section 4, we construct a hypergroup action from an arbitrary finite index inclusion of nets. We show that the action is unique and that if we start with a generalized orbifold of Section 3 , this reconstructs the original hypergroup and action. Intermediate nets correspond to subhypergroups and we get an action of the hypergroup of double cosets on the intermediate net. In Section 5 , we give characterization results on commutative Q-systems in unitary modular tensor categories. This gives categorical restrictions on possible inclusions of completely rational conformal nets and a complete characterization of actions of hypergroups on completely rational conformal nets and their generalized orbifolds. In Section 6, we give some outlook on possible generalization to infinite inclusions of conformal nets.

In Appendix A we collect some results on completely positive and stochastic maps. In Appendix $\mathrm{B}$, we state some results for tensor categories which are more general than the one for unitary fusion categories in the one in Section 5. Some of the results are implicitly in the literature or can derived from them.

Acknowledgements. The author likes to thank Luca Giorgetti, Yasuyuki Kawahigashi, Roberto Longo, Karl-Henning Rehren and Feng Xu for remarks on earlier versions of this manuscript and Masaki Izumi and Pinhas Grossmann for discussions. The results were improved and the manuscript completed while the author visited the Hausdorff Trimester Program "Von Neumann Algebras" and the author is grateful for the hospitality of the Hausdorff Research Institute for Mathematics (HIM) in Bonn.

\section{Prelimaries}

2.1. Unitary Fusion Categories. Let $M$ be a type III factor. We denote by $\operatorname{End}_{0}(M)$ the strict and rigid $\mathrm{C}^{*}$-tensor category of normal unital *-endomorphisms $\rho: M \rightarrow M$ with finite dimension $d \rho=[M: \rho(M)]^{\frac{1}{2}}$, where $[M: N]$ is the minimal Jones index. The tensor product is given by the composition $\rho \otimes \sigma=\rho \circ \sigma$ and morphisms are given by interwiners $\operatorname{Hom}(\rho, \sigma)=\{t \in M$ : 
$t \rho(m)=\sigma(m) t\}$. For $r \in \operatorname{Hom}(\rho, \tilde{\rho})$ and $s \in \operatorname{Hom}(\sigma, \tilde{\sigma})$ the tensor product of morphisms is given by $r \otimes s:=r \rho(s) \equiv \tilde{\rho}(s) r$. We denote $\langle\rho, \sigma\rangle=\operatorname{dim} \operatorname{Hom}(\rho, \sigma)$. For each $\rho \in \operatorname{End}_{0}(M)$ there is a conjugate $\bar{\rho}$ and a standard solution [LR97] of the conjugate equation. This gives an (essentially unique) spherical structure [LR97]. An object is called irreducible, if $\operatorname{Hom}(\rho, \rho)=\mathbb{C} \cdot 1$, which is exactly the case if $\rho(M) \subset M$ is irreducible, i.e. $\rho(M)^{\prime} \cap M=\mathbb{C} \cdot 1$. By a sector $[\rho]$ we denote the unitary equivalence class $\{\operatorname{Ad} u \circ \rho: u$ unitary in $M\}$ of an endomorphism $\rho$. There is a direct sum which well-defined on sectors, namely $[\rho] \oplus[\sigma]$ is given by the sector of $r_{1} \rho(\cdot) r_{1}^{*}+r_{2} \sigma(\cdot) r_{2}^{*}$ where $r_{i}^{*} r_{j}=\delta_{i j} 1$ and $r_{1} r_{1}^{*}+r_{2} r_{2}^{*}=1$ is a representation of the generators of the Cuntz algebra $\mathcal{O}_{2}$ in $M$. We refer to $\mid$ BKLR15] for more details.

Let $\mathcal{F}$ be a rigid $\mathrm{C}^{*}$-tensor category. Let $\rho \in \mathcal{F}$ be irreducible and $\sigma \in \mathcal{F}$ arbitrary. Then $\operatorname{Hom}(\rho, \sigma)$ is a Hilbert space with scalar product:

$$
(s, t)_{\operatorname{Hom}(\rho, \sigma)}=\Phi_{\rho}\left(s^{*} t\right) \quad \Longleftrightarrow \quad(s, t)_{\operatorname{Hom}(\rho, \sigma)} \cdot 1_{\rho}=s^{*} t,
$$

where $\Phi_{\rho}$ is the standard left inverse (see [LR97]) of $\rho$. Note that the first definition generalizes if $\rho$ is not irreducible, and the second if $\mathcal{F}$ is not rigid.

A unitary fusion category $\mathcal{F}$ is a semisimple rigid $\mathrm{C}^{*}$-tensor category with finitely many isomorphism classes of irreducible objects. It can always be realized (in an essentially unique way) as a full and replete subcategory of $\operatorname{End}(M)$, which is closed under direct sums and subobjects, where $M$ is the hyperfinite type $\mathrm{III}_{1}$ factor [Pop95, HY00| (see also [Izu15|).

This way $\mathcal{F} \subset \operatorname{End}_{0}(M)$ is completely specified by a choice of finitely many irreducible sectors $\operatorname{Irr}(\mathcal{F})$, such that

- $\left[\operatorname{id}_{M}\right] \in \operatorname{Irr}(\mathcal{F})$,

- $\operatorname{Irr}(\mathcal{F})$ is closed under fusion

$$
[\rho \circ \sigma]=\bigoplus_{[\tau] \in \operatorname{Irr}(\mathcal{F})} N_{\rho, \sigma}^{\tau}[\tau], \quad(\rho, \sigma \in \mathcal{F})
$$

for some non-negative integer coefficients $\left\{N_{\rho, \sigma}^{\tau}=\operatorname{dim} \operatorname{Hom}(\rho \otimes \sigma, \tau)\right\}_{[\rho],[\sigma],[\tau] \in \operatorname{Irr}(\mathcal{F})}$,

- $\operatorname{Irr}(\mathcal{F})$ is closed under conjugates/duals, i.e. if $[\rho] \in \operatorname{Irr}(\mathcal{F})$ then there is a conjugate $[\bar{\rho}] \in$ $\operatorname{Irr}(\mathcal{F})$.

The coefficients $\left\{N_{\rho, \sigma}^{\tau}\right\}$ are called the fusion coefficients. They are the structure constants of the associated fusion ring $\mathbb{Z} \operatorname{Irr}(\mathcal{F})$, see below.

The dimension function $[\rho] \mapsto d \rho$ coincides with the unique positive character $d: \operatorname{Irr}(\mathcal{F}) \rightarrow \mathbb{R}_{\geq 1}$ on the fusion ring, see Lemma 2.12. The complex vector space $\mathbb{C} \operatorname{Irr}(\mathcal{F})$ has a normalized trace $\operatorname{tr}([\rho])=\delta_{[\rho],\left[\mathrm{id}_{M}\right]}$ and is a finite dimensional $\mathrm{C}^{*}$-algebra and therefore isomorphic to a multi-matrix algebra $\bigoplus_{i} M_{d_{i}}(\mathbb{C})$.

Let $\mathcal{C}$ be a unitary braided fusion category, i.e. there is a natural family of unitaries $\{\varepsilon(\rho, \sigma) \in$ $\operatorname{Hom}(\rho, \sigma)\}$ fulfilling the usual definition of a braiding.

The Müger centralizer $C_{\mathcal{C}}(\mathcal{F})=\mathcal{F}^{\prime} \cap \mathcal{C}$ of a full subcategory $\mathcal{F}$ of a unitary braied fusion category $\mathcal{C}$ is defined to be the full subcategory

$$
C_{\mathcal{C}}(\mathcal{F})=\left\{\rho \in \mathcal{C}: \varepsilon(\rho, \sigma) \varepsilon(\sigma, \rho)=1_{\sigma \otimes \rho} \text { for all } \sigma \in \mathcal{F}\right\} .
$$

We call $\mathcal{C}$ a unitary modular tensor category (UMTC) if the Müger center $\mathcal{C}^{\prime} \cap \mathcal{C}$ is trivial, i.e. $C_{\mathcal{C}}(\mathcal{C}) \cong$ Vect. Further, if $\mathcal{D}$ is UMTC which is a full subcategory of a UMTC $\mathcal{C}$, then $\mathcal{C}$ is braided equivalent to $\mathcal{D} \otimes C_{\mathcal{C}}(\mathcal{D})$ by [Müg03c, Theorem 4.2].

2.2. Subfactors in Unitary Fusion Categories. We give a short background to subfactors related to a given unitary fusion category. We refer to [BKL15, BKLR15]. Let us assume that ${ }_{N} \mathcal{F}_{N} \subset \operatorname{End}(N)$ is a unitary fusion category. We can consider a subfactor $\iota(N) \subset M$, with finite index. Then there is a dual homorphism $\bar{\iota}: M \rightarrow N$. We from now on ask that an overfactor 
$N \subset M$ fulfills $\bar{\iota} \circ \iota \in{ }_{N} \mathcal{C}_{N}$. We get isometries $w: \operatorname{id}_{N} \rightarrow \bar{\iota} \circ \iota$ and $v: \operatorname{id}_{M} \rightarrow \iota \circ \bar{\iota}$ fulfilling the standard conjugate equation

$$
\begin{aligned}
& \left(1_{\bar{\iota}} \otimes v^{*}\right)\left(w \otimes 1_{\bar{\iota}}\right) \equiv \bar{\iota}\left(v^{*}\right) w=\lambda \cdot 1_{\bar{\iota}} \equiv \lambda \cdot 1_{N} \\
& \left(1_{\iota} \otimes w^{*}\right)\left(v \otimes 1_{\iota}\right) \equiv \iota\left(w^{*}\right) v=\lambda \cdot 1_{\iota} \equiv \lambda \cdot 1_{M}
\end{aligned}
$$

where $\lambda=[M: N]^{-\frac{1}{2}}$, where $[M: N]$ is the minimal index.

A triple $\Theta=(\theta, w, x)$ with $\theta \in \operatorname{End}(N)$ and isometries $w: \operatorname{id}_{N} \rightarrow \theta$ and $x: \theta \rightarrow \theta^{2}$, which we will graphically display as

$$
\sqrt[4]{d \theta} w=\varliminf_{w} \quad \sqrt[4]{d \theta} x=\underbrace{\theta}_{\theta}
$$

is called a Q-system (cf. Lon94, LR97]) if it fulfills

$$
\begin{aligned}
& x x=\theta(x) x \quad\left(x \otimes 1_{\theta}\right) x=\left(1_{\theta} \otimes x\right) x \\
& w^{*} x=\theta\left(w^{*}\right) x=\lambda 1_{\theta} \quad\left(w^{*} \otimes 1_{\theta}\right) x=\left(1_{\theta} \otimes w^{*}\right) x=\lambda 1_{\theta} \quad \text { (unit law) }
\end{aligned}
$$

where $\lambda=\sqrt{d \theta}^{-1}$. In graphical notation this reads:
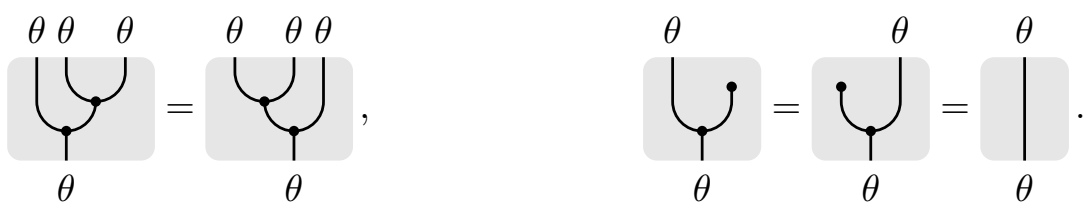

Overfactors $M \supset N$ with $\bar{\iota} \circ \iota \in{ }_{N} \mathcal{F}_{N}$ up to conjugation are in one-to-one correspondence with simple equivalence classes of Q-systems $(\theta, v, x)$ in ${ }_{N} \mathcal{F}_{N}$. The Q-system is given by $\theta=\bar{\iota} \circ \iota, w$ and $x:=\left(1_{\bar{\iota}} \otimes v \otimes 1_{\iota}\right)=\bar{\iota}(v) \in \operatorname{Hom}(\theta, \theta \circ \theta)$. The subfactor $N \subset M$ is called irreducible if $\iota(N)^{\prime} \cap M=\mathbb{C} \cdot 1_{M}$, which is equivalent with $\operatorname{dim} \operatorname{Hom}\left(\operatorname{id}_{N}, \theta\right)=1$. We call such a Q-system irreducible or connected. If not otherwise specified we mean by a Q-system a connected Q-system. We note that connected Q-systems are automatically simple. We denote by ${ }_{M} \mathcal{C}_{M}$ the unitary fusion category generated by $\beta \prec \iota \circ \rho \circ \bar{\iota}$. In this case we say, that ${ }_{N} \mathcal{C}_{N}$ an ${ }_{M} \mathcal{C}_{M}$ are Morita equivalent, which correspond to weak monoidal equivalence, see [Müg03a].

We are often interested in the case that ${ }_{N} \mathcal{C}_{N}$ is a UMTC and $N \subset M$ coming from a commutative Q-system in ${ }_{N} \mathcal{C}_{N}$. Then a Q-system is called commutative if $\varepsilon(\theta, \theta) x=x$, diagramatically:

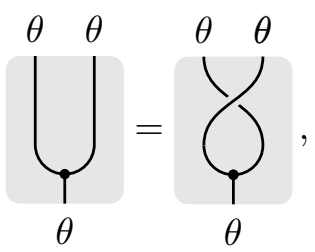

Let $\iota(N) \subset M$ associated to Q-system in ${ }_{N} \mathcal{C}_{N}$ For $\rho \in{ }_{N} \mathcal{C}_{N}$ we define its $\alpha$-induction by

$$
\alpha_{\rho}^{ \pm}=\bar{\iota}^{-1} \circ \operatorname{Ad}\left(\varepsilon^{ \pm}(\rho, \theta)\right) \circ \lambda \circ \bar{\iota} \in \operatorname{End}(M) .
$$

It turns out that $\alpha^{ \pm}:{ }_{N} \mathcal{C}_{N} \rightarrow{ }_{M} \mathcal{C}_{M}$. We denote by ${ }_{M} \mathcal{C}_{M}^{ \pm}=\left\langle\alpha^{+}(\rho): \rho \in{ }_{N} \mathcal{C}_{N}\right\}$ the unitary fusion category generated by $\alpha^{ \pm}$-induction, respectively. Because ${ }_{N} \mathcal{C}_{N}$ is a UMTC it follows that ${ }_{M} \mathcal{C}_{M}^{+} \cup{ }_{M} \mathcal{C}_{M}^{-}$generates ${ }_{M} \mathcal{C}_{M}$. We denote by ${ }_{M} \mathcal{C}_{M}^{0}={ }_{M} \mathcal{C}_{M}^{+} \cap{ }_{M} \mathcal{C}_{M}^{-}$the ambichiral category. 
2.3. The Drinfel'd center. The (unitary) Drinfel'd center or quantum double $Z\left({ }_{N} \mathcal{F}_{N}\right)$ is the category with objects $\left(\sigma, \varepsilon_{\sigma}\right)$, where $\sigma \in{ }_{N} \mathcal{F}_{N}$ and a (unitary) half-braiding $\varepsilon_{\sigma}=\left\{\varepsilon_{\sigma}(\rho)\right\}_{\rho \in{ }_{N} \mathcal{F}_{N}}$, i.e. a family of unitaries $\varepsilon_{\sigma}(\rho) \in \operatorname{Hom}(\sigma \rho, \rho \sigma)$, such that for every $t \in \operatorname{Hom}(\rho, \tau)$

$$
\left(t \otimes 1_{\sigma}\right) \cdot \varepsilon_{\sigma}(\rho)=\varepsilon_{\sigma}(\tau) \cdot\left(1_{\sigma} \otimes t\right)
$$

and

$$
\varepsilon_{\sigma}(\rho \tau)=\left(1_{\rho} \otimes \varepsilon_{\sigma}(\tau)\right) \cdot \varepsilon_{\sigma}(\rho) .
$$

We introduce the following intuitive graphical notation for half-braidings:

$$
\varepsilon_{\sigma}(\tau)=\gamma_{\sigma}^{\tau} \int_{\tau}^{\sigma} .
$$

The hooks at the end of the braiding symbolizes that the naturality in $\sigma$ does a priori not hold. Using this notations, the conditions on a half-brading reads as:
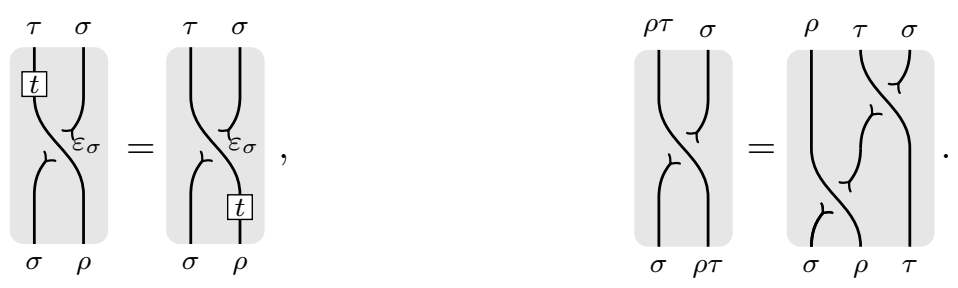

The morphisms are given by:

$$
\begin{aligned}
& \operatorname{Hom}\left(\left(\rho, \varepsilon_{\rho}\right),\left(\sigma, \varepsilon_{\sigma}\right)\right) \\
& =\left\{t \in \operatorname{Hom}(\rho, \sigma):\left(1_{\tau} \otimes t\right) \cdot \varepsilon_{\rho}(\tau)=\varepsilon_{\sigma}(\tau) \cdot\left(t \otimes 1_{\tau}\right) \text { for all } \tau \in{ }_{N} \mathcal{C}_{N}\right\} \\
& =\left\{t \in \operatorname{Hom}(\rho, \sigma):\left.\int_{\rho}^{\tau}\right|_{\tau} ^{\tau}=\left.\sum_{\rho}^{\tau}\right|_{\tau} ^{\sigma} \varepsilon_{\sigma} \text { for all } \tau \in{ }_{N} \mathcal{F}_{N}\right\} .
\end{aligned}
$$

and the tensor product by

$$
\begin{aligned}
& \left(\rho, \varepsilon_{\rho}\right) \otimes\left(\sigma, \varepsilon_{\sigma}\right)=\left(\rho \sigma,\left(\varepsilon_{\rho}(\cdot) \otimes 1_{\sigma}\right) \cdot\left(1_{\rho} \otimes \varepsilon_{\sigma}(\cdot)\right)\right), \\
& \left.\int_{\rho \sigma}^{\tau}\right|_{\tau} ^{\rho \sigma}=\left.\left.\right|_{\sigma} ^{\tau}\right|_{\tau} ^{\rho}
\end{aligned}
$$

The tensor product of morphisms is the usual one. Namely, it is easy to check that if $s \in$ $\operatorname{Hom}\left(\left(\sigma, \varepsilon_{\sigma}\right),\left(\sigma^{\prime}, \varepsilon_{\sigma^{\prime}}\right)\right)$ and $t \in \operatorname{Hom}\left(\left(\tau, \varepsilon_{\tau}\right),\left(\tau^{\prime}, \varepsilon_{\tau^{\prime}}\right)\right)$ then

$t \otimes s \in \operatorname{Hom}\left(\left(\sigma, \varepsilon_{\sigma}\right) \otimes\left(\tau, \varepsilon_{\tau}\right),\left(\sigma^{\prime}, \varepsilon_{\sigma^{\prime}}\right) \otimes\left(\tau^{\prime}, \varepsilon_{\tau^{\prime}}\right)\right) \equiv \operatorname{Hom}\left(\left(\sigma \tau, \varepsilon_{\sigma} \sigma\left(\varepsilon_{\tau}(\cdot)\right)\right),\left(\sigma^{\prime} \tau^{\prime}, \varepsilon_{\sigma^{\prime}} \sigma^{\prime}\left(\varepsilon_{\tau^{\prime}}(\cdot)\right)\right)\right)$. 
Note that this is again a strict tensor category and it is braided, with the braiding given as:

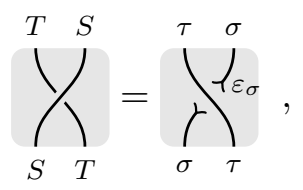

where $S=\left(\sigma, \varepsilon_{\sigma}\right)$ and $T=\left(\tau, \varepsilon_{\tau}\right)$.

2.4. Longo-Rehren Subfactors and Drinfel'd center. Let $\mathcal{F}$ be a unitary fusion category (UFC). We may assume that $\mathcal{F}={ }_{N} \mathcal{F}_{N} \subset \operatorname{End}(N)$ for $N$ a hyperfinite type $\mathrm{III}_{1}$ factor. For example, $\mathcal{F}$ might be the even part $\mathcal{F}=\langle\bar{\iota} \iota\rangle$ of a finite index, finite depth subfactor $\iota(N) \subset M$.

Starting from this data we can build the Longo-Rehren inclusion [LR95] as follows. Let $B=$ $N \otimes N^{\text {op }}$ and let $j: N \rightarrow N^{\text {op }}$ be an anti-linear isomorphism. For $\beta \in \operatorname{End}_{0}(N)$ we define $\beta^{\text {op }}=$ $j \circ \beta \circ j^{-1} \in \operatorname{End}_{0}\left(N^{\text {op }}\right)$. We denote by ${ }_{B} \mathcal{G}_{B}$ the unitary fusion category $\left\langle\rho \otimes \sigma^{\text {op }}: \rho, \sigma \in \mathcal{F}\right\rangle \subset$ $\operatorname{End}_{0}(B)$, see [LR95, Izu00]. This means, we have ${ }_{B} \mathcal{G}_{B} \cong \mathcal{F} \otimes \mathcal{F}^{\text {op }}$. There is a Q-system $(\gamma, v \in$ $\left.\operatorname{Hom}\left(\operatorname{id}_{B}, \gamma\right), z \in \operatorname{Hom}(\gamma, \gamma \gamma)\right)$ inside ${ }_{B} \mathcal{G}_{B}$ given by [LR95, Izu00]:

$$
\gamma=\sum \operatorname{Ad} v_{i} \circ\left(\rho_{i} \otimes \rho_{i}^{\mathrm{op}}\right), \quad v=v_{0}, \quad z=\frac{1}{\sqrt{\operatorname{Dim} \mathcal{F}}} \bigoplus_{i j k} \sum_{t \in \mathrm{B}\left(\rho_{i}, \rho_{j} \rho_{k}\right)} \sqrt{\frac{d \rho_{i} d \rho_{j}}{d \rho_{k}}} t \otimes j(t),
$$

where $\left\{v_{i}\right\}$ are generators of $\mathcal{O}_{n+1}$ and $\mathrm{B}\left(\rho_{i}, \rho_{j} \rho_{k}\right)$ is an orthonormal basis of $\operatorname{Hom}\left(\rho_{i}, \rho_{j} \rho_{k}\right)$. We get a subfactor $A=E(B) \subset B$, where $E(\cdot)=z^{*} \gamma(\cdot) z$ is the conditional expectation associated with $\Gamma$. We denote by $\iota: A \rightarrow B$ the canonical inclusion, then there is a dual $\bar{\iota}: B \rightarrow A$, such that $\gamma=\iota \bar{\iota}$, name $\bar{\iota}$ coincides with $\gamma$ seen as a map $B \rightarrow A$. Let ${ }_{A} \mathcal{G}_{A}$ be the unitary fusion category $\left\langle\bar{\iota}\left(\rho \otimes \sigma^{\mathrm{op}}\right) \iota: \rho, \sigma \in \mathcal{F}\right\rangle \subset \operatorname{End}_{0}(A)$. By [zu00, Müg03b] we get an equivalence of unitary fusion categories $\eta: Z(\mathcal{F}) \rightarrow{ }_{A} \mathcal{G}_{A}$, in particular ${ }_{A} \mathcal{G}_{A}$ has a non-degenerate braiding and therefore possesses the structure of a UMTC. The dual Q-system $\Theta=(\theta=\bar{\iota} \iota, w, x=\bar{\iota}(v))$ is a commutative Q-system in ${ }_{A} \mathcal{G}_{A}$. Since $(d \theta)^{2}=(d \gamma)^{2}=(\operatorname{Dim} \mathcal{F})^{2}=\operatorname{Dim}\left({ }_{A} \mathcal{G}_{A}\right)$ it is a Lagrangian Q-system, which is defined as follows.

Definition 2.1. We call an irreducible commutative Q-system $\Theta=(\theta, x, w)$ in a UMTC $\mathcal{C}$ Lagrangian if $(\operatorname{dim} \theta)^{2}=\operatorname{Dim} \mathcal{C}$.

We remark that $\left[\alpha_{\eta\left(\beta, \varepsilon_{\beta}\right)}^{+}\right]=[\beta \otimes \mathrm{id}]$ by $\operatorname{Izu00}$, Corollary 6.3].

Proposition 2.2. Let ${ }_{A} \mathcal{G}_{A} \cong Z(\mathcal{G})$ be the unitary modular tensor category from above. Then the category generated by $\alpha^{+}$-induction ${ }_{B} \mathcal{G}_{B}^{+}=\left\langle\alpha_{\rho}^{+}: \rho \in{ }_{A} \mathcal{G}_{A}\right\rangle$ coincides with $\langle\beta \otimes \mathrm{id}: \beta \in \mathcal{F}\rangle \cong \mathcal{F}$.

2.5. Hypergroups and Fusion Rings. We introduce the notion of a hypergroup. The reason is that we need a generalization of a fusion ring, where the coefficients are not necessarily integral. A fusion ring gives "up to a different normalization" a hypergroup. The converse is in general not true. The normalization of the hypergroup has a more probabilistic nature, while the fusion ring has more of a categorical or representation theoretical nature.

Definition 2.3. A (finite) hypergroup is a set $K=\left\{c_{0}, \ldots, c_{n}\right\}$ with an evolution $c_{i} \mapsto c_{\bar{i}}$ and a structure of an associative unital $*$-algebra structure on $\mathbb{C} K$ :

$$
c_{i} c_{j}=\sum_{k} C_{i j}^{k} c_{k}, \quad c_{k}^{*}=c_{\bar{k}}, \quad c_{0}^{*}=c_{0},
$$

with unit $c_{0}$, such that

(1) $C_{i j}^{k} \geq 0$

(2) $\sum_{k} C_{i j}^{k}=1$ for all $i, j$, and 
(3) $C_{i j}^{0}=C_{j i}^{0}$ and $C_{i j}^{0}>0$ iff $j=\bar{i}$.

The condition $C_{i j}^{0}=C_{j i}^{0}$ turns out to be automatic [SW03]. We note that $\mathbb{C} K:=\operatorname{span}_{\mathbb{C}}(K)$ is a $\mathrm{C}^{*}$-algebra with normalized trace defined by $\operatorname{tr}\left(c_{k}\right)=\delta_{k, 0}$.

Definition 2.4. A Haar element is an element $e \in \operatorname{Conv}(K)$, such that $e^{*}=e=e^{2}$ and $c_{k} e=e c_{k}=e$ for all $c_{k} \in K$.

We define the weight of an element $c_{i} \in K$ to be $w_{i}=\left(C_{i \bar{i}}^{0}\right)^{-1}$ and the weight $D(K)$ of the hypergroup $K$ to be $D(K)=\sum_{k} w_{k}$. Then it follows that $w_{i}=w_{\bar{i}}$.

Lemma 2.5. We have

$$
C_{i j}^{k}=\frac{w_{k}}{w_{i}} C_{k \bar{j}}^{i}=\frac{w_{k}}{w_{j}} C_{\bar{i} k}^{j}=\frac{w_{k}}{w_{i}} C_{j \bar{k}}^{\bar{i}} .
$$

Proof. The first equation follows from comparing the $c_{0}$ coefficients of $\left(c_{i} c_{j}\right) c_{\bar{k}}=w_{k}^{-1} C_{i j}^{k} c_{0}+\cdots$ and $\left(c_{i} c_{j} c_{\bar{k}}\right)^{*}=\left(c_{k} c_{\bar{j}}\right) c_{\bar{i}}=w_{i}^{-1} C_{k \bar{j}}^{i} c_{0}+\cdots$. The other equations are derived analogously.

Proposition 2.6. The convex sum

$$
e_{K}=\frac{1}{D} \sum_{c_{k} \in K} w_{k} c_{k}
$$

defines a Haar element on $K$.

Proof. Self-adjointness $e_{K}^{*}=e_{K}$ follows immediately. Further, we have:

$$
\begin{aligned}
e_{K} c_{\ell} & =\frac{1}{D} \sum_{k} w_{k} c_{k} c_{\ell}=\frac{1}{D} \sum_{k, m} w_{k} C_{k \ell}^{m} c_{m}=\frac{1}{D} \sum_{k, m} w_{m} C_{m \bar{\ell}}^{k} c_{m}=\frac{1}{D} \sum_{m} w_{m} c_{m}=e_{K}, \\
c_{\ell} e_{K} & =\frac{1}{D} \sum_{k} w_{k} c_{\ell} c_{k}=\frac{1}{D} \sum_{k, m} w_{k} C_{\ell k}^{m} c_{m}=\frac{1}{D} \sum_{k, m} w_{m} C_{m \bar{k}}^{\ell} c_{m}=\frac{1}{D} \sum_{m} w_{m}[m]=e_{K}, \\
e_{K}^{2} & =\frac{1}{D^{2}} \sum_{k, \ell} w_{k} w_{\ell} c_{k} c_{\ell}=\frac{1}{D^{2}} \sum_{k, \ell, m} w_{k} w_{\ell} C_{k \ell}^{m} c_{m}=\frac{1}{D^{2}} \sum_{k, \ell, m} w_{m} w_{\ell} C_{m \bar{\ell}}^{k} c_{m} \\
& =\frac{1}{D^{2}} \sum_{\ell, m} w_{m} w_{\ell} c_{m}=\frac{1}{D} \sum_{m} w_{m} w_{\ell} c_{m}=e_{K} .
\end{aligned}
$$

Example 2.7. Let $G$ be a finite group, then it is a hypergroup with $\bar{g}=g^{-1}$. In this case the Haar element is $e_{G}=\frac{1}{|G|} \sum_{g \in G} g$ and corresponds to the average with respect to the Haar measure.

Definition 2.8. A fusion ring (basis) is a set $F=\{[0], \ldots,[n]\}$ with an evolution $[i] \mapsto[\bar{i}]$ and a structure of an associative unital $*$-algebra structure on $\mathbb{C} F$ :

$$
[i][j]=\sum_{k} N_{i j}^{k}[k], \quad[i]^{*}=[\bar{i}],
$$

with [0] the unit, such that

(1) $N_{i j}^{k} \in \mathbb{Z}_{\geq 0}$, and

(2) $N_{i j}^{0}=\delta_{j, \bar{i}}$.

With this definition the ring $\mathbb{Z} F$ is a based ring [EGNO15, 3.1] which is a fusion ring. We drop the word basis if it is clear what we are talking about.

Lemma 2.9. Let $F$ be a fusion ring, then we have Frobenius reciprocity, i.e.

$$
N_{i j}^{k}=N_{k \bar{j}}^{i}=N_{\bar{i} k}^{j}=N_{j \bar{k}}^{\bar{i}}=N_{\bar{k} i}^{\bar{j}} .
$$


Proof. As in Lemma 2.5, or by noting that we get a hypergroup (see below).

Definition 2.10. If $\mathcal{F}$ is a unitary fusion category, then $F=\operatorname{Irr}(\mathcal{F})$ is a fusion ring with product

$$
[\rho] \otimes[\sigma]=\bigoplus_{\tau \in \operatorname{Irr}(\mathcal{F})} N_{\rho, \sigma}^{\tau}[\tau], \quad \quad N_{\rho, \sigma}^{\tau}=\operatorname{dim} \operatorname{Hom}(\rho \otimes \sigma, \tau)
$$

and $[\sigma]^{*}=[\bar{\sigma}]$. In this case we say $\mathcal{F}$ is a categorification of $F$. If a fusion ring $F$ has a categorification, we say it is categorifiable.

Usually, unitarity is not assumed, but in this paper we deal with operator algebras which naturally give unitary fusion categories. It is widely open problem which fusion rings are categorifiable. The following is a classical result.

Example 2.11. If $G$ is a finite group, then the categorifications are in one-to-one correspondence with elements in $H^{3}(G, \mathrm{U}(1))$ (see e.g. |FRS04|). We note that in operator algebraic terms, the categorifcation is given by $\left\langle\alpha_{g}: g \in G\right\rangle \subset \operatorname{End}(M)$, where $\alpha: G \rightarrow \operatorname{Out}(M)$ is a $G$-kernel Con77, Jon80 and $M$ a (hyperfinite) type $\mathrm{III}_{1}$ (or originally $\mathrm{II}_{1}$ ) factor.

The following is an application of Perron-Frobenius theory.

Lemma 2.12 ([Sun92, 10. Theorem]). There is a unique positive character $K \ni k \mapsto d_{k}$, such that $d_{0}=1$. It holds $d_{\bar{i}}=d_{i}$ and $d_{i} \geq 1$.

Proposition 2.13. A fusion ring basis $F$ is naturally a hypergroup $K_{F}$ by choosing $c_{i}=d_{i}^{-1}[i] \in$ $\mathbb{C} F$, and $K_{F}=\left\{c_{0}, \ldots, c_{n}\right\}$ with:

$$
C_{i j}^{k}=\frac{d_{k}}{d_{i} d_{j}} N_{i j}^{k}, \quad w_{i}=d_{i}^{2} .
$$

A hypergroup $K$ comes from a fusion ring if and only if:

$$
\sqrt{\frac{w_{i} w_{j}}{w_{k}}} C_{i j}^{k} \in \mathbb{Z}_{\geq 0} \quad \text { for all } i, j, k \in K .
$$

We define the analogous as for the hypergroup:

$$
D(F)=\sum_{k \in F} d_{k}^{2}, \quad \quad e_{F}=\frac{1}{D(F)} \sum_{k \in F} d_{k}[k] .
$$

Here $D(F)$ is called the global dimension of $F$ which coincides with the weight $D\left(K_{F}\right)$ of the associated hypergroup $K_{F}$. It follows that $e_{F}$ is the Haar element of the hypergroup. If $\mathcal{F}$ is a fusion category we denote the associated hypergroup by $K_{\mathcal{F}}$.

\subsection{Subhypergroups and Quotients.}

Definition 2.14. A subhypergroup $L \subset K$, written $L \leq K$ is a subset $L \subset K$, such that $\mathbb{C} L$ is a unital $*$-subalgebra of $\mathbb{C} K$, i.e. $c_{0} \in L, L L \subset \mathbb{C} L($ then $\mathbb{C} L L=\mathbb{C} L)$ and $L^{*}=L$.

For $x, y \in \operatorname{Conv}(K)$, we write $x \prec y$ if there is a $0<\lambda \leq 1$ and a $z \in \operatorname{Conv}(K)$, such that $y=\lambda x+(1-\lambda) z$. We write $\operatorname{supp}(x)=\left\{c_{k} \in K: c_{k} \prec x\right\}$.

Definition 2.15. Let $L, M$ be subhypergroups of $K$. We define the "set of $(L, M)$-double cosets" to be $L \backslash K / M=\left\{e_{L} c_{k} e_{M}: c_{k} \in K\right\}$, where $e_{L, M}$ is the Haar element associated with the corresponding subhypergroup. We write $K / / L=L \backslash K / L$.

The following is well-known see e.g. BH95 for the case of compact hypergroups.

Proposition 2.16. The double cosets $K / / L$ form a hypergroup. 
Proof. Since $e_{L}$ is a projection $\mathbb{C}[K / / L]=e_{L} \mathbb{C} K e_{L}$ is a $*$-algebra with identity $e_{L}$.

For $\left(e_{L} c_{k} e_{L}\right),\left(e_{L} c_{l} e_{L}\right) \in K / / L$, we have $\left(e_{L} c_{k} e_{L}\right)\left(e_{L} c_{l} e_{L}\right)=e_{L}\left(c_{k} e_{L} c_{l}\right) e_{L}=\sum_{[m]} \tilde{C}_{[k],[l]}^{[m]}\left(e_{L} c_{m} e_{L}\right)$ with $\tilde{C}_{[k],[l]}^{[m]} \geq 0$, where $[m]$ is the equivalence class $\left\{k: e_{L} c_{m} e_{L}=e_{L} c_{k} e_{L}\right\}$. Since $e_{L} c_{k} e_{L} \in \operatorname{Conv}(K)$ by applying the trivial representation $c_{k} \mapsto 1$, we get that $\sum_{[m]} \tilde{C}_{[k],[l]}^{[m]}=1$.

Since $e_{L} \prec e_{L} c_{k} c_{\bar{k}} \prec e_{L} c_{k} e_{L} c_{\bar{k}} e_{L}$, we have that $\left(e_{L} c_{k} e_{L}\right)\left(e_{L} c_{k} e_{L}\right)^{*}$ contains the identity. Conversely, let us assume that $e_{L} \prec e_{L} c_{k} e_{L} c_{l} e_{L}=\left(e_{L} c_{k} e_{L}\right)\left(e_{L} c_{l} e_{L}\right)$, then by [BH95, 1.5.14 Proposition] it follows that $e_{L} c_{k} e_{L}=e_{L} c_{l} e_{L}$.

A map $\phi: K \rightarrow L$ is a morphism if it extends to a $*$-homomorphism $\mathbb{C} K \rightarrow \mathbb{C} L$. We define $\operatorname{ker}(\phi)=\left\{c_{k} \in K: \phi\left(c_{k}\right)=c_{0}\right\}$ and $\operatorname{Im}(\phi)=\phi(K)$, which are subhypergroups of $K$ and $L$, respectively. With this notion we have a short exact sequence

$$
\left\{c_{0}\right\} \longrightarrow L \stackrel{\iota}{\longrightarrow} K \stackrel{e_{L} \cdot e_{L}}{\longrightarrow} K / / L \longrightarrow\left\{\tilde{c}_{0} \equiv e_{L}\right\} .
$$

Definition 2.17. $K=\bigcup_{g \in G} K_{g}$ is a grading of a hypergroup $K$ by a finite group $G$, if $c_{i} \in K_{g}$ and $c_{j} \in K_{h}$ implies $c_{i} c_{j} \in \operatorname{Conv}\left(K_{g h}\right)$. The grading is faithful if $K_{g}$ is non-empty for all $g \in G_{K}$.

If $K$ is graded by $G$, then $\mathbb{C} K=\bigoplus_{g \in G} \mathbb{C} K_{g}$ is a graded algebra. The adjoint subhypergroup $K_{\text {ad }}$ of $K$ is defined by $K_{\text {ad }}=\left\{c_{l} \in K: c_{l} \prec c_{k} c_{\bar{k}}\right.$ for some $\left.c_{k} \in K\right\}$. It follows that $G_{K}=K / / K_{\text {ad }}$ is a group, which we call the universal grading group of $K$ (cf. [EGNO15, Corollary 3.6.6]) and that $K_{g}=\left\{c_{k} \in K: e_{K_{\mathrm{ad}}} c_{k} e_{K_{\mathrm{ad}}}=g\right\}$ is a faithful grading with $K_{e}=K_{\mathrm{ad}}$. This grading is universal in the following sense: If $K=\bigcup_{g \in G} \tilde{K}_{g}$, is another faithful grading then there is a surjective group homomorphism $a: G_{K} \rightarrow G$ with $\tilde{K}_{g}=\bigcup_{h \in a^{-1}(g)} K_{h}$. The proofs are the same as for based rings, see e.g. EGNO15, Section 3.6]. Note, if $K$ is actually a group, then $G_{\text {ad }}=\left\{c_{0}\right\}$ and $G_{K}=K$. Further, $K$ has a non-trivial faithful gradings if and only if $K_{\mathrm{ad}} \neq K$.

2.7. Conformal Nets. We denote by Möb the group of Möbius transformations of the circle $\mathbb{S}^{1}$, which can be identified with $\mathrm{PSU}(1,1)$. By a conformal net $\mathcal{A}$ we mean a local Möbius covariant net on the circle, i.e. a map $\mathcal{I} \ni I \mapsto \mathcal{A}(I) \subset \mathcal{B}(\mathcal{H})$ from the set $\mathcal{I}$ of proper intervals $I \subset \mathbb{S}^{1} \subset \mathbb{C}$ on the circle to von Neumann algebras on a fixed separable Hilbert space $\mathcal{H}=\mathcal{H}_{\mathcal{A}}$, such that the following properties hold:

A. Isotony. $I_{1} \subset I_{2}$ implies $\mathcal{A}\left(I_{1}\right) \subset \mathcal{A}\left(I_{2}\right)$.

B. Locality. $I_{1} \cap I_{2}=\emptyset$ implies $\left[\mathcal{A}\left(I_{1}\right), \mathcal{A}\left(I_{2}\right)\right]=\{0\}$.

C. Möbius covariance. There is a strongly continuous unitary representation $U$ of Möb on $\mathcal{H}$, such that $U(g) \mathcal{A}(I) U(g)^{*}=\mathcal{A}(g I)$.

D. Positivity of energy. $U$ is a positive energy representation, i.e. the generator $L_{0}$ (conformal Hamiltonian) of the rotation subgroup $U\left(z \mapsto \mathrm{e}^{\mathrm{i} \theta} z\right)=\mathrm{e}^{\mathrm{i} \theta L_{0}}$ has positive spectrum.

E. Vacuum. There is a (up to phase) unique rotation invariant unit vector $\Omega \in \mathcal{H}$, which is cyclic for the von Neumann algebra $\mathcal{A}:=\bigvee_{I \in \mathcal{I}} \mathcal{A}(I)$.

By the Reeh-Schlieder property [FJ96] the vector $\Omega$ is cyclic and separating for every $\mathcal{A}(I)$. The Bisognano-Wichmann property holds GF93. BGL93. It states that for every $I \in \mathcal{I}$, there is a one-parameter subgroup $\left\{\delta_{I}(t)\right\}_{t \in \mathbb{R}} \subset$ Möb which fixes the endpoints of $I$, such that

$$
\Delta_{(\mathcal{A}(I), \Omega)}=U\left(\delta_{I}(-2 \pi t)\right), \quad J=U\left(r_{I}\right),
$$

are the Tomita-Takesaki modular objects associated with $(\mathcal{A}(I), \Omega)$. Here $U\left(r_{I}\right)$ is an anti-unitary representing the reflection along the interval $I$ which extends $U$ to an (anti-) unitary representation of Möb $\rtimes \mathbb{Z}_{2}$. This implies Haag-duality, i.e. $\mathcal{A}(I)^{\prime}=\mathcal{A}\left(I^{\prime}\right)$ for all $I \in \mathcal{I}$, where $I^{\prime}=\mathbb{S}^{1} \backslash \bar{I}$. The uniqueness of the vacuum implies that $\mathcal{A}(I)$ is either $\mathbb{C}$ or a type $\mathrm{III}_{1}$ factor in Connes classification Con73.

A local Möbius covariant net on $\mathcal{A}$ on $\mathbb{S}^{1}$ is called completely rational if it 
F. fulfills the split property, i.e. for $I_{0}, I \in \mathcal{I}$ with $\overline{I_{0}} \subset I$ the inclusion $\mathcal{A}\left(I_{0}\right) \subset \mathcal{A}(I)$ is a split inclusion, namely there exists an intermediate type I factor $M$, such that $\mathcal{A}\left(I_{0}\right) \subset M \subset \mathcal{A}(I)$.

G. is strongly additive, i.e. for $I_{1}, I_{2} \in \mathcal{I}$ two adjacent intervals obtained by removing a single point from an interval $I \in \mathcal{I}$ the equality $\mathcal{A}\left(I_{1}\right) \vee \mathcal{A}\left(I_{2}\right)=\mathcal{A}(I)$ holds.

H. for $I_{1}, I_{3} \in \mathcal{I}$ two intervals with disjoint closure and $I_{2}, I_{4} \in \mathcal{I}$ the two components of $\left(I_{1} \cup I_{3}\right)^{\prime}$, the $\mu$-index of $\mathcal{A}$

$$
\mu(\mathcal{A}):=\left[\left(\mathcal{A}\left(I_{2}\right) \vee \mathcal{A}\left(I_{4}\right)\right)^{\prime}: \mathcal{A}\left(I_{1}\right) \vee \mathcal{A}\left(I_{3}\right)\right]
$$

(which does not depend on the intervals $I_{i}$ ) is finite.

The split property implies that each $\mathcal{A}(I)$ is isomorphic to the unique (by [Haa87]) hyperfinite type $\mathrm{III}_{1}$ factor.

A representation $\pi$ of $\mathcal{A}$ is a family of representations $\pi=\left\{\pi_{I}: \mathcal{A}(I) \rightarrow \mathrm{B}\left(\mathcal{H}_{\pi}\right)\right\}_{I \in \mathcal{I}}$ on a separable Hilbert space $\mathcal{H}_{\pi}$. The family is asked to be compatible, i.e. $\pi_{J}\left\lceil\mathcal{A}(I)=\pi_{I}\right.$ for $I \subset J$. Every representation $\pi$ turns out (for any choice of an interval $I_{0} \in \mathcal{I}$ ) to be equivalent to a representation $\rho$ on $\mathcal{H}$ which is "localized in $I_{0}$ ", i.e. $\rho_{J}=\operatorname{id}_{\mathcal{A}(J)}$ for $J \cap I_{0}=\emptyset$. Then Haag duality implies that $\rho_{I}$ is an endomorphism of $\mathcal{A}(I)$ for every $I \in \mathcal{I}$ with $I \supset I_{0}$. The statistical dimension of $\rho \in \operatorname{Rep}^{I}(\mathcal{A})$ is given by $d \rho=[\mathcal{A}(I): \rho(\mathcal{A}(I))]^{\frac{1}{2}}$. Thus we can realize the category of in $I$ localized representations of $\mathcal{A}$ with finite statistical dimension inside the $\mathrm{C}^{*}$-tensor category of endomorphisms $\operatorname{End}_{0}(A)$ of a type III factor $A=\mathcal{A}(I)$ and the embedding turns out to be full and replete. We denote this category by $\operatorname{Rep}^{I}(\mathcal{A})$. In particular, this gives the representations of $\mathcal{A}$ the structure of a tensor category [DHR71]. It has a natural braiding, which is completely fixed by asking that if $\rho$ is localized in $I_{1}$ and $\sigma$ in $I_{2}$ where $I_{1}$ is left of $I_{2}$ inside $I$ then $\varepsilon(\rho, \sigma)=1$ [FRS89]. Let $\mathcal{A}$ be a completely rational conformal net, then by [KLM01] every representation is reducible and every irreducible representation has finite statistical dimension. Again by $|\operatorname{KLM} 01| \operatorname{Rep}^{I}(\mathcal{A})$ is a UMTC and the $\mu$-index $\mu_{\mathcal{A}}$ coincides with the global dimension $\operatorname{Dim}\left(\operatorname{Rep}^{I}(\mathcal{A})\right)$. We note that if $\mathcal{A}$ is strongly additive than every representation with finite statistical dimension is covariant [GL92].

Given a net $\mathcal{B}$ and an assignment $\mathcal{I} \ni I \mapsto \mathcal{A}(I) \subset \mathcal{B}(I)$ for all $I \subset \mathcal{I}$, which satisfies isotony and such that $U(g) \mathcal{A}(I) U(g)^{*}=\mathcal{A}(g I)$ for all $I \in \mathcal{I}$ and $g \in$ Möb is called a subnet $\mathcal{A}$ of $\mathcal{B}$. Let $e$ be the projection onto $\overline{\mathcal{A}(I) \Omega}$ which by the Reeh-Schlieder property is independent of $I$, then $e \mathcal{A}(I)$ is a conformal net on $e \mathcal{H}$, which by abuse of notation we also denote by $\mathcal{A}$. We write $\mathcal{A} \subset \mathcal{B}$. More general, given two independent conformal nets $\mathcal{A}$ and $\mathcal{B}$ we write $\mathcal{A} \subset \mathcal{B}$ or $\mathcal{B} \supset \mathcal{A}$ if there is a representation $\pi=\left\{\pi_{I}: \mathcal{A}(I) \rightarrow \mathcal{B}(I) \subset \mathrm{B}\left(\mathcal{H}_{\mathcal{B}}\right)\right\}$ of $\mathcal{A}$ on $\mathcal{H}_{\mathcal{B}}$ and an isometry $V: \mathcal{H}_{\mathcal{A}} \rightarrow \mathcal{H}_{\mathcal{B}}$ with $V \Omega_{\mathcal{A}}=\Omega_{\mathcal{B}}$ and $V U_{\mathcal{A}}(g)=U_{\mathcal{B}}(g) V$ and $V a=\pi_{I}(a) V$ for $I \in \mathcal{I}, a \in \mathcal{A}(I)$. Define $p$ the projection onto $\mathcal{H}_{\mathcal{A}_{0}}=\overline{\pi_{I}(\mathcal{A}(I)) \Omega}$. Then $p V$ is a unitary equivalence of the nets $\mathcal{A}$ on $\mathcal{H}_{\mathcal{A}}$ and $\mathcal{A}_{0}$ defined by $\mathcal{A}_{0}(I)=\pi_{I}(\mathcal{A}(I)) p$ on $\mathcal{H}_{\mathcal{A}_{0}}$. Here $\mathcal{A}_{0}$ is a subnet of $\mathcal{B}$ in the sense above.

\section{Hypergroup Actions on Conformal Nets}

We define actions of a hypergroup on a von Neumann algebras. Having the applications of actions on a conformal net in mind, we just concentrate on a very special case.

3.1. Hypergroup actions on non-commutative probability spaces. Given $(M, \Omega)$ a von Neumann algebra $M \subset \mathrm{B}(\mathcal{H})$ with a cyclic and separating vector $\Omega \in \mathcal{H}$ let us denote by $\operatorname{Stoch}_{\Omega}(M)$ the set of all $\Omega$-preserving stochastic maps $M \rightarrow M$, i.e. normal unital completely positive maps $\phi: M \rightarrow M$, such that $(\Omega, \phi(\cdot) \Omega)=(\Omega, \cdot \Omega)$, see Appendix A. We say a map $\phi \in \operatorname{Stoch}_{\Omega}(M)$ is extremal or pure if it cannot be written as a non-trivial convex combination of stochastic maps, i.e. $\phi=\lambda \phi_{1}+(1-\lambda) \phi_{2}$ with $\phi_{1}, \phi_{2} \in \operatorname{Stoch}_{\Omega}(M)$ and $\lambda \in(0,1)$ implies $\phi_{1}=\phi_{2}=\phi$.

Definition 3.1. Let $K$ be a hypergroup with structure constants $\left(C_{i j}^{k}\right)_{i, j, k \in K}$. A (normal) $\Omega$ preserving action of $K$ on $(M, \Omega)$ is a map $\phi: K \rightarrow \operatorname{Stoch}_{\Omega}(M), k \mapsto \phi_{k}$, s.t. for all $i, j \in K$ :

(1) $\phi_{i} \circ \phi_{j}=\sum_{k \in K} C_{i j}^{k} \phi_{k}$ and $\phi_{0}=\operatorname{id}_{M}$. 
(2) $\phi_{\bar{i}}$ is an $\Omega$-adjoint $\phi_{i}^{\sharp}$ of $\phi_{\bar{i}}$.

(3) $\phi_{i}$ is extremal in $\operatorname{Stoch}_{\Omega}(M)$

We say it is faithful if $\left\{\phi_{k}\right\}_{0 \leq k \leq n}$ are affine independent, i.e. $\left\{\phi_{k}-\mathrm{id}_{M}\right\}_{1 \leq k \leq 0}$ are linearly independent.

Proposition 3.2. Let $\phi: K \rightarrow \operatorname{Stoch}_{\Omega}(M)$ be a (normal) $\Omega$-preserving action of $K$ on $(M, \Omega)$. Then the following are equivalent.

(1) The action $\phi$ is faithful and

(2) $\phi_{k}=\phi_{0}$ implies $k=0$.

Proof. If $\phi_{k}=\phi_{0}$ for some $k \neq 0$, then the action is not faithful. Conversely, let us assume that the action is not faithful. i.e. there is a convex combination $\sum \lambda_{i=1}^{n} \phi_{i}=\phi_{0}$ with $\lambda_{k}>0$ for some $k$. Then $\phi_{\bar{k}}=\phi_{\bar{k}}\left(\sum \lambda_{i=1}^{n} \phi_{i}\right)=\lambda_{k} w_{k} \phi_{0}+\cdots$ and because $\phi_{\bar{k}}$ is pure it follows that $\phi_{\bar{k}}=\phi_{0}$.

We can extend $\phi$ to an affine map $\phi: \operatorname{Conv}(K) \rightarrow \operatorname{Stoch}_{\Omega}(M)$ and a linear map $\phi: \mathbb{C} K \rightarrow$ $\operatorname{Maps}(M)$ by $\phi\left(\sum_{k} \lambda_{k} c_{k}\right)=\lambda_{k} \phi_{k}$. From the Haar element $e_{K} \in \operatorname{Conv}(K)$ we get a $\Omega$-preserving stochastic map which is an idempotent

$$
E=\phi\left(e_{K}\right) \equiv \frac{1}{D(K)} \sum_{k \in K} w_{k} \phi_{k} .
$$

For $k \in K$ we define operators $V_{k} \in \mathrm{B}(\mathcal{H})$ by

$$
V_{k}: a \Omega \mapsto \phi_{k}(a) \Omega, \quad(a \in M)
$$

which are contractions by the Kadison-Schwarz inequality (5). This gives a representation of $K$ which can linearly be extended to a $*$-representation $V: \mathbb{C} K \rightarrow \mathrm{B}(\mathcal{H})$, namely

$$
\left(a \Omega, V_{\bar{k}} b \Omega\right)=\left(a \Omega, \phi_{\bar{k}}(b) \Omega\right)=\left(a \Omega, \phi_{k}^{\sharp}(b) \Omega\right)=\left(\phi_{k}(a) \Omega, b \Omega\right)=\left(V_{k} a \Omega, b \Omega\right)=\left(a \Omega, V_{k}^{*} b \Omega\right) .
$$

Proposition 3.3. The following subsets of $M$ are equal:

(1) $\left\{m \in M: \phi_{k}(m)=m\right.$ for all $\left.k \in K\right\}$,

(2) $E(M)$

(3) $M \cap\{e\}^{\prime}$,

(4) $M \cap V(\mathbb{C} K)^{\prime}$.

They give a unital von Neumman subalgebra $N \subset M$ with normal conditional expectation $E: M \rightarrow$ $N \subset M$.

Proof. $N=E(M)$ is a von Neumann algebra with conditional expectation $E: M \rightarrow N \subset M$ by Proposition A.3.

The inclusion $\left\{m \in M: \phi_{k}(m)=m\right.$ for all $\left.k \in K\right\} \subset E(M)$ is trivial. Let $m \in E(M)$. Because $E$ is an idempotent $m=E(m)$ and therefore $\phi_{k}(m)=\phi_{k} \circ E(m)=E(m)=m$. Since $\Omega$ is separating $M \ni m \mapsto m e$ is injective and $e x e=E(x) e$ which implies that: $m \in M$ commutes with $e$ if and only if $E(m)=m$ (cf. [JS97]).

We have $M \cap V(\mathbb{C} K)^{\prime} \subset M \cap\{e\}^{\prime}$, since $e=V\left(e_{K}\right)$. It remains to show $N:=\left\{m \in M: \phi_{k}(m)=\right.$ $m$ for all $k \in K\} \subset M \cap V(\mathbb{C} K)^{\prime}$, i.e. that $N \subset V(\mathbb{C} K)^{\prime}$. Let $n \in N$. Since $E$ is a conditional expectation onto $N$ we have $E\left(n^{*} n\right)=n^{*} n$. Then

$$
\phi_{k}\left(n^{*} n\right)=\phi_{k}\left(E\left(n^{*} n\right)\right)=E\left(n^{*} n\right)=n^{*} n=\phi_{k}(n)^{*} \phi_{k}(n) .
$$

For all $m \in M$ we have $\phi_{k}(n m)=\phi_{k}(n) \phi_{k}(m)=n \phi_{k}(m)$ using Theorem A.1 and therefore

$$
V_{k} n m \Omega=\phi_{k}(n m) \Omega=n \phi_{k}(m) \Omega=n V_{k} m \Omega .
$$

Since $\Omega$ is cyclic for $M$ this gives $V_{k} n=n V_{k}$ and since $k$ was arbitrary we have $n \in V(\mathbb{C} K)^{\prime}$. 
We denote the set of Proposition 3.3 by $M^{K}$ if it is clear which action $K \rightarrow \operatorname{Stoch}_{\Omega}(M)$ is meant and call $M^{K}$ the $K$-fixed point algebra. In particular, it follows that $e=V\left(e_{K}\right)$ is the Jones projection implementing the conditional expectation $E$, i.e. $E(\cdot) e=e E(\cdot) e$. In a similar fashion, $V_{k}$ is "implementing" $\phi_{k}$ :

Proposition 3.4. We have $\phi_{k}\left(n_{1} m n_{2}\right)=n_{1} \phi_{k}(m) n_{2}$ for all $n_{1}, n_{2} \in M^{K}$ and $m \in M$ and $\phi_{k}(m) e=V_{k} m e$ for all $m \in M$.

Proof. Using Theorem A.1 as before we get the first statement. For all $m_{1}, m_{2} \in M$ we have:

$$
\phi_{k}\left(m_{1}\right) e m_{2} \Omega=\phi_{k}\left(m_{1}\right) E\left(m_{2}\right) \Omega=\phi_{k}\left(m_{1} E\left(m_{2}\right)\right) \Omega=V_{k} m_{1} E\left(m_{2}\right) \Omega=V_{k} m_{1} e m_{2} \Omega
$$

and the statement follows because $\Omega$ is cyclic for $M$.

Remark 3.5. We can recover $\phi_{k}$ from $V_{k}$ as follows. Since $\left\{m^{\prime} \Omega: m^{\prime} \in M^{\prime}\right\}$ is dense in $\mathcal{H}$, we have that $\phi_{k}(m)$ with $m \in M$ is the closure of the linear map

$$
m^{\prime} \Omega \mapsto m^{\prime} V_{k} m \Omega, \quad m^{\prime} \in M^{\prime} .
$$

In our application, we have that the centralizer $M^{\varphi}=\mathbb{C} \cdot 1$ for the state $\varphi=(\Omega, \cdot \Omega)$ is trivial. This implies that $M^{K}$ is a factor by the following simple lemma.

Lemma 3.6. Let $M$ be a von Neumann algebra, $\varphi$ a state on $M$ and $E: M \rightarrow M$ a state preserving conditional expectation onto $N$, then $N^{\prime} \cap N \subset M^{\varphi}=\{m \in M: \varphi(n m)=\varphi(n m)$ for all $n \in N\}$.

Proof. Let $n \in N^{\prime} \cap N$. Then for every $m \in M$ we have $E(n m)=n E(m)=E(m) n=E(m n)$ and thus $\varphi(m n)=\varphi \circ E(m n)=\varphi \circ E(n m)=\varphi(n m)$ thus $n \in M^{\varphi}$.

We note that we get that the index is finite with $[M: N] \leq D$. Namely, we have the PimsnerPopa bound:

$$
E\left(m_{+}\right)=\frac{1}{D} \sum_{k} d_{k} \phi_{k}\left(m_{+}\right) \geq \frac{1}{D} \phi_{0}\left(m_{+}\right)=\frac{1}{D} m_{+}, \quad m_{+} \in M_{+} .
$$

If we have that $N$ is purely infinite factor, then $[M: N]=D$. Namely, in this case we know that the minimal index $[M: N] \equiv \inf \left\{c \in \mathbb{R}: c \cdot E-\operatorname{id}_{M}\right.$ is positive $\}$ coincides with $\inf \{c \in \mathbb{R}$ : $c \cdot E-\operatorname{id}_{M}$ is completely positive $\}$ by [BDH88, Was90]. If we asssume $[M: N]<D$, then there is an $\epsilon>0$, such that $E-\left(\frac{1}{D}+\epsilon\right) \operatorname{id}_{M}=\sum_{k \neq 0} \frac{w_{k}}{D} \phi_{k}-\epsilon \operatorname{id}_{M}$ is completely positive. This means $\left(1-\epsilon-D^{-1}\right)^{-1}\left(\sum_{k \neq 0} \frac{w_{k}}{D} \phi_{k}-\epsilon \operatorname{id}_{M}\right) \in \operatorname{Conv}\left(\left\{\phi_{0}, \ldots, \phi_{k}\right\}\right)$, which is a contradiction, since the $\left\{\phi_{0}, \ldots, \phi_{k}\right\}$ are affine independent.

\subsection{Hypergroup Action on Nets.}

Definition 3.7. Let $K$ be a hypergroup. A proper hypergroup action of $K$ on a conformal net $\mathcal{B}$ is a family $\left\{\phi^{I}: K \rightarrow \operatorname{Stoch}_{\Omega}(\mathcal{B}(I))\right\}_{I \in \mathcal{I}}$ of faithful $\Omega$-preserving normal hypergroup actions, which is compatible, i.e. $\phi_{k}^{J} \uparrow \mathcal{B}(I)=\phi_{k}^{I}$ for all $k \in K$ and all $I, J \in \mathcal{I}$ with $I \subset J$.

We observe that a proper hypergroup action is by definition vacuum preserving, i.e. $\varphi \circ \phi_{k}^{I}=\varphi$ for all $k \in K$ and all $I \in \mathcal{I}$. Here $\varphi(\cdot)=(\Omega, \cdot \Omega)$ is the vacuum state.

Let $K$ be a proper hypergroup action on $\mathcal{B}$. As before, we define $V_{k} \in \mathrm{B}(\mathcal{H})$ by

$$
V_{k}: a \Omega \mapsto \phi_{k}^{I}(a) \Omega, \quad a \in \mathcal{B}(I)
$$

and note that this is independent of $I \in \mathcal{I}$. This gives a representation of $K$ which extends to a $*-$ representation of $\mathbb{C} K$ on $\mathcal{H}$. Further we get conditional expectations $E_{I}=\phi^{I}\left(e_{K}\right)$. The conditional expectations are implemented by the Jones projection $e=V\left(e_{K}\right)$. We get that the local algebras have trivial centralizers $\mathcal{B}(I)^{\varphi}=\mathbb{C} \cdot 1$ for every $I \in \mathcal{I}$ by using covariance and positivity of energy and Lon79, Proof of Theorem 3]. Therefore by Lemma 3.6 it follows that the subalgebra $\mathcal{B}(I)^{K} \subset \mathcal{B}(I)$ is a subfactor. It turns out that $\left\{\mathcal{B}(I)^{K} \subset \mathcal{B}(I)\right\}_{I \in \mathcal{I}}$ is indeed a conformal subnet. 
Theorem 3.8. Let $\mathcal{B}$ be a conformal net and let $K$ be a hypergroup acting properly on $\mathcal{B}$.

(1) Then $I \mapsto \mathcal{B}(I)^{K}$ is a subnet of $\mathcal{B}$. In particular, $\mathcal{B}^{K}$ defined by $\mathcal{B}^{K}(I):=e \mathcal{B}(I)^{K}$ is a

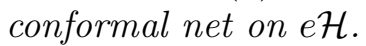

(2) The index is finite and given by $\left[\mathcal{B}(I): \mathcal{B}(I)^{K}\right]=D(K)$.

(3) If $\mathcal{B}$ is split and strongly additive, then $\mathcal{B}^{K}$ is split and strongly additive.

(4) If $\mathcal{B}$ is completely rational, then $\mathcal{B}^{K}$ is completely rational.

Proof. Define $\mathcal{A}(I)=\mathcal{B}(I)^{K}$, then $\left\{E_{I}: \mathcal{B}(I) \rightarrow \mathcal{A}(I) \subset \mathcal{B}(I)\right\}$ is a compatible family of vacuum preserving conditional expectations and thus the statement follows from the following Lemma 3.9.

First we observe that $\left[\mathcal{B}(I): \mathcal{B}(I)^{K}\right] \leq D(K)$. But then $\left(\mathcal{B}(I)^{K}\right)^{\prime} \cap \mathcal{B}(I)=\mathbb{C}$ by Lon03, Lemma 14] and $\mathcal{B}(I)^{K}$ is a factor and therefore $\left[\mathcal{B}(I): \mathcal{B}(I)^{K}\right]=D(K)$. The last two statements follow from [Lon03, Proposition 34, Theorem 24].

Lemma 3.9. Let $E_{I}: \mathcal{B}(I) \rightarrow \mathcal{A}(I) \subset \mathcal{B}(I)$ be a family of normal compatible and vacuum preserving conditional expectations with image $\mathcal{A}(I)=E_{I}(\mathcal{B}(I))$. Then $\mathcal{A}(I) \subset \mathcal{B}(I)$ is a conformal subnet and in particular the projection e onto $\overline{\mathcal{A}(I) \Omega}$ commutes with the Möbius action $U(g)$.

Proof. The Jones projection $e=e_{I}$ onto $\overline{\mathcal{A}(I) \Omega}$ does not depend $I \in \mathcal{I}$. Then $\mathcal{A}(I)=\mathcal{B}(I) \cap\{e\}^{\prime}$. Since $E_{I}$ preserves $(\Omega, \cdot \Omega)$ by Takesaki's theorem [Tak72], [Tak03, Theorem IX.4.2.] we have that $\operatorname{Ad} \Delta_{(\mathcal{B}(I), \Omega)}^{\mathrm{it}}$ leaves $\mathcal{A}(I)$ globally invariant, which is equivalent to $\left[\Delta_{(\mathcal{B}(I), \Omega)}^{\mathrm{i} t}, e\right]=0$. Thus by the Bisognano-Wichmann property it follows that $e$ commutes with the dilation $U\left(\delta_{I}(t)\right)$ of every interval $I \in \mathcal{I}$. But these generate the Möbius group, thus $[e, U(\cdot)]=0$ and $U(g) \mathcal{A}(I) U(g)^{*}=$ $U(g) \mathcal{B}(I) U(g)^{*} \cap\{e\}^{\prime}=\mathcal{B}(g I) \cap\{e\}^{\prime}=\mathcal{A}(g I)$. Isotony of $\mathcal{A}$ follows from the compatibility, thus $\mathcal{A} \subset \mathcal{B}$ is a conformal subnet.

\section{Hypergroup Actions from Subfactors and Conformal Subnets}

Let $\mathcal{A} \subset \mathcal{B}$ be a finite index subnet and let $A=\mathcal{A}(I)$ and $B=\mathcal{B}(I)$ for some fixed $I \in \mathcal{I}$. The inclusion is automatically irreducible [Lon03, Lemma 14], i.e. $\mathcal{A}(I)^{\prime} \cap \mathcal{B}(I)=\mathbb{C}$ for all $I \in \mathcal{I}$. By the Bisognano-Wichmann property and Takesaki's theorem there is a unique ([Lon89, p. 230]) normal conditional expectation $E: B \rightarrow A$ which is implemented by the projection $e_{A}$ onto $\overline{A \Omega}$. By the Reeh-Schlieder property $\mid$ FJ96 $\mid \overline{\mathcal{A}(I) \Omega}=\overline{\mathcal{A} \Omega}$ and in particular the Jones projection $e_{\mathcal{A}}:=e_{A}$ does not depend on $I$. In particular, there is a unique family of conditional expectations $\left\{E_{I}: \mathcal{B}(I) \rightarrow\right.$ $\mathcal{A}(I) \subset \mathcal{B}(I): I \in \mathcal{I}\}$ and the family is compatible, namely $E_{J} \uparrow \mathcal{B}(I)=E_{I}$ for $I, J \in \mathcal{I}$ with $I \subset J$.

4.1. Local hypergroup action. The construction in this section works for a general irreducible (finite depth or just extremal) finite index subfactor $A \subset B$ of type III, with the restriction that the canonical endomorphism $\gamma$ has no multiplicities.

We denote $\iota: A \rightarrow B$ the inclusion map, $\bar{\iota}: B \rightarrow A$ a conjugate and $v \in \operatorname{Hom}(\mathrm{id}, \iota \bar{\iota}) \subset B$ and $w \in \operatorname{Hom}(\mathrm{id}, \bar{\iota} \iota) \subset A$ isometries fulfilling the conjugate equations $\bar{\iota}\left(v^{*}\right) w=\iota\left(w^{*}\right) v=[B: A]^{-\frac{1}{2}} \cdot 1$.

Then $\Gamma=(\gamma=\iota \bar{\iota}, v, \iota(w))$ is a Q-system and the conditional expectation $E: B \rightarrow \iota(A) \subset B$ is given by the Q-system by $E(\cdot)=\iota(w)^{*} \gamma(\cdot) \iota(w)$ Lon94].

From now on we will assume that $\gamma$ has no multicities. By the following proposition, this is true if the dual canonical Q-system $\Theta=(\theta=\iota \bar{\iota}, w, x=\iota(v))$ is commutative.

Proposition 4.1. If $A \subset B$ is an irreducible finite index subfactor with $Q$-system $\Theta=(\theta, w, x)$ and the rigid $C^{*}$-tensor category $\langle\theta\rangle$ generated by $\theta$ has a braiding $\varepsilon(\cdot, \cdot)$, such that $\Theta$ is commutative, i.e. $\varepsilon(\theta, \theta) x=x$. Then $\operatorname{Hom}(\gamma, \gamma)$ is commutative, i.e. $\gamma$ has no multiplicities.

Proof. The algebra $Q=\operatorname{Hom}(\gamma, \gamma)=\gamma(A)^{\prime} \cap A$ is commutative if and only if $\gamma$ has no multiplicities. But we can use the commutativity of the Q-system $\Theta$ which implies that the convolution product on $\hat{Q}=\operatorname{Hom}(\theta, \theta)$ is commutative. Informally, using the Fourier transformation $\mathcal{F}: Q \rightarrow \hat{Q}$ we have 
$\mathcal{F}(a b)=\mathcal{F}(a) * \mathcal{F}(b)=\mathcal{F}(b) * \mathcal{F}(a)=\mathcal{F}(b a)$ for $a, b \in Q$. In the endomorphism notation using the Q-system property and the naturality of the braiding this looks like:

$$
\begin{aligned}
a b & =[M: N]^{3} \gamma\left(v^{*}\right) \iota\left(x^{*} w^{*}\right) \gamma(a \iota(w)) \gamma^{2}(b \iota(w)) \bar{\iota}(x) \\
& =[M: N]^{3} \gamma\left(v^{*}\right) \iota\left((\varepsilon(\theta, \theta) x)^{*} w^{*}\right) \gamma(a \iota(w)) \gamma^{2}(b \iota(w)) \bar{\iota}(\varepsilon(\theta, \theta) x) \\
& =[M: N]^{3} \gamma\left(v^{*}\right) \iota\left(x^{*} w^{*}\right) \gamma(b \iota(w)) \gamma^{2}(a \iota(w)) \bar{\iota}(x)=b a .
\end{aligned}
$$

We invite the reader to draw the diagram.

Corollary 4.2. Let $\mathcal{B}$ be a conformal net and $\mathcal{A}$ a finite index subnet, and consider the subfactor $A=\mathcal{A}(I) \subset B=\mathcal{B}(I)$. Then the canonical $\gamma \in \operatorname{End}(B)$ has no multiplicities.

Proof. The dual canonical Q-system $(\theta, w, x)$ is commutative by [LR95, 4.4. Corollary] and the statement follows from Proposition 4.1 .

Let us choose

$$
\gamma=\sum_{i=0}^{n} \operatorname{Ad}\left(v_{i}\right) \circ \beta_{i}
$$

a decomposition of $\gamma$ into irreducible sectors, where $v_{i} \in \operatorname{Hom}\left(\beta_{i}, \gamma\right)$ with $i=0, \ldots, n$ are representation the generators of the Cuntz algebra $\mathcal{O}_{n+1}$. We may and do choose $\beta_{0}=\mathrm{id}{ }_{B}$ and $v_{0}=v$. We remember that we assume that $\gamma$ has no multiplicities, thus $\left[\beta_{i}\right] \neq\left[\beta_{j}\right]$ if $i \neq j$ and $\operatorname{Hom}(\gamma, \gamma)=\gamma(B)^{\prime} \cap B$ is a commutative algebra.

Lemma 4.3. Let $(\gamma=\iota \bar{\iota}, v, \iota(w))$ be an irreducible Q-system. The maps

$$
\operatorname{Hom}\left(\beta_{i}, \gamma\right) \ni v_{i} \longmapsto \sqrt{\frac{d_{\gamma}}{d_{i}}} \begin{cases}v_{i}^{*} \iota(w) & \in \operatorname{Hom}\left(\iota, \beta_{i} \iota\right) \\ \bar{\iota}\left(v_{i}\right)^{*} w & \in \operatorname{Hom}\left(\bar{\iota}, \bar{\iota} \beta_{i}\right)\end{cases}
$$

are anti-isomorphism of the respective Hilbert spaces.

Proof. We have that $v_{i}^{*} \iota(w)=v_{i} \cdot\left(1_{\iota} \otimes w\right) \in \operatorname{Hom}\left(\iota, \beta_{i} \iota\right)$ is a multiple of an isometry. Then we get $\Phi_{\iota}\left(\iota(w) v_{i}^{*} v_{i} \iota(w)\right)=\Phi_{\gamma}\left(v_{i} v_{i}^{*}\right)=\frac{d_{i}}{d_{\gamma}} \Phi_{\beta_{i}}\left(v_{i}^{*} v_{i}\right)=\frac{d_{i}}{d_{\gamma}} \cdot 1$ using sphericality and the trace property.

Let us define normal completely positive maps $\phi_{i}$ by

$$
\frac{d_{i}}{d_{\gamma}} \phi_{i}(\cdot):=\iota\left(w^{*}\right) v_{i} \beta_{i}(\cdot) v_{i}^{*} \iota(w)=\iota\left(w^{*}\right) \gamma(\cdot) v_{i} v_{i}^{*} \iota(w)
$$

With this normalization, the maps $\phi_{i}: B \rightarrow B$ are also a unital maps. Namely, they are of the form $\phi_{i}=x_{i}^{*} \beta_{i}(\cdot) x_{i}$ with $x_{i}$ isometries by Lemma 4.3. It also follows directly that $\phi_{i} \circ \iota=\iota$. Namely,

$$
\frac{d_{i}}{d_{\gamma}} \phi_{i}(\iota(a))=\iota\left(w^{*}\right) v_{i} \beta_{i}(\iota(a)) v_{i}^{*} \iota(w)=\iota\left(w^{*}\right) \iota(\theta(a)) v_{i} v_{i}^{*} \iota(w)=\iota(a) \iota\left(w^{*}\right) v_{i} v_{i}^{*} \iota(w)=\frac{d_{i}}{d_{\gamma}} \iota(a)
$$


for all $a \in A$ using Lemma 4.3 . We have

$$
\begin{aligned}
\phi_{i} \circ \phi_{j}(\cdot) & =\frac{d_{\gamma}^{2}}{d_{i} d_{j}} \iota\left(w^{*}\right) v_{i} \beta_{i}\left(\iota\left(w^{*}\right) v_{j} \beta_{j}(\cdot) v_{i}^{*} \iota(w)\right) v_{i}^{*} \iota(w) \\
& =\frac{d_{\gamma}^{2}}{d_{i} d_{j}} \iota\left(w^{*}\right) \gamma(\cdot) \underbrace{\iota\left(w^{*}\right)\left(v_{i} \otimes v_{j}\right)\left(v_{i}^{*} \times v_{j}\right) \iota(w)}_{\in \operatorname{Hom}(\gamma, \gamma)} \iota(w) \\
& =\frac{d_{\gamma}}{d_{k}} \sum_{k} C_{i j}^{k} \cdot \iota\left(w^{*}\right) \gamma(\cdot) \underbrace{v_{k} v_{k}^{*}}_{\in \operatorname{Hom}(\gamma, \gamma)} \iota(w) \\
& =\sum_{k} C_{i j}^{k} \cdot \phi_{k}(\cdot)
\end{aligned}
$$

where in the last step we used that $\operatorname{Hom}(\gamma, \gamma) \cong \mathbb{C}^{\# K}$ with basis $v_{i} v_{i}^{*}$ and $\sum_{k} v_{k} v_{k}^{*}=1$. The coefficients $C_{i j}^{k}$ are given by:

$$
\begin{gathered}
C_{i j}^{k} v_{k} v_{k}^{*}=\frac{d_{\gamma} d_{k}}{d_{i} d_{j}} v_{k} v_{k}^{*}\left(\iota\left(w^{*}\right)\left(v_{i} \otimes v_{j}\right)\left(v_{i} \otimes v_{j}\right)^{*} \iota(w)\right) v_{k} v_{k}^{*} \\
C_{i j}^{k}=\frac{d_{\gamma} d_{k}}{d_{i} d_{j}}\left\|\left(v_{i}^{*} \otimes v_{j}^{*}\right) \cdot x \cdot v_{k}\right\|_{\operatorname{Hom}\left(\beta_{k}, \beta_{i} \beta_{j}\right)}^{2}=\frac{d_{\gamma} d_{k}}{d_{i} d_{j}}\left\|v_{i}^{*} \beta_{i}\left(v_{j}^{*}\right) x v_{k}\right\|_{\operatorname{Hom}\left(\beta_{k}, \beta_{i} \beta_{j}\right)}^{2},
\end{gathered}
$$

with the norm $\|a\|_{\operatorname{Hom}\left(\beta_{k}, \beta_{i} \beta_{j}\right)}^{2}=(a, a)_{\operatorname{Hom}\left(\beta_{k}, \beta_{i} \beta_{j}\right)}$.

Because $[\gamma]=[\bar{\gamma}]$ we can define the involution $i \mapsto \bar{i}$, such that $\left[\beta_{\bar{i}}\right]=\left[\bar{\beta}_{i}\right]$.

Proposition 4.4. Let $A \subset B$ be an irreducible finite index subfactor, such that the canonical endomorphism $\gamma$ has no multiplicities.

Then the coefficients $C_{i j}^{k}$ with $\phi_{i} \circ \phi_{j}=\sum_{k} C_{i j}^{k} \phi_{k}$ as above together with the involution $[i] \rightarrow[\bar{i}]$ (defined by $\left[\bar{\beta}_{i}\right]=\left[\beta_{\bar{i}}\right]$ ) defines a hypergroup $K=\left\{c_{0}, \ldots, c_{n}\right\}$.

There is a Haar element $e_{K} \in \mathbb{C} K$ defined by

$$
e_{K}=\frac{1}{d_{\gamma}} \sum_{i} w_{i} c_{i} \in \operatorname{Conv}(K)
$$

with $w_{i}=d_{i}$ the weight of $c_{i}$ and $D(K)=d_{\gamma} \equiv[B: A]$.

Proof. Since $\phi_{0}=\mathrm{id}_{B}$ and the composition is associative it follows that $\mathbb{C} K$ is a unital associative algebra. Property (1) is clear from the definition and (2) follows from the unital property by applying both sides to 1 . To see (3) we note that $i \neq \bar{j}$ we have that $\operatorname{Hom}\left(\beta_{0}, \beta_{i} \beta_{j}\right)=\{0\}$ and it follows from the standardness of $(v, w)$ that $C_{i \bar{i}}^{0}=1 / d_{i}$. Finally, we have to check the $*$-property. We may assume $\bar{\beta}_{i}=\beta_{\bar{i}}$. Using the rotation $v_{i} \mapsto v_{i}^{\bullet}$

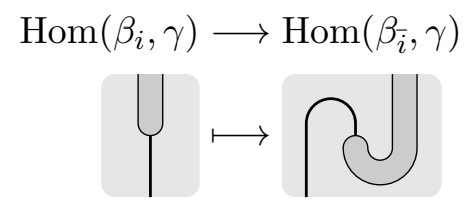

we get that $v_{i}$ coincides with $v_{i}^{\bullet}$ up to a phase. Then it is straight forward to show

$$
\phi_{\bar{j}} \phi_{\bar{i}}=\sum_{k} C_{i j}^{k} \phi_{\bar{k}}
$$

thus the desired property. 
The map $c_{i} \mapsto \phi_{i}$ defines (by linear extension) the map $\phi: \mathbb{C} K \rightarrow \operatorname{Maps}(B)$, which restricts to a map $\phi: \operatorname{Conv}(K) \rightarrow \operatorname{UCP}(B)$. Then the conditional expectation is by definition given by $E(\cdot)=\phi\left(e_{K}\right)(\cdot)$.

Let us assume we have a unit vector $\Omega$, which is cyclic and separating for $B$, such that $(\Omega, E(b) \Omega)=$ $(\Omega, b \Omega)$, i.e. the conditional expectation $E$ preserves the vector state $\varphi=(\Omega, \Omega)$.

Lemma 4.5. Let $x_{i}=\sqrt{d_{\gamma} / d_{i}} v_{i}^{*} \iota(w)$. Then $\phi_{i}=x_{i}^{*} \beta_{i}(\cdot) x_{i}$ is a minimal Stinespring representation, i.e. $\overline{\beta_{i}(B) x_{i} \mathcal{H}}=\mathcal{H}$

Proof. We have to show that the set $\mathcal{V}=\overline{\beta_{i}(B) x_{i} \mathcal{H}_{B}}$ equals $\mathcal{H}_{B}$. But the space $\mathcal{V}$ is invariant under $\beta_{i}(B)$ and $B^{\prime}$ (even $\left\langle\beta(B), x_{i}\right\rangle$ ). Thus the projection onto $\mathcal{V}$ is in $\beta_{i}(B)^{\prime} \cap B=\mathbb{C}$ which proves the statement.

We remember that a normal unital complete positive map $\phi$ with $\varphi \circ \phi=\varphi$ is called a $\Omega$ preserving stochastic map and $\Omega$-adjoint is $\Omega$-preserving stochastic map $\phi^{\sharp}$ fulfilling $\varphi\left(\phi^{\sharp}(a) b\right)=$ $\varphi(a \phi(b))$ for all $a, b \in B$.

It turns out that already the representation of the conditional expectation using the Q-system is a minimal Stinespring representation.

Lemma 4.6. $E(\cdot)=\iota(w)^{*} \gamma(\cdot) \iota(w): B \rightarrow A \subset B$ is the minimal Stinespring representation.

Proof. We have $\gamma\left(v^{*}\right) \iota(w)=\iota\left(\bar{\iota}\left(v^{*}\right) w\right)=d_{\gamma}^{-\frac{1}{2}} \cdot 1$ because $(v, w)$ is a standard solution of the conjugate equation for $\gamma$. But then $\gamma(B) \iota(w) \mathcal{H} \supset \gamma\left(v^{*}\right) y \mathcal{H}=\mathcal{H}$, thus the representation is minimal.

Lemma 4.7. We have $E\left(a \phi_{k}(b)\right)=E\left(\phi_{\bar{k}}(a) b\right)$ and $E \circ \phi_{k}=E$. In particular, $\phi_{k} \in \mathrm{Stoch}_{\Omega}(B)$ and $\phi_{k}^{\sharp}=\phi_{\bar{k}}$.

Proof. Using the trace property and rotation as in 22) we have:

$$
\begin{aligned}
E\left(a \phi_{k}(b)\right) & =E\left(a x_{k}^{*} \beta_{k}(b) x_{k}\right) \\
& =E\left(R_{k}^{*} \beta_{\bar{k}}\left(x_{k}^{*} a x_{k} \beta_{k}(b)\right) R_{k}\right) \\
& =E\left(R_{k}^{*} \beta_{\bar{k}}\left(x_{k}^{*} a x_{k}\right) R_{k} b\right) \\
& =E\left(x_{\bar{k}}^{*} \beta_{\bar{k}}(a) x_{\bar{k}} b\right) \\
& =E\left(\phi_{\bar{k}}(a) b\right),
\end{aligned}
$$

where $d_{k}^{-\frac{1}{2}} R_{k}$ is the up to phase unique isometry in Hom(id, $\left.\beta_{\bar{k}} \beta_{k}\right)$. Using Lemma 4.3 we get

$$
E \circ \phi_{k}(\cdot)=\frac{d_{\gamma}}{d_{k}} \iota(w)^{*} \gamma\left(\iota(w)^{*} \gamma(\cdot) v_{k} v_{k}^{*} \iota(w)\right) \iota(w)=\frac{d_{\gamma}}{d_{k}} \iota(w)^{*} \gamma(\cdot) \underbrace{\iota\left(w^{*} \bar{\iota}\left(v_{k} v_{k}^{*}\right) w\right)}_{=\frac{d_{k}}{d_{\gamma}}} \iota(w)=E(\cdot) \text {. }
$$

Lemma 4.8. The stochastic maps $\left\{\phi_{k}\right\}_{k=0}^{n}$ are affine independent, i.e. $\left\{\phi_{k}-\phi_{0}\right\}$ are linearly independent.

Proof. Define $b_{k}=\phi_{\bar{k}}\left(v v^{*}\right) \in B$ for $1 \leq k \leq n$. We have that $\phi_{0}\left(b_{k}\right) v \equiv m_{k} v=0$. Then $\phi_{\ell}\left(b_{k}\right) v=\delta_{k, \ell} c_{k} v$ for some positive numbers $c_{k}$. This shows that $\left\{\phi_{k}-\phi_{0}\right\}_{1 \leq k \leq n}$ are linearly independent.

Remark 4.9. Since $E\left(v v^{*}\right)=[B: A]^{-1} \cdot 1$ it follows that with $\xi=[B: A]^{\frac{1}{2}} \cdot v v^{*} \Omega$ the map $\mathbb{C} K \ni x \mapsto$ $(\xi, V(x) \xi)$ gives the normalized trace on $\mathbb{C} K$. Namely, $\left(\xi, V_{k} \xi\right)=[B: A] \cdot\left(\Omega, v v^{*} \phi_{k}\left(v v^{*}\right) \Omega\right)=\delta_{k, 0}$.

Proposition 4.10. The convex space $\operatorname{Conv}\left(\left\{\phi_{0}, \ldots, \phi_{n}\right\}\right)$ is an $n$-simplex in $\operatorname{Stoch}_{\Omega}(B)$. It coincides with the space of all $A-A$ bimodular maps in $\operatorname{Stoch}_{\Omega}(B)$. 
Proof. Using Lemma 4.5 and Proposition A.5 it follows that $\phi_{k}$ are extreme points in $\operatorname{Stoch}_{\Omega}(B)$. By Lemma 4.8 they are affine independent.

For the second statement it is enough to show that the space of $A-A$ bimodular maps has dimension $n+1$. We have the unique Fourier decomposition $b=\sum \iota\left(b_{\rho, i}\right) \psi_{\rho, i}$ as before. Let $\phi: B \rightarrow B$ be a $A-A$ bimodular map. From $\phi\left(\psi_{\rho, i}\right) \iota(a)=\phi\left(\psi_{\rho, i} \iota(a)\right)=\phi\left(\iota \rho(a) \psi_{\rho, i}\right)=\iota \rho(a) \phi\left(\psi_{\rho, i}\right)$ for all $a \in A$ follows $\phi\left(\psi_{\rho, i}\right) \in \operatorname{Hom}(\iota, \iota \rho)$ and $\phi$ is determined by $\operatorname{dim} \operatorname{Hom}(\theta, \theta)=\operatorname{dim} \operatorname{Hom}(\gamma, \gamma)=$ $n+1$ coefficients.

Theorem 4.11. Let $(A \subset B, \Omega)$ be an irreducible finite index type III subfactor $A \subset B$, such that the dual canonical endomorphism has no multiplicities. Let $\Omega$ be cyclic and separating for $B$, such that $(\Omega, E(\cdot) \Omega)=(\Omega, \cdot \Omega)$ for the unique conditional exptectation $E: B \rightarrow A \subset B$. Then there is a canonical hypergroup $K$ and a $\Omega$-preserving normal faithful action on $B$, such that $A=B^{K}$.

Let $\tilde{K}$ be another $\Omega$-preserving faithful normal action, such that $B^{\tilde{K}}=A$, then there is an isomorphism $\tau: K \rightarrow \tilde{K}$, such that $\tilde{\phi}_{\tau(k)}=\phi_{k}$.

Proof. The unique conditional expectation $E: B \rightarrow \iota(A) \subset B$ can be written as $E=\operatorname{Ad} \iota(w)^{*} \gamma$ and every pure UCP map corresponds to a minimal projection $p \in \operatorname{Hom}(\gamma, \gamma) \cong \mathbb{C}^{k}$ and is independent of the choice of decomposition of $\gamma=\sum \operatorname{Ad} v_{k} \circ \beta_{k}$. If we choose a different Q-system $(\tilde{\gamma}, \tilde{v}, \tilde{y})$ associated with $(A \subset B, \Omega)$, then there is [Lon94] a unitary $u \in \operatorname{Hom}(\gamma, \tilde{\gamma})$, such that $u \tilde{v}=v$ and $\tilde{y} u=(u \otimes u) y$. Finally, (1) does not depend on the choice of the Q-system, since Ad $u$ maps minimal projection to minimal projections.

The conditional expectation is unique which implies $D_{K}^{-1} \sum_{k} d_{k} \phi_{k}=D_{\tilde{K}}^{-1} \sum_{\tilde{k}} d_{\tilde{k}} \tilde{\phi}_{\tilde{k}}$. By Proposition $3.4 \tilde{\phi}_{\tilde{k}}$ are $A-A$ bimodular and by assumption they are extremal and affine independent. Therefore, by Proposition 4.10 there is a $\tau: K \rightarrow \tilde{K}$, such that $\tilde{\phi}_{\tau(k)}=\phi_{k}$.

Example 4.12. Let $\mathcal{F} \equiv{ }_{N} \mathcal{F}_{N} \subset \operatorname{End}(N)$ with $N$ a type III factor (e.g. the hyperfinite type $\mathrm{III}_{1}$ factor) and $\operatorname{Irr}(\mathcal{F})=\left\{\left[\rho_{0}\right], \ldots,\left[\rho_{n}\right]\right\}$ be a UFC, and $B:=N \otimes N^{\mathrm{op}}$. Let $\gamma \in \operatorname{End}(B)$ be the canonical endomorphism associated with the Longo-Rehren Q-system $(\gamma, v, z)$, see Section 2.4. Let $A=E(B) \subset B$ be the Longo-Rehren inclusion with conditional expextation $E(\cdot)=z^{*} \gamma(\cdot) z$. Then $\beta_{i}=\rho_{i} \otimes \rho_{i}^{\mathrm{op}}, w_{i}=\left(d \rho_{i}\right)^{2}$ and direct calculation shows:

$$
C_{i j}^{k}=\sqrt{\frac{w_{k}}{w_{i} w_{j}}} N_{i j}^{k}=\frac{d \rho_{k}}{d \rho_{i} d \rho_{j}} \operatorname{dim} \operatorname{Hom}\left(\rho_{k}, \rho_{i} \rho_{j}\right) .
$$

In particular, we have an action of $K_{\mathcal{F}}$ on $B$, such that $A=B^{K_{\mathcal{F}}}$. From a cyclic and separating vector $\xi$ for $B$, we get a vector $\Omega$, such that $(\Omega, b \Omega)=(\xi, E(b) \xi)$ for all $b \in B$ and we get therefore a $\Omega$-preserving faithful action of $K_{\mathcal{F}}$ on $B$.

4.2. Graphical Representation of Stochastic Maps. Using the graphical calculus as in BKLR16 we draw the conditional expectation as:

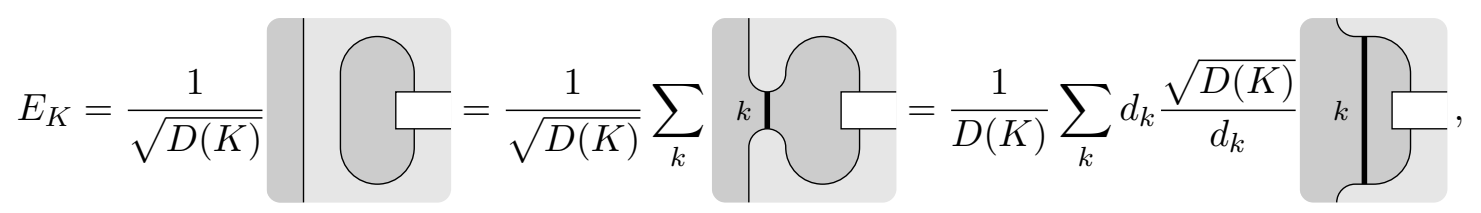

where we use the convention that the vertices are normalized to be isometries in $\operatorname{Hom}\left(\beta_{k}, \iota \bar{\iota}\right)$. The stochastic maps $\phi_{k}$ and $\phi_{i} \circ \phi_{j}$ are therefore represented as:

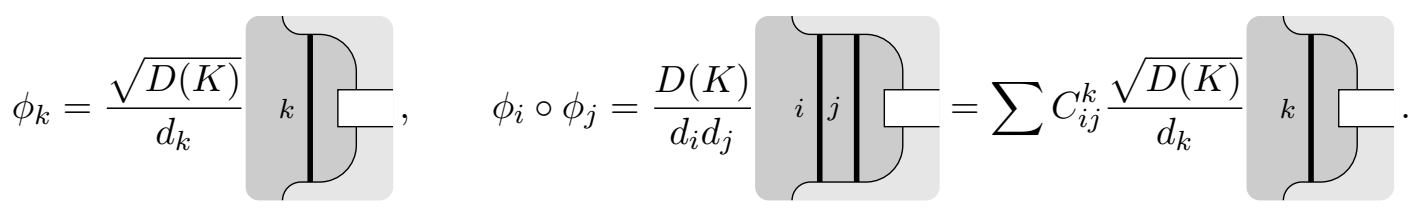


Here we used that the coefficients (1) are given by:

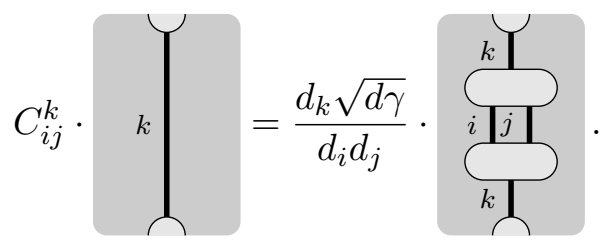

4.3. Galois Theory. Let $A \subset B$ be an irreducible finite index type III subfactor with canonical endomorphism $\gamma=\iota \bar{\iota} \in \operatorname{End}(B)$ and Q-system $(\gamma, v, \iota(w))$. There is a one-to-one correspondence between projections $P \in \operatorname{Hom}(\gamma, \gamma)$ with

$$
P v=v, \quad \iota(w)^{*}(P \otimes P) \iota(w)=\lambda \cdot P
$$

for some $\lambda>0$ [ILP98, BKLR15] and intermediate factors $M$ with $A \subset M \subset B$. In this case $\lambda=[M: A]^{-1}$.

Let us assume that $\iota(A) \subset B$ has an intermediate factor $M$, i.e. $\iota(A) \subset \iota_{M}(M) \subset B$. Then we have the subfactors $\iota_{M}(M) \subset B$ and $\iota_{A}(A) \subset M$ with $\iota_{A}=\iota_{M}^{-1} \circ \iota$, thus $\iota=\iota_{M} \circ \iota_{A}$.

Proposition 4.13. Let $K$ be the canonical hypergroup associated with $(A \subset B, \Omega)$. (Then $A=B^{K}$.) There is a one-to-one correspondence between

- subhypergroups $L \subset K$ and

- intermediate subfactors $A \subset M \subset B$

given by $M=B^{L}$ and $L=\left\{k \in K: \phi_{k}(m)=m\right.$ for all $\left.m \in M\right\}$.

The conditional expectation is given by $E_{L}=\phi\left(e_{L}\right)$, where $e_{L}=\frac{1}{D(L)} \sum_{k \in L} w_{k} c_{k}$ is the Haar element of $L$.

Proof. Let $L \leq K$, then $L$ corresponds to a unique projection $P \in \operatorname{Hom}(\gamma, \gamma) \cong \mathbb{C}^{|K|}$ and since $L$ is a subhypergroup, i.e. $c_{0} \in L$ and $L L \subset \mathbb{C} L$, we have 4.3). Conversely, given an intermediate subfactor $M$, it corresponds to a projection $P \in \operatorname{Hom}(\gamma, \gamma)$ corresponding to a subsector $\gamma_{P} \prec \gamma$ and therefore to a subset $L \subset K$. We have $L^{*}=L$ since $\gamma_{P}$ has the structure of a Q-system and is therefore self-dual. Finally, 4.3 gives $L L \subset \mathbb{C} L$.

Let $e_{L}=\frac{1}{D(L)} \sum_{c_{l} \in L} w_{l} c_{l}$ and $E_{L}=\phi\left(e_{L}\right)$ then $\phi_{l} \circ E_{L}=E_{L}$ for $c_{l} \in L$ and therefore $L \subset\{k \in$ $K: \phi_{k}(m)=m$ for all $\left.m \in M\right\}$. Conversely, to see $L \supset\left\{k \in K: \phi_{k}(m)=m\right.$ for all $\left.m \in M\right\}$ we note that for $k \in K \backslash L$, we have $E_{L} \circ \phi_{k} \circ E_{L} \neq E_{L}$. Namely, since the representation of $\mathbb{C} K$ is faithful it is enough to show that $e_{L} c_{k} e_{L} \neq e_{L}$, which follows from Proposition 2.16. It independently follows from the proof of the following Proposition 4.16 .

By construction the conditional expectation $E_{L} \equiv \phi\left(e_{L}\right)$ coincides with the conditional expectation $E_{M}$ onto $M$.

Let $K$ be a hypergroup and $F \subset K$ a subset, we denote by $\langle F\rangle$ the subhypergroup generated by $F$, i.e. the smallest subhypergroup containing $F$. It follows easily that $\langle F\rangle=\left\{c_{k} \in K: c_{k} \prec\right.$ finite word in $F \cup F^{*}$ \}

Corollary 4.14. If $K=\left\langle c_{k}\right\rangle$, then $M^{K}$ equals $M^{\phi_{k}}=\left\{m \in M: \phi_{k}(m)=m\right\}$.

Proof. We have that $M^{K} \subset M^{\phi_{k}} \subset M$, but by Proposition 4.13 there is a subhypergroup $L \subset K$, such that $M^{\phi_{k}}=M^{L}$ and since $c_{k}$ generates $K$ it follows from the proof that $c_{k} \in L$ and therefore $K=L$.

Lemma 4.15. Let $\iota(A) \subset B$, such that $\gamma=\iota \bar{\iota}$ has no multiplicities, and $\iota_{M} \iota_{A}(A) \subset \iota_{M}(M) \subset B$ an intermediate subfactor, i.e. $\iota_{M} \iota_{A}=\iota$. Then $\gamma_{A}=\iota_{A} \bar{\iota}_{A}$ associated with $\iota_{A}(A) \subset M$ has no multiplicities. 
Proof. Suppose $\gamma_{A}$ multiplicities, i.e. there is an irreducible $\beta$ with $\left\langle\beta, \gamma_{A}\right\rangle>1$. There is always an irreducible $\beta_{B}$ and a non-trivial $t \in \operatorname{Hom}\left(\beta_{B}, \iota_{M} \beta \iota_{M}\right)$. But then with the embedding $\operatorname{Hom}\left(\beta, \gamma_{A}\right) \ni$ $w \mapsto\left(\iota_{M} \otimes w \otimes \bar{\iota}_{M}\right) \cdot t \in \operatorname{Hom}\left(\beta_{B}, \gamma\right)$, we get $\left\langle\beta_{B}, \gamma\right\rangle \geq\left\langle\beta, \gamma_{A}\right)>1$ which is a contradiction.

Proposition 4.16. Let $K$ be the canonical hypergroup associated with $(A \subset B, \Omega)$ and therefore $A \equiv B^{K}$ and $L \leq K$ a subhypergroup.

Then there is a natural action of $K / / L$ on $M \equiv B^{L}$, which coincides with canonical action of the hypergroup aossciated with $\left(A \subset M \equiv B^{L}, \Omega\right)$. In particular, $A=M^{K / / L}=\left(B^{L}\right)^{K / / L}$ and the weights fulfill $D(K)=D(L) \cdot D(K / / L)$.

Proof. Let $L \leq K$ be subhypergroup. Let us write $[k]=e_{L} c_{k} e_{L} \in K / / L$. Since $\phi([k])=E_{L} \circ \phi_{k} \circ E_{L}$ the map $\tilde{\phi}_{[k]}=\iota_{L}^{-1} \circ E_{L} \circ \phi_{k} \circ \iota_{L}$ is well-defined, where $\iota_{L}$ is the canonical inclusion of $B^{L}$ into $B$. It follows directly that this fulfills properties of an action of $K / / L$ on $M$.

By Lemma $4.15 \tilde{\gamma}=\iota_{A} \bar{\iota}_{A}$ has no multiplicities and we get a hypergroup $\tilde{L}$ with an action $\psi: \tilde{L} \rightarrow \operatorname{Stoch}_{\Omega}(M)$, such that $\iota_{A}(A) \equiv M^{\tilde{L}} \subset M$.

Let $[\tilde{\gamma}]=\bigoplus_{l \in \tilde{L}}\left[\tilde{\beta}_{l}\right]$, then $[\gamma]=\bigoplus_{k \in K}\left[\beta_{k}\right]=\bigoplus_{l \in \tilde{L}}\left[\iota_{M} \tilde{\beta}_{l} \bar{\iota}_{M}\right]$. Since $\gamma$ and $\tilde{\gamma}$ have no multiplicities, we get a surjective map $\ell: K \rightarrow \tilde{L}$ fixed by the requirement that $\beta_{k} \prec \iota_{M} \tilde{\beta}_{\ell(k)} \bar{\iota}_{M}$ for all $k \in K$. The conditional expectation onto $A$ factorizes as follows:

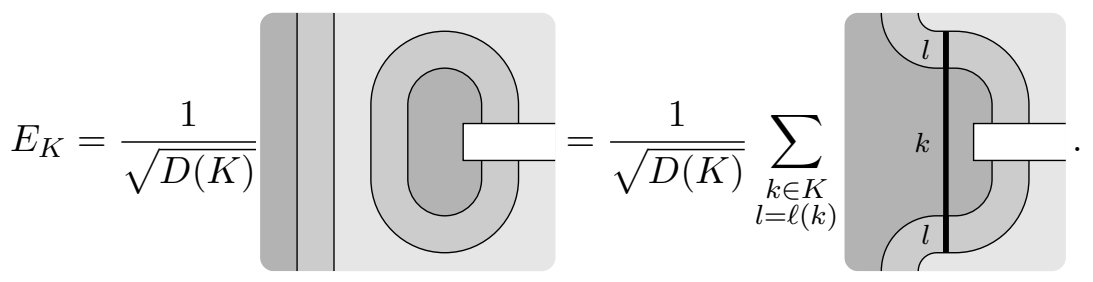

This gives

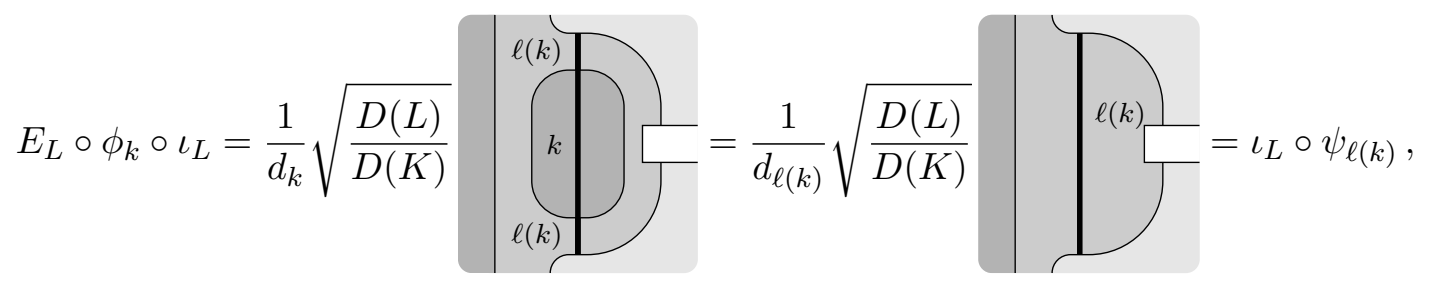

which shows that $\ell$ factors through to be a bijection $\ell: K / / L \rightarrow \tilde{L}$ and that $\tilde{\phi}_{[k]}=\psi_{\ell(k)}$.

Remark 4.17. The proof implies that $K / / L$ is a hypergroup without using Proposition 2.16. The proof also shows that we have an exact sequence:

$$
\left\{c_{0}\right\} \longrightarrow L \longrightarrow K \longrightarrow K / / L \longrightarrow\left\{\tilde{c}_{0} \equiv e_{L}\right\} .
$$

4.4. Nilpotent Hypergroups and Intermediate Groups. We can ask if the intermediate inclusions are coming from group fixed points. Let $K$ be a hypergroup. We remember that a $K$ is graded by $G_{K}=K / / K_{\text {ad }}$, where $K_{\text {ad }}=\left\{c_{l} \prec c_{k} c_{\bar{k}}\right.$ for $\left.c_{k} \in K\right\}$ is the adjoint hypergroup.

By iteratively taking the adjoint hypergroup, we get the following finite sequence of proper subhypergroups:

$$
K_{0}=K \supsetneq K_{1}=K_{\text {ad }} \supsetneq K_{2}=\left(K_{1}\right)_{\text {ad }} \supsetneq \cdots \supsetneq K_{N}=\left(K_{N-1}\right)_{\text {ad }}, \quad K_{i+1}=\left(K_{i}\right)_{\text {ad }},
$$

with $\left(K_{N}\right)_{\text {ad }}=K_{N}$. We get a sequence $\left(G_{i}\right)_{1 \leq i \leq N}$ of finite groups given by

$$
G_{i}=K_{i-1} / / K_{i}, \quad 1 \leq i \leq N .
$$

Then the weight of $K$ is given by $D(K)=\left|G_{1}\right|\left|G_{2}\right| \cdots\left|G_{N}\right| \cdot D\left(K_{N}\right)$. The hypergroup $K$ is called nilpotent (cf. [EGNO15, Definition 3.6.7]) if $K_{N}=\left\{c_{0}\right\}$ for some $N \geq 0$. 
Corollary 4.18. If $K$ is the canonical hypergroup associated with $(A \subset B, \Omega)$, i.e. $A=B^{K}$, then by applying Proposition 4.16 recursively, we get

$$
B^{K}=\left(\cdots\left(\left(B^{K_{N}}\right)^{G_{N}}\right)^{G_{N-1}} \cdots\right)^{G_{1}} .
$$

In particular, if $K$ is nilpotent, then $D(K)=\left|G_{1}\right|\left|G_{2}\right| \cdots\left|G_{N}\right|$ and $M_{K}$ is an iterated group fixed point algebra

$$
M^{K}=\left(\cdots\left(M^{G_{N}}\right)^{G_{N-1}} \cdots\right)^{G_{1}} .
$$

Example 4.19. Let $G$ be an abelian group and $\mathcal{F}$ be a unitary fusion category of TambaraYamagami type (see TY98, Izu01]), i.e. $\operatorname{Irr}(\mathcal{F})=G \cup\{\rho\}$ with fusion rules:

$$
[\rho]^{2}=\bigoplus_{g \in G}[g], \quad[g][\rho]=[\rho][g]=\rho, \quad[g][h]=[g h], \quad \text { for all } g, h \in G .
$$

Then the universal grading group of $K_{\mathcal{F}}$ is $G_{1}=G_{K_{\mathcal{F}}} \cong \mathbb{Z}_{2}$ and $G_{2} \cong A$. Let $N \subset M$ be the Longo-Rehren inclusion associated with $\mathcal{F}$. Then $N=\left(M^{G}\right)^{\mathbb{Z}_{2}}$, this means $N \subset M$ is a BischHaagerup subfactor (see $\mid \mathrm{BH} 96) P^{\mathbb{Z}_{2}} \subset P \rtimes \hat{G}$. Here $P=M^{A}$ and $\hat{G}$ is the Pontryagin dual of $G$.

Let $K$ be a hypergroup and let $K^{\times}=\left\{c_{k}: w_{k}=1\right\} \subset K$ be the maximal subgroup or unit ring of $K$, i.e. the maximal subhypergroup which is a group.

Corollary 4.20. If $K$ is the canonical hypergroup associated with $(A \subset B, \Omega)$, i.e. $A=B^{K}$, such that $G:=K^{\times}$is non-trivial. Then

$$
A=\left(B^{G}\right)^{K / / G} .
$$

4.5. A Finite Index Subnet Gives a Proper Hypergroup Action. As before, let $\mathcal{A} \subset \mathcal{B}$ be a finite index inclusion of conformal nets. In this subsection we want to show, that this gives rise to a proper action of a hypergroup. We consider $A=\mathcal{A}(I) \subset \mathcal{B}(I)=B$ and by Corollary 4.2 the canonical endomorphism $\gamma=\iota \bar{\iota}$ has no multiplicities. From $(A \subset B, \Omega)$, we get canonically a hypergroup $K$ and stochastic maps: $\phi_{k}^{I} \equiv \phi_{k}: B \rightarrow B$.

Indeed we get a compatible family indexed by intervals $I_{1} \in \mathcal{I}$ of such actions and hence giving a converse of Theorem 3.8 , see Theorem 4.22 .

Proposition 4.21. $\phi_{k}$ extends to a compatible and vacuum preserving family $\left\{\phi_{k}^{I_{1}}: \mathcal{B}\left(I_{1}\right) \rightarrow \mathcal{B}\left(I_{1}\right)\right.$ : $\left.I_{1} \in \mathcal{I}\right\}$.

Proof. We remember that every element in $b \in B$ can be written as

$$
b=\iota(a) v=\sum_{\rho, e} \iota\left(a_{\rho, e}\right) \psi_{\rho, e}
$$

with $\left\{\psi_{\rho, e}\right\}_{e=1}^{\langle\iota, \iota \rho\rangle}$ an orthonormal basis of $\operatorname{Hom}(\iota, \iota \rho)$. Then $\phi_{k}\left(\psi_{\rho, e}\right) \in \operatorname{Hom}(\iota, \iota \rho)$. Let us now take a $I_{1} \subset I$. For $\rho \in \operatorname{Rep}^{I}(\mathcal{A})$ take a sinitary $u \in \operatorname{Hom}\left(\rho, \rho^{I_{1}}\right)$ with $\rho^{I_{1}}$ localized in $I_{1}$. Then $\iota(u) \psi_{\rho, e} \in \mathcal{B}\left(I_{1}\right)$ (cf. [LR95, below 4.6 Corollary]).

But then also $\phi_{k}\left(\iota(u) \psi_{\rho, e}\right)=\iota(u) \phi_{k}\left(\psi_{\rho, e}\right) \in \mathcal{B}\left(I_{1}\right)$, thus by linearity and $A$-bimodularity, we conclude that $\phi_{k}^{I_{1}}:=\phi_{k}\left\lceil\mathcal{B}\left(I_{1}\right): \mathcal{B}\left(I_{1}\right) \rightarrow \mathcal{B}\left(I_{1}\right)\right.$ is well-defined.

To show that we can extend a $\phi_{k}^{I_{1}}: \mathcal{B}\left(I_{1}\right) \rightarrow \mathcal{B}\left(I_{1}\right)$ to $\phi_{k}^{I_{2}}: \mathcal{B}\left(I_{2}\right) \rightarrow \mathcal{B}\left(I_{2}\right)$ for all $I_{1} \subset I_{2}$ with $I_{i} \in \mathcal{I}$ in a compatible way, we assume wlog $I_{1}=I$. By [LR95, 3. Thereom] $\gamma \in \operatorname{End}(B)$ can be extended to $\gamma^{I_{2}} \in \operatorname{End}\left(\mathcal{B}\left(I_{2}\right)\right)$, such that $\gamma^{I_{2}} \uparrow B^{\prime} \cap \mathcal{A}\left(\overline{\left.I_{2}\right)}=\mathrm{id}\right.$ and $\gamma^{I_{2}} \uparrow B=\gamma$. But then 
we can define with $\iota_{I_{2}}: \mathcal{A}\left(I_{2}\right) \rightarrow \mathcal{B}\left(I_{2}\right)=\iota_{I_{2}}\left(\mathcal{A}\left(I_{2}\right)\right) v$ the canonical inclusion with $\iota_{I_{2}} \uparrow A=\iota$ the compatible extension:

$$
\begin{aligned}
\phi_{i}^{I_{2}}(\cdot) & =\frac{d_{\gamma}}{d_{i}} \iota_{I_{2}}\left(w^{*}\right) \gamma^{I_{2}}(\cdot) v_{i} v_{i}^{*} \iota_{I_{2}}(w), \\
\phi_{i}^{I_{2}} \uparrow B & =\frac{d_{\gamma}}{d_{i}} \iota\left(w^{*}\right) \gamma(\cdot) v_{i} v_{i}^{*} \iota(w) \\
& =\frac{d_{\gamma}}{d_{i}} \iota\left(w^{*}\right) v_{i} \beta_{i}(\cdot) v_{i}^{*} \iota(w) \\
& =\phi_{i}(\cdot), \\
\phi^{I_{2}} \circ \iota_{2}(\cdot) & =\frac{d_{\gamma}}{d_{i}} \iota_{I_{2}}\left(w^{*}\right) \gamma^{I_{2}} \circ \iota_{I_{2}}(\cdot) v_{i} v_{i}^{*} \iota_{I_{2}}(w) \\
& =\frac{d_{\gamma}}{d_{i}} \iota_{2}(\cdot) \iota_{I_{2}}\left(w^{*}\right) v_{i} v_{i}^{*} \iota_{I_{2}}(w) \\
& =\frac{d_{\gamma}}{d_{i}} \iota_{2}(\cdot) \iota\left(w^{*}\right) v_{i} v_{i}^{*} \iota(w) \\
& =\iota_{2}(\cdot),
\end{aligned}
$$

where in last step we have used [LR95, 3.6 Lemma].

It follows directly that $K$ and the representation $V: \mathbb{C} K \rightarrow \mathrm{B}(\mathcal{H})$ do not depend on the choice of the interval.

Theorem 4.22. Let $\mathcal{B}$ be a conformal net on $\mathcal{H}$ and $\mathcal{A} \subset \mathcal{B}$ a be finite index subnet.

Then associated with $\mathcal{A}$ there is canonical hypergroup $K$ acting properly on $\mathcal{B}$, such that $\mathcal{A}=\mathcal{B}^{K}$. Further, there is $*$-representation of $\mathbb{C} K$ on $\mathcal{H}$, such that $\mathcal{A}(I)=\mathcal{B}(I) \cap V(\mathbb{C} K)^{\prime}$ and $\mathcal{H}$ decomposes as $\mathbb{C} K$ module as

$$
\mathcal{H}=\bigoplus_{\pi} \mathcal{H}_{\pi}, \quad \mathcal{H}_{\pi} \cong \mathcal{K}_{\pi} \otimes \mathcal{M}
$$

with $\mathcal{H}_{\text {trivial }}=\overline{\mathcal{A}(I) \Omega}$, where the sum is index by finite dimensional irreducible $*$-representations $\pi$ of $\mathbb{C} K$ on $\mathcal{K}_{\pi}$.

Proof. By Theorem 4.11, we get an action on $B=\mathcal{B}(I)$, which extends to the net by Proposition 4.21 .

We have that every element in $B$ can be uniquely written as $b=\sum_{\rho \prec \theta} \sum_{i=1}^{\langle\iota, \iota \rho\rangle} \iota\left(a_{\rho, i}\right) \psi_{\rho, i}$, where $\left\{\psi_{\rho, i}: i=1, \ldots,\langle\iota, \iota \rho\rangle\right\}$ is a orthonormal basis of the finite dimensional Hilbert space $\operatorname{Hom}(\iota, \iota \rho)$. Then there is a $*$-representation $\pi_{\rho}$ of $\mathbb{C} K$ on the finite dimensional Hilbert space $\operatorname{Hom}(\iota, \iota \rho)$ given by bilinearly extending $\pi_{\rho}(k) \psi_{\rho, j}=\phi_{k}\left(\psi_{\rho, j}\right)$. Then $\mathcal{H}_{\pi_{\rho}}=\bigoplus_{i=1}^{\langle\iota, \iota \rho} \overline{\iota(A) \psi_{\rho, i} \Omega}$ and $\mathcal{H}=\bigoplus_{\rho \prec \theta} \mathcal{H}_{\pi_{\rho}}$ gives the decomposition. On the dense domain $B \Omega$ we have

$$
\left.V(k) b \Omega \equiv \sum_{\rho \prec \theta} \phi_{k}\left(\iota\left(a_{\rho, i}\right) \psi_{\rho, i}\right) \Omega=\sum_{\rho \prec \theta} \iota\left(a_{\rho, i}\right)\right) \psi_{\rho, i} \Omega, \quad b=\sum_{\rho \prec \theta} \sum_{i=1}^{\langle\iota, \iota \rho\rangle} \iota\left(a_{\rho, i}\right) \psi_{\rho, i} .
$$

Remark 4.23. We remark that $\mathbb{C} K$ is isomorphic to the algebra $\operatorname{Hom}(\theta, \theta)=\theta(A)^{\prime} \cap A$ and $\pi_{\rho}$ is the representation on the Hilbert space $\operatorname{Hom}(\rho, \theta)$. Using the Fourier transformation, we get $\mathbb{C} K \cong \operatorname{Hom}(\gamma, \gamma)=\gamma(B)^{\prime} \cap B$ as vector spaces (where the multiplication is a convolution product). In the special case, that $\theta$ has no multiplicities we get a hypergroup $\hat{K}$ for the inclusion $\gamma(B) \subset \iota(A)$, such that $\mathbb{C} \hat{K} \cong \operatorname{Hom}(\gamma, \gamma)$. If $\hat{K}$ is a (necessarily abelian finite) group, then $\mathcal{H}$ is graded by $\hat{K}$ and 
we get the usual Fourier transformation (Pontryagain dualiy) for a fixed point $A=B^{G} \subset B$ of an action of a finite abelian group $G=K$.

4.6. Examples. The easiest non-trivial hypergroup $K=\left\{c_{0}=1, c_{1}=c\right\}$ has two elements and is generated by $c_{1}$. Since it is generated by $c_{1}$ multiplication with $c_{1}$ defines a Markov chain on $K$ see Figure 1. It is given by:

$$
c_{1} c_{1}=\frac{1}{d} c_{0}+\frac{d-1}{d} c_{1} .
$$

It arises from an inclusion of a subfactor $(A \subset B, \Omega)$ (or a finite index inclusion of conformal nets choosing $A=\mathcal{A}(I) \subset B:=\mathcal{B}(I))$ with $d=[B: A]-1$, if and only if the canonical endomorphism $\gamma$ is given by $[\gamma]=\left[\beta_{0}=\operatorname{id}_{B}\right] \oplus\left[\beta_{1}\right]$ with $\beta:=\beta_{1}$ irreducible and $d \beta=d$. In other words, we assume that $\langle\gamma, \gamma\rangle=\langle\theta, \theta\rangle=2$. This means the subfactor $A \subset B$ is $n$-supertransitive (as defined in [JMS14]) for some $n \geq 2$. In the case of an action of $K=\left\{c_{0}=1, c_{1}=c\right\}$ on a completely rational

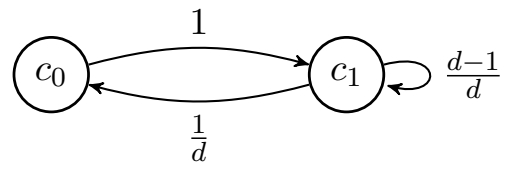

FIGURE 1. Markov chain of hypergroup $K=\left\{c_{0}, c_{1}\right\}$ obtained by multiplying with $c_{1}$

net $\mathcal{B}$, we get by Corollary 4.14 that

$$
\mathcal{A}(I)^{K}=\mathcal{A}(I)^{\phi^{I}}
$$

where $\phi^{I}=\phi_{1}^{I}$ and $\phi_{0}^{I}=\operatorname{id}_{\mathcal{B}(I)}$ are the stochastic maps. In Table 1 we list some known examples. Many of the examples come from intermediate inclusion as in Corollary 4.20 .

\begin{tabular}{lll}
$d$ & $\mathcal{B}^{K} \subset \mathcal{B}$ & $K$ \\
\hline$(3+\sqrt{5}) / 2 \approx 2.62$ & $\mathrm{~F}_{4,1} \times \mathrm{G}_{2,1} \subset \mathrm{E}_{8,1}$ & $\frac{1}{2} A_{4}$ \\
$2+\sqrt{3} \approx 3.73$ & $\mathrm{SU}(2)_{10} \subset \mathrm{Spin}(5)_{1}$ & $E_{6} / / A_{3}$ \\
$2+\sqrt{3} \approx 3.73$ & $\mathcal{B} \subset \operatorname{Spin}(16)_{1}$ & $\left(\mathbb{Z}_{2}+2\right) / / \mathbb{Z}_{2}$ see Example 4.24 \\
$(5+\sqrt{21}) / 2 \approx 4.79$ & $G_{2,3} \subset E_{6,1}$ & $\left(\mathbb{Z}_{3}+3\right) / / \mathbb{Z}_{3}$ \\
$(11+3 \sqrt{13}) / 2 \approx 10.91$ & $? \subset \mathrm{E}_{6,1} \times \mathrm{SU}(3)_{1}$ & $\left(\mathbb{Z}_{3}^{2}+9\right) / /\left(\mathbb{Z}_{3}^{2}\right)$ see Problem 4.27 \\
$5+2 \sqrt{6} \approx 18.80$ & $G_{2,4} \subset \operatorname{Spin}(14)_{1}$ & $F / / \mathbb{Z}_{4}[\mathrm{EP} 15$, Fig. 27/28]
\end{tabular}

TABLE 1. Examples of generalized orbifolds by a hypergroup $K=\left\{c_{0}, c_{1}\right\}$ with $c_{1} c_{1}=d^{-1} \cdot c_{0}+(d-1) d^{-1} \cdot c_{1}$.

One can easily check that the matrix

$$
\left(\chi_{i}^{m}\right)=\left(\begin{array}{cc}
1 & 1 \\
1 & -d^{-1}
\end{array}\right)
$$

gives the characters of the hypergroup, i.e. $c_{i} \mapsto \chi_{i}^{m}$ gives a one-dimensional representation for very $m=0,1$. It follows as in Remark 4.23 that the dual hypergroup $\hat{K}$ can be identified with $K$ and that the matrix (4.6) defines a bicharacter. This fits together with the general theory for commutative finite hypergroups Wil97.

It follows that

$$
\phi_{1}^{I}(a+b)=a-d^{-1} b, \quad a \in \mathcal{A}(I), b \in \mathcal{B}(I) \ominus \mathcal{A}(I) .
$$


Namely, every element $b \in \mathcal{B}(I)$ can be written as $b=a_{0} \psi_{0}+a_{1} \psi_{1}$ with $a \in \mathcal{A}(I)$, where $\psi_{i} \in$ $\operatorname{Hom}\left(\iota, \iota \rho_{i}\right)\left(\psi_{0}=1\right)$ where the dual canonical endomorphism equals $[\theta]=\left[\rho_{0}=\mathrm{id}_{\mathcal{A}(I)}\right] \oplus\left[\rho_{1}\right]$. We have $\phi_{i}^{I}\left(\psi_{m}\right)=\chi_{j}^{m} \psi_{m}$. For a general element we get:

$$
\phi_{i}^{I}(b)=\sum_{m} a_{m} \chi_{j}^{m} \psi_{m}, \quad b=\sum_{m} a_{m} \psi_{m}, \quad a_{m} \in \mathcal{A}(I) .
$$

We will study the general harmonic analysis in a future publication.

We can ask if $K$ comes from a fusion ring $]^{1}$ as characterized in Proposition 2.13. But in general it turns out to be no fusion ring. Let us consider $K=\left\{c_{0}, c_{1}\right\}$ which we rescales with $\tilde{c}_{1}=\lambda \cdot c_{1}$ with $\lambda \in(0, \infty)$, as follows:

$$
\tilde{c}_{1} \tilde{c}_{1}=\frac{\lambda^{2}}{w} \cdot c_{0}+\frac{(w-1) \lambda}{w} \cdot \tilde{c}_{1}
$$

The canonical choice to look like a fusion ring is $\tilde{c}_{1} \tilde{c}_{1}=c_{0}+\mu \cdot \tilde{c}_{1}$, i.e. $\lambda=\sqrt{w}$. The following example shows that $\mu$ is not necessarily an integer.

Example 4.24. Consider the near group fusion ring of the even part of the $E_{6}$ subfactor $F$ (which is $\mathbb{Z}_{2}+2$ in the notation of the following Lemma 4.25 ) and let $K=K_{F} / / \mathbb{Z}_{2}=\left\{c_{0}, c_{1}\right\}$ (cf. Corollary 4.20 , which is realized by conformal nets as follows. Let $\mathcal{B}$ be the net realizing the double of the even part of the $E_{6}$ subfactor from Bis16b which is a $\mathbb{Z}_{2}$-simple current extension of $\mathcal{A}_{\mathrm{SU}(2)_{10}} \otimes \mathcal{A}_{\mathrm{Spin}(11)_{1}}$. This nets embeds into $\mathcal{A}_{\mathrm{E} 8,1}$ :

$$
\mathcal{B} \subset \mathcal{A}_{\mathrm{Spin}(16)_{1}} \subset \mathcal{A}_{\mathrm{E}_{8,1}}, \quad \mathcal{B}:=\left(\mathcal{A}_{\mathrm{SU}(2)_{10}} \otimes \mathcal{A}_{\mathrm{Spin}(11)_{1}}\right) \rtimes \mathbb{Z}_{2} .
$$

Then the inclusions are generalized orbifolds as follows:

$$
\mathcal{A}_{\mathrm{Spin}(16)_{1}}=\mathcal{A}_{E_{8}}^{\mathbb{Z}_{2}}, \quad \mathcal{B}=\mathcal{A}_{E_{8}}^{K_{F}}, \quad \mathcal{B}=\mathcal{A}_{\mathrm{Spin}(16)_{1}}^{K_{F} / / \mathbb{Z}_{2}} .
$$

In this case, we get $\tilde{c}_{1} \tilde{c}_{1}=c_{0}+\sqrt{2} \cdot \tilde{c}_{1}$ which is not integral.

The lattice of intermediate nets of $\mathcal{A}_{\mathrm{SU}(2)_{10}} \otimes \mathcal{A}_{\mathrm{Spin}(11)_{1}} \subset \mathcal{A}_{\mathrm{E}_{8,1}}$ is given as follows:

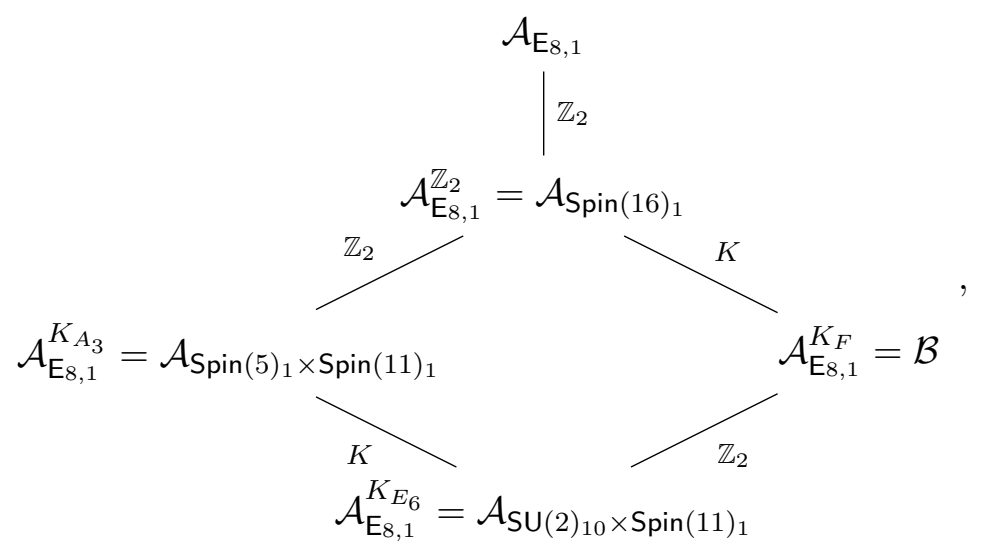

where $K=\left\{c_{0}, c_{1}\right\}$ is the hypergroup from above and $K_{A_{3}}, K_{E_{6}}$ are the hypergroups corresponding to the respective fusion rings.

More general, for quotients of near group fusion rings by the group we get the following normalization.

1 The author thanks V.F.R Jones and D. Penneys for asking such a question. 
Lemma 4.25. Consider the near group fusion ring $F$ of type $G+m$, i.e. $F=G \cup \rho$ with $[\rho]^{2}=$ $\sum_{g \in G}[g]+m[\rho]$. Then the hypergroup $K=K_{F} / / G=\left\{c_{0}, c_{1}\right\}$ is given by

$$
c_{1} c_{1}=\frac{1}{w} \cdot c_{0}+\frac{w-1}{w} \cdot c_{1}, \quad w=\frac{m \sqrt{m^{2}+4 n}+m^{2}+2 n}{2 n},
$$

which can be rescaled to be:

$$
\begin{aligned}
& \tilde{c}_{1} \tilde{c}_{1}=c_{0}+\frac{m}{\sqrt{n}} \cdot \tilde{c}_{1}, \\
& \hat{c}_{1} \hat{c}_{1}=n \cdot c_{0}+m \cdot \hat{c}_{1} .
\end{aligned}
$$

We note that equation (3) is exactly the polynomial whose positive solution is $d \rho$ and that although we do not get a fusion ring we can still get a based ring over $\mathbb{Z}$. It would be interesting if this has a deeper reason when there is a realization by conformal nets. Particularly, $K=\left\{c_{0}, c_{1}\right\}$ is self-dual and the dual hypergroup plays a certain rules for the fusion of charged fields giving the extension.

Similarly, for the Haagerup-Izumi fusion categories, we have:

Lemma 4.26. Consider the Haagerup-Izumi fusion ring $F=G \cup\{g \rho\}_{g \in G}$ with $|G|=n$ and fusion rules $[g][\rho]=\left[g^{-1}\right][\rho],[\rho]^{2}=[e]+\sum_{g \in G}[g \rho]$. Then $K=K_{F} / / G=\left\{c_{0}, c_{1}\right\}$ with:

$$
c_{1} c_{1}=\frac{1}{w} \cdot c_{0}+\frac{w-1}{w} \cdot c_{1}, \quad w=\frac{2+n^{2}+n \sqrt{n^{2}+4}}{2},
$$

which can be rescaled to be:

$$
\tilde{c}_{1} \tilde{c}_{1}=c_{0}+n \cdot \tilde{c}_{1} .
$$

We remark that the fusion rules (4) have categorifications (in terms of fusion categroies) only for $n=0,1$ Ost03], while the Izumi-Haagerup categories are shown to exist for many $n$, including $n=9$ [EG11]. It is important to remark that the "rules" (34) do not have a direct interpretation of fusion rules.

We give the following problem analogue to the construction of the Haagerup VOA proposed in EG11.

Problem 4.27. For $w=(11+3 \sqrt{13}) / 2$, find a proper action of the hypergroup $K=\left\{c_{0}, c_{1}=\right.$ $\left.w^{-1 / 2} \tilde{c}_{1}\right\}$ with $\tilde{c}_{1} \tilde{c}_{1}=c_{0}+3 \tilde{c}_{1}$ on the net $\mathcal{A}_{A_{2} \times E_{6}}$ associated with the even lattice of $A_{2} \times E_{6}$, such that $\operatorname{Rep}\left(\mathcal{A}_{A_{2} \times E_{6}}^{K}\right)$ is braided equivalent to the quantum double of the Haagerup subfactor.

It is basically enough to construct a non-trivial self-adjoint extremal stochastic map $\phi \in \operatorname{Stoch}_{\Omega}(A)$ on $A=\mathcal{A}_{A_{2} \times E_{6}}(I)$ with $\phi \circ \phi=w^{-1} \mathrm{id}_{A}+\left(1-w^{-1}\right) \phi$. which is compatible with the net structure.

\section{Commutative Q-Systems in Unitary Modular Tensor Categories and Inclusions of Completely Rational Conformal Nets}

5.1. Quantum Double Subfactors and Lagrangian Q-systems. In this subsection we want to show that every Lagrangian Q-system comes from a Longo-Rehren subfactor, see Section 2.4. We saw that if a UMTC $\mathcal{C}$ is braided equivalent to $Z(\mathcal{F})$ for a UFC $\mathcal{F}$ then it contains a Lagrangian Q-system, namely $\Theta$ from the Longo-Rehren inclusion. The converse is also true.

Let $\Theta$ be a Lagrangian Q-system in a UMTC $\mathcal{C}={ }_{A} \mathcal{C}_{A} \subset \operatorname{End}(A)$. Consider the category generate by $\alpha^{+}$-induction $\mathcal{D}_{+}={ }_{B} \mathcal{C}_{B}^{+}$, which is equivalent to the category of modules $\mathcal{C}_{\Theta} \cong{ }_{A} \mathcal{C}_{B}$, BKLR16. BKLR15. Let $\rho \in{ }_{A} \mathcal{C}_{A}$, then $\alpha_{\rho}^{+}$has a natural half-braiding $\mathcal{E}_{\rho}$. Therefore it lifts to the center $Z\left({ }_{B} \mathcal{C}_{B}^{+}\right)$. We get an equivalence

$$
{ }_{A} \mathcal{C}_{A} \rightarrow Z\left({ }_{B} \mathcal{C}_{B}^{+}\right): \rho \mapsto\left(\alpha_{\rho}^{+}, \mathcal{E}_{\rho}\right)
$$


and it follows.

Proposition 5.1. Let $\Theta$ be a Lagrangian $Q$-system in a UMTC $\mathcal{C}$. Then $\mathcal{C}$ is braided equivalent to $Z\left(\mathcal{D}_{+}\right)$. In particular, a UMTC $\mathcal{C}$ admits a Lagrangian $Q$-system if and only if $\mathcal{C}$ is braided equivalent to $Z(\mathcal{F})$ for some $U F C \mathcal{F}$.

Proof. We have

$$
Z\left({ }_{B} \mathcal{C}_{B}^{+}\right) \cong{ }_{A} \mathcal{C}_{A} \otimes_{B} \mathcal{C}_{B}^{0}{ }^{\text {rev }} \cong{ }_{A} \mathcal{C}_{A}
$$

thus $\mathcal{F}={ }_{B} \mathcal{C}_{B}^{+}$does the job.

Lemma 5.2. Let $\Theta \in{ }_{A} \mathcal{C}_{A}$ be a Lagrangian $Q$-system and $A \subset B$ be the corresponding subfactor. Then

$$
{ }_{A} \mathcal{C}_{A} \rightarrow Z\left({ }_{B} \mathcal{C}_{B}^{+}\right): \rho \mapsto\left(\alpha_{\rho}^{+}, \mathcal{E}_{\rho}\right)
$$

is a braided equivalence.

Lemma 5.3. Let $\Theta \in{ }_{A} \mathcal{C}_{A} \subset \operatorname{End}(A)$ be a commutative a commutative $Q$-system with corresponding subfactor $A \subset B$. Then $\iota \rho \mapsto \alpha_{\rho}^{ \pm}$extends to an isomorphism of categories:

$$
{ }_{B} \mathcal{C}_{A} \rightarrow{ }_{B} \mathcal{C}_{B}^{ \pm}
$$

Proof. By construction $\alpha$-induction fulfills $\iota \rho=\alpha_{\rho}^{ \pm} \iota$. By [BE98, Lemma 3.5], see [BEK99, p. 454] we have $\operatorname{Hom}(\iota \rho, \iota \sigma)=\operatorname{Hom}\left(\alpha_{\rho}^{ \pm}, \alpha_{\sigma}^{ \pm}\right)$and the statement follows.

The map $\alpha_{\rho}^{+} \mapsto \alpha_{\bar{\rho}}^{-}$extends to an equivalence

$$
\left({ }_{B} \mathcal{C}_{B}^{+}\right)^{\text {op }} \rightarrow{ }_{B} \mathcal{C}_{B}^{-}
$$

of unitary fusion categories.

Proposition 5.4. Let ${ }_{A} \mathcal{C}_{A}$ be an UMTC and $A \subset B$ with corresponding $Q$-system $(\theta, w, x)$ in ${ }_{A} \mathcal{C}_{A}$.

(1) If $\Theta$ is commutative, then ${ }_{M} \mathcal{C}_{M}^{+} \cong\left({ }_{M} \mathcal{C}_{M}^{-}\right)^{\text {op }}$.

(2) If $\Theta$ is Lagrangian, then ${ }_{M} \mathcal{C}_{M} \cong{ }_{M} \mathcal{C}_{M}^{+} \otimes{ }_{M} \mathcal{C}_{M}^{-}$.

Proof. The first property can be directly seen by seeing ${ }_{B} \mathcal{C}_{B}$ as a bimodule category and realizing that the opposite order gives the opposite braiding. For the second statement we can use the relative braiding in [BEK01].

Proposition 5.5. Let $\Theta$ be a Lagrangian Q-system in ${ }_{A} \mathcal{C}_{A}$ with associated subfactor $A \subset B$. Then $\Theta$ is dual to the Longo-Rehren Q-system associated with ${ }_{B} \mathcal{C}_{B}^{+}$, i.e. $A \subset B$ is conjugate to the Longo-Rehren inclusion associated with ${ }_{B} \mathcal{C}_{B}^{+}$.

Proof. We consider $S=A \otimes A^{\text {op }} \subset T=B \otimes B^{\text {op }}$ given by the Lagrangian Q-system $\Theta \otimes \Theta^{\text {op }}$ in ${ }_{S} \mathcal{C}_{S} \cong{ }_{A} \mathcal{C}_{A} \otimes{ }_{A} \mathcal{C}_{A}{ }^{\text {rev }}$. But a Lagrangian Q-system in ${ }_{S} \mathcal{C}_{S} \cong{ }_{A} \mathcal{C}_{A} \bigotimes_{A} \mathcal{C}_{A}{ }^{\text {rev }}$ comes from the $\alpha$-induction construction Reh00], see [BKLR16, Proposition 5.2]. By Kaw02] it follows that $S \subset T$ is dual to the Longo-Rehren inclusion with respect to ${ }_{B} \mathcal{C}_{B}$. By Galois correspondence $A \subset B \cong$ $A \otimes B^{\text {op }} \subset B \otimes B^{\text {op }}$ is also dual to a Longo-Rehren inclusion, namely the one with ${ }_{B} \mathcal{C}_{B}^{+}$.

Corollary 5.6. Let $A \subset B$ with $\Theta=(\theta, x, w)$ a commutative $Q$-system in a UMTC ${ }_{A} \mathcal{C}_{A}$. Then $A \subset B$ is conjugated to $S \subset M$, where $S \subset M \subset T$ and $S \subset T$ is the Longo-Rehren subfactor with respect to ${ }_{B} \mathcal{C}_{B}^{+}$.

Proof. Consider the inclusion $A \otimes B^{\mathrm{op}} \subset B \otimes B^{\mathrm{op}} \subset B_{\mathrm{LR}}$, where $B^{\text {op }} \subset B_{\mathrm{LR}}$ is the Longo-Rehren extension. This gives a Lagrangian Q-system in ${ }_{A} \mathcal{C}_{A} \otimes_{B} \mathcal{C}_{B}^{0}{ }^{\text {rev }}$ and the statement follows from Proposition 5.5 . 
Therefore $A \subset B$ (from Corollary 5.6) can be seen as a generalized group subgroup subfactor, where we replaced (sub-) groups by unitary fusion (sub-) categories and the group action by the Longo-Rehren subfactor.

Remark 5.7. Since the canonical endomorphisms of the Longo-Rehren inclusion has no multiplicities and Corollary 5.6 implies that the canonical endomorphism $\gamma$ of a subfactor $A \subset B$ coming from a commutative Q-system in a UMTC ${ }_{A} \mathcal{C}_{A}$ has no multiplicties. We note that the statement of Proposition 4.1 is stronger, since it only assumes $\theta$ to be a commutative Q-system in any braided rigid $C^{*}$-tensor category.

5.2. Inclusions of Completely Rational Nets and Categorical Restrictions. We have the following simple lemma.

Lemma 5.8 (cf. [BE99, Corollary 3.18]). Let ${ }_{A} \mathcal{C}_{A}$ be a UMTC and $(\theta, w, x)$ a commutative Q-system in ${ }_{A} \mathcal{C}_{A}$ corresponding subfactor $A \subset B$ and dual $Q$-system $\Gamma=(\gamma, v, y)$. Then $[\gamma] \cap{ }_{B} \mathcal{C}_{B}^{0}=[\mathrm{id}]$, i.e. if $\alpha \in{ }_{B} \mathcal{C}_{B}^{0}$ irreducible with $\alpha \prec \gamma$, then $[\alpha]=[\mathrm{id}]$.

Proof. By Frobenius reciprocity we have: $\alpha \prec \gamma \equiv \iota \bar{\iota}$ if and only if $\operatorname{id}_{A} \prec \bar{\iota} \alpha \iota$. Let $\alpha \in{ }_{B} \mathcal{C}_{B}^{0}$, then $[\bar{\iota} \alpha \iota]=\bigoplus_{\rho} b_{\alpha}^{\rho}[\rho]$ with $b_{\alpha}^{\rho}=\langle\rho, \bar{\iota} \alpha \iota\rangle$. But the blockform [BEK00, Equation 16 and Proposition 3.4]

$$
Z_{\rho \sigma}=\sum_{\alpha \in \operatorname{Irr}\left({ }_{B} \mathcal{C}_{B}^{0}\right)} b_{\alpha}^{\rho} b_{\alpha}^{\sigma}, \quad Z=\sum_{\alpha \in \operatorname{Irr}\left({ }_{B} \mathcal{C}_{B}^{0}\right)}\left|\sum_{\rho \in \operatorname{Irr}\left({ }_{A} \mathcal{C}_{A}\right)} b_{\alpha}^{\rho} \chi_{\rho}\right|^{2}
$$

of the modular invariant $Z_{i j}=\left\langle\alpha_{\rho_{i}}^{+}, \alpha_{\rho_{j}}^{-}\right\rangle$and the normalization of the modular invariant $Z_{00}=$ $\left\langle\operatorname{id}_{B}, \operatorname{id}_{B}\right\rangle=1$ (because $B$ is a factor |BKLR15|) implies $b_{\alpha}^{\operatorname{id}_{A}}=\delta_{\alpha, \operatorname{id}_{B}}$.

We have the following interpretation for a local finite index inclusion of completely rational nets $\mathcal{A} \subset \mathcal{B}$. Let $\gamma: \mathcal{B}(I) \rightarrow \mathcal{A}(I) \subset \mathcal{B}(I)$ be the canonical endomorphism. Then it is purely build out of solitonic sectors of $\mathcal{B}$.

Corollary 5.9. Let ${ }_{A} \mathcal{C}_{A}$ be a UMTC and $\Theta$ be a commutative $Q$-system with associated subfactor $A \subset B$. The Jones extension $A \subset B \subset B_{1}=B \vee\left\{e_{A}\right\}$, then $B \subset B_{1}$ comes from a Q-system in ${ }_{B} \mathcal{C}_{B}^{0}$ if and only if $A=B$.

In particular, $A \subset B_{1}$ comes from a commutative $Q$-system in ${ }_{A} \mathcal{C}_{A}$ if and only if $A=B$.

Proof. For the second part we use that local Q-systems $\tilde{\Gamma} \prec \Gamma$ come from commutative Q-systems in $\Gamma \in{ }_{M} \mathcal{C}_{M}^{0}$.

The following corollary shows that Jones basic construction in either of the two directions applied to a non-trivial local inclusions of completely rational nets does not give another net.

Corollary 5.10. Let $\mathcal{A}$ be a completely rational net, $A=\mathcal{A}(I)$ and ${ }_{A} \mathcal{C}_{A}=\operatorname{Rep}{ }^{I}(\mathcal{A})$ and $B=\mathcal{B}(I)$ for $\mathcal{B} \supset \mathcal{A}$ a finite index local extension. Consider the basic construction $A_{1}=\bar{\iota}(A) \subset A \subset B \subset$ $B_{1}=B \vee\left\{e_{A}\right\}$. Then the following are equivalent:

(1) $B_{1}$ comes from a subnet of $\mathcal{A}$,

(2) $B_{1}$ gives a (non-local) extension of $\mathcal{B}$, and

(3) $A=B$, i.e. $\mathcal{A}=\mathcal{B}$.

Proof. $(3) \Rightarrow(1),(2)$ are trivial. Given (2) Corollary 5.9 implies $A=B$. Similarly, if (1) holds then $A_{1}=A$ and therefore $A=B$.

Let $\mathcal{B}$ be a completely rational conformal net, i.e. $\operatorname{Rep}(\mathcal{B})$ is UMTC. The following proposition gives restriction on the possible representation categories $\operatorname{Rep}(\mathcal{A})$ of a finite index subnet $\mathcal{A} \subset \mathcal{B}$. 
We say a functor $K: \mathcal{C} \rightarrow \mathcal{F}$ from a braided UFC $\mathcal{C}$ to a UFC is central, if there is a braided functor $\tilde{K}: \mathcal{C} \rightarrow Z(\mathcal{F})$, such that the following diagram commutes:

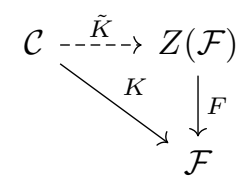

Proposition 5.11. Let $\mathcal{B}$ be a completely rational conformal net. If $\mathcal{A} \subset \mathcal{B}$ is a finite index subnet, then

(1) There is a UFC $\mathcal{F}$ with and a injective functor $\overline{\operatorname{Rep}(\mathcal{B})} \rightarrow \mathcal{F}$, which is central.

(2) $\operatorname{Rep}(\mathcal{A})$ is braided equivalent to $C_{Z(\mathcal{F})}(\overline{\operatorname{Rep}(\mathcal{B})})$.

Proof. Take $\mathcal{F}:={ }_{B} \mathcal{C}_{B}^{+}$, then $Z(\mathcal{F}) \cong{ }_{A} \mathcal{C}_{A} \otimes{ }_{B} \mathcal{C}_{B}^{0 \text { rev }}$ and (2) follows. That ${ }_{B} \mathcal{C}_{B}^{0}{ }^{\text {rev }} \rightarrow{ }_{B} \mathcal{C}_{B}$ is central follows from the arguments as in [BEK01, Corollary 4.8].

We note that this is sufficient for the existence on the level of braided subfactors in the following sense.

Proposition 5.12. Let $\mathcal{F}$ be a UFC and $\mathcal{D}$ be a UMTC. If there is an injective and central functor $K: \overline{\mathcal{D}} \rightarrow \mathcal{F}$, then there is a UFC ${ }_{A} \mathcal{C}_{A}$ and a commutative $Q$-system $\Theta$ with corresponding subfactor $A \subset B$, such that $\mathcal{F} \cong{ }_{B} \mathcal{C}_{B}^{+}$and $\mathcal{D}$ braided equivalent to ${ }_{B} \mathcal{C}_{B}^{0}$.

Proof. We may assume that $\mathcal{F} \subset \operatorname{End}(N)$ and by the Longo-Rehren inclusion, see Section 2.4, we get that $Z(\mathcal{F}) \subset \operatorname{End}(A)$ for some $A$. Let $\mathcal{C}:=C_{Z(\mathcal{F})}(\overline{\mathcal{D}})$. Then $Z(\mathcal{F})$ is braided equivalent to $\mathcal{C} \otimes \overline{\mathcal{D}}$ by Müg03c, Theorem 4.2]. Then the Longo-Rehren constructions associated with $\mathcal{F}$ gives a Lagrangian Q-system $\tilde{\Theta}$ in $\mathcal{C} \otimes \overline{\mathcal{D}}$ and we claim that $\Theta \otimes$ id $:=\tilde{\Theta} \cap \mathcal{C} \otimes$ id does the job. As in Corollary 5.6 we get that $\Theta \otimes$ id correspond to the subcategory $\overline{\mathcal{D}} \subset \mathcal{F}$. If we see $\mathcal{C}={ }_{A} \mathcal{C}_{A}$ as a category of endomorphisms and consider $A \subset B$ corresponding to $\Theta$, we get that ${ }_{B} \mathcal{C}_{B}^{0} \cong \mathcal{D}$ and that $Z\left({ }_{B} \mathcal{C}_{B}^{+}\right) \cong{ }_{A} \mathcal{C}_{A} \otimes{ }_{B} \mathcal{C}_{B}^{0}{ }^{\text {rev }}$.

Now we can do the same construction as in the proof of Proposition 5.11 for ${ }_{B} \mathcal{C}_{B}^{+}$and see that we get isomorphic Lagrangian algebras. Therefore we must have an isomorphism between $\mathcal{F}$ and ${ }_{B} \mathcal{C}_{B}^{+}$which gives a (braided) equivalence between $\mathcal{D}$ and ${ }_{B} \mathcal{C}_{B}^{0}$.

For $K: \overline{\mathcal{D}} \rightarrow \mathcal{F}, K: \mathcal{D} \rightarrow \mathcal{G}$ injective and central functors we define $\mathcal{F} \otimes_{\mathcal{D}} \mathcal{G}$ to be the category of left modules ${ }_{\Theta_{\mathrm{LR}}}(\mathcal{F} \otimes \mathcal{G})$, where $\Theta_{\mathrm{LR}}$ is the canonical Longo-Rehren Q-system in $\mathcal{D}^{\text {rev }} \otimes \mathcal{D} \subset \mathcal{F} \otimes \mathcal{G}$. Up to conventions this is in accordance with the notation in [ENO10, Remark 3.9].

Corollary 5.13. Let ${ }_{A} \mathcal{C}_{A}$ be a UMTC and $\Theta$ be a commmutative Q-system with corresponding subfactor $A \subset B$, then

$$
{ }_{B} \mathcal{C}_{B} \cong{ }_{B} \mathcal{C}_{B}^{+} \quad \underset{B}{\mathcal{C}_{B}^{0} B} \mathcal{C}_{B}^{-}
$$

Proof. As before we consider the inclusion $A \otimes B^{\mathrm{op}} \subset B \otimes B^{\mathrm{op}} \subset B_{\mathrm{LR}}$, and we get with ${ }_{B_{\mathrm{LR}}} \mathcal{C}_{B_{\mathrm{LR}}} \cong$ ${ }_{B} \mathcal{C}_{B}^{+} \otimes{ }_{B} \mathcal{C}_{B}^{-}$, that the dual category ${ }_{B \otimes B^{o} p} \mathcal{C}_{B \otimes B^{\text {op }}}$ is equivalent to ${ }_{B} \mathcal{C}_{B} \otimes_{B} \mathcal{C}_{B}^{0}$ rev . One get that $\left({ }_{B} \mathcal{C}_{B}^{0} \nabla_{B} \mathcal{C}_{B}^{0}{ }^{\text {rev }}\right)_{\hat{\Theta}_{\mathrm{LR}}} \cong{ }_{B} \mathcal{C}_{B}^{0}$ and $\left({ }_{B} \mathcal{C}_{B}^{+} \nabla_{B} \mathcal{C}_{B}^{0}{ }^{\text {rev }}\right)_{\hat{\Theta}_{\mathrm{LR}}} \cong{ }_{B} \mathcal{C}_{B}$, which is the same as ${ }_{\Theta_{\mathrm{LR}}}\left({ }_{B} \mathcal{C}_{B}^{+} \nabla_{B} \mathcal{C}_{B}^{-}\right)$.

This is formalization of the statement Ocn01, Theorem 11.1] which considers only the SU $(2)_{k}$ case. 
Let ${ }_{A} \mathcal{C}_{A}$ be UMTC and $A \subset B$ coming from a commutative Q-system in ${ }_{A} \mathcal{C}_{A}$, then we have the following well-known relations for the global dimensions [BEK01, BE00]:

$$
\begin{array}{rlrl}
\operatorname{Dim}\left({ }_{B} \mathcal{C}_{B}\right) & =\operatorname{Dim}\left({ }_{A} \mathcal{C}_{A}\right), & \operatorname{Dim}\left({ }_{B} \mathcal{C}_{B}^{ \pm}\right) & =[B: A]^{-1} \cdot \operatorname{Dim}\left({ }_{A} \mathcal{C}_{A}\right), \\
\operatorname{Dim}\left({ }_{B} \mathcal{C}_{B}^{0}\right) & =[B: A]^{-2} \cdot \operatorname{Dim}\left({ }_{A} \mathcal{C}_{A}\right), & \operatorname{Dim}\left({ }_{B} \mathcal{C}_{B}\right)=\frac{\operatorname{Dim}\left({ }_{B} \mathcal{C}_{B}^{+}\right) \cdot \operatorname{Dim}\left({ }_{B} \mathcal{C}_{B}^{-}\right)}{\operatorname{Dim}\left({ }_{B} \mathcal{C}_{B}^{0}\right)} \\
\frac{\operatorname{Dim}\left({ }_{B} \mathcal{C}_{B}^{ \pm}\right)}{\operatorname{Dim}\left({ }_{B} \mathcal{C}_{B}^{0}\right)} & =[B: A] . &
\end{array}
$$

Remark 5.14. Even without knowing the existence of the net $\mathcal{A}_{\mathrm{Hg}}$ in Problem 4.27 we have on the level of braided subfactors an inclusion $A_{\mathrm{Hg}} \subset A_{E_{6} A_{2}} \subset A_{E_{8}}$. Now by tensoring we get an inclusion $A_{\mathrm{Hg}} \otimes A_{E_{6} A_{2}} \subset A_{E_{6} A_{2}}$ and it is easy to check $A_{\mathrm{Hg}} \otimes A_{E_{6} A_{2}} \subset A_{E_{8}} \otimes A_{E_{8}}$ gives $\mathcal{F}_{\mathrm{Hg}} \otimes \operatorname{Vect} \mathbb{Z}_{3}$. But there is another extension coming from the Longo-Rehren extension of $A_{E_{6} A_{2}} \otimes A_{E_{6} A_{2}}$ (since $\left.\operatorname{Rep}\left(\mathcal{A}_{E 6 A_{2}}\right) \cong \operatorname{Rep}\left(\mathcal{A}_{E_{6} A_{2}}\right)^{\mathrm{rev}}\right)$. The associated UFC of the inclusion $A=A_{\mathrm{Hg}} \subset B=A_{E_{6} A_{2}}$ has to be a $\mathbb{Z}_{3}^{2}+9$ near group category. Namely, the modular invariant of this inclusion [EG11, (2.4)] is of the form

$$
Z=\sum_{i, j} Z_{i j} \chi_{i} \bar{\chi}_{j}=\left|\chi_{0}+\chi_{1}\right|^{2}+2\left|\chi_{2}\right|^{2}+2\left|\chi_{3}\right|^{2}+2\left|\chi_{4}\right|^{2}+2\left|\chi_{5}\right|^{2}
$$

and ${ }_{B} \mathcal{C}_{B}^{0} \cong \operatorname{Rep}\left(\mathcal{A}_{E_{6} A_{2}}\right)$ which has $\mathbb{Z}_{3}^{2}$-fusion rules. Since $\operatorname{tr}\left(\left(Z_{i j}\right)\right)=10$, using BEK99, Corollary 6.13], we know that ${ }_{B} \mathcal{C}_{B}^{+}$has objects $\mathbb{Z}_{3}^{2} \cup\{\rho\}$ and by calculating the global dimension one can conclude that the only possible fusion rules are $\mathbb{Z}_{3}^{2}+9$. This observation is related to Izu15, Example 12.13].

This implies that $\mathcal{F}_{\mathrm{Hg}} \otimes$ Vect $_{\mathbb{Z}_{3}}$ and the obtained $\mathbb{Z}_{3}^{2}+9$ near group category have the same Drinfel'd center. Therefore we have shown:

Proposition 5.15. $\mathcal{F}_{\mathrm{Hg}} \otimes$ Vect $_{\mathbb{Z}_{3}}$ is Morita equivalent to the (unique by [EG14, Table 4]) $\mathbb{Z}_{3}^{2}+9$ near group category.

5.3. The Structure of Generalized Orbifolds of Completely Rational Nets. Let us assume that $\mathcal{B}$ is a completely rational conformal net, so in particular $\operatorname{Rep}(\mathcal{B})$ is a $\operatorname{UMTC}$. The restriction of the structure of finite index inclusions of completely rational nets $\mathcal{A} \subset \mathcal{B}$ gives us a complete characterization of proper hypergroup actions on $\mathcal{B}$. A different and harder problem is how to construct these actions without explicitly knowing the subnet.

Theorem 5.16. Let $\mathcal{B}$ be a completely rational net and $K$ a hypergroup acting properly on $\mathcal{B}$. Then there is a UFC $\mathcal{F}$ and a central embedding $\operatorname{Rep}(\mathcal{B})^{\mathrm{rev}} \rightarrow \mathcal{F}$, such that $K=K_{\mathcal{F}} / / K_{\mathcal{B}}$ and $\operatorname{Rep}\left(\mathcal{B}^{K}\right)=C_{Z(\mathcal{F})}\left(\operatorname{Rep}(\mathcal{B})^{\mathrm{rev}}\right)$, where $K_{\mathcal{B}}$ is hypergroup associated with the Verlinde fusion ring of $\operatorname{Rep}(\mathcal{B})$.

In particular, if $\mathcal{B}$ is holomorphic, then there exists a unitary fusion category $\mathcal{F}$ with $K=K_{\mathcal{F}}$ and $\operatorname{Rep}\left(\mathcal{B}^{K}\right)=Z(\mathcal{F})$.

Proof. This is the special case $M=A$ of Proposition 5.17 below.

We note that a similar structure of actions of double cosets of fusion rings already appeared in [Xu14, Section 2.11 and Theorem 3.8], but there is no reference to stochastic maps. Further, in the present paper, we are deriving it from an axiomatic notion of an action, whose fixed points are always subnets. Indeed, imposing our axioms such an action is unique. We conjecture that the action in on the charged intertwiners $\left\{\psi_{\rho, i}\right\}$ in Xu14 essentially coincides with $\psi_{\rho, i} \mapsto \phi_{k}\left(\psi_{\rho, i}\right)$.

The interpretation of Theorem 5.16 is that a hypergroup $K$ acting properly of on a holomorphic net $\mathcal{B}$ gives a categorification of $K$. A hypergroup $K$ acting properly of on a completely rational 
net $\mathcal{B}$ gives a an extension of $\operatorname{Rep}(\mathcal{B})^{\text {rev }}$ which is central and "hypergraded" by the hypergroup $K$. We note that the representation of $K$ as a double coset of categorifable fusion rings is in general far from unique.

Proposition 5.17. Let $\mathcal{B}$ completely rational and $K$ a hypergroup acting properly on $\mathcal{B}$. Let $\Theta=$ $(\theta, w, x)$ be an irreducible Q-system in $\operatorname{Rep}^{I}(\mathcal{B})$ corresponding to a subfactor $\iota(B) \subset M$ with $B=$ $\mathcal{B}(I)$ and ${ }_{M} \mathcal{C}_{M}=\left\langle\beta \prec \iota \beta \bar{\iota}: \beta \in \operatorname{Rep}^{I}(\mathcal{B})\right\rangle$ the dual category.

Then there is an extension of $\mathcal{F} \supset{ }_{M} \mathcal{C}_{M}$, such that $K=K_{\mathcal{F}} / / K{ }_{M} \mathcal{C}_{M}$. The construction only depends on the Morita equivalence class of $\Theta$.

Proof. Consider $A \otimes B^{\mathrm{op}} \subset B \otimes B^{\mathrm{op}} \subset B_{2}$ coming from the full center construction of $A \subset M$ as in [BKLR16]. Then [Kaw02] implies that $B \otimes B^{\text {op }} \subset B_{2}$ is a Longo-Rehren inclusion w.r.t. ${ }_{M} \mathcal{C}_{M}$ and $A \otimes B^{\mathrm{op}} \subset B_{2}$ is a Longo-Rehren inclusion with respect to an extension $\mathcal{F}$ of ${ }_{M} \mathcal{C}_{M}$. Then from Example 4.12 and Proposition 4.16 we get that $A \otimes B^{\mathrm{op}} \subset B \otimes B^{\mathrm{op}}$ and therefore $A \subset B$ is a generalized fixed point with hypergroup $K=K_{\mathcal{F}} / / K_{M^{\mathcal{C}}}$.

Let us call $\mathcal{F}$ a $K$-hypergraded extension of $\mathcal{G}$ if $K=K_{\mathcal{F}} / / K_{\mathcal{G}}$. The proposition gives many $K$-graded extensions from inclusions of nets. This is just a categorical result and we get:.

Corollary 5.18. If $\mathcal{D}$ is a UMTC and $\mathcal{F}$ a central extension of $\mathcal{D}$. Let $K=K_{\mathcal{F}} / / K_{\mathcal{D}}$. Then there is a $K$-hypergraded extension for every $U F C \tilde{\mathcal{D}}$ Morita equivalent to $\mathcal{D}$.

More generally, let $\mathcal{F}$ be an extension of $\mathcal{G}$ and $K=K_{\mathcal{F}} / / K_{\mathcal{G}}$. Then we get a $K$-graded extension $\tilde{\mathcal{F}}$ of $\tilde{\mathcal{G}}$ for every Lagrangian $Q$-system $\Theta$ in $Z(\mathcal{G})$, where $\tilde{\mathcal{G}}=Z(\mathcal{G})_{\Theta}$.

Proof. Consider the Longo-Rehren inclusion $S \subset T=M \otimes M^{\text {op }}$ w.r.t. $\mathcal{F} \subset \operatorname{End}(M)$ with ${ }_{S} \mathcal{C}_{S} \cong$ $Z(\mathcal{F})$. Let $S \subset M \subset T$ be the intermediate subfactor associated with $\mathcal{G}$ and $\Theta$ be an Lagrangian Q-system in $Z(\mathcal{G}) \subset{ }_{M} \mathcal{C}_{M}$ with associated subfactor $M \subset T_{\Theta}$. Then $M \subset T_{\Theta}$ is a Longo-Rehren inclusion from $Z(\mathcal{G})_{\Theta} \cong{ }_{\Theta} Z(\mathcal{G})_{\Theta}^{+}$and $S \subset T_{\Theta}$ is Longo-Rehren inclusion coming from an extension of $\tilde{\mathcal{F}}$ of $\tilde{\mathcal{F}}$. Since the hypergroup $K$ can be recovered from $S \subset M$ and $K=K_{\tilde{\mathcal{F}}} / / K_{\tilde{\mathcal{G}}}$ holds by Proposition 4.16 and Example 4.12 , we conclude that $\tilde{\mathcal{F}}$ is a $K$-hypergraded extension of $\tilde{\mathcal{G}}$.

\section{Possible Generalization to Infinite Actions}

We expect that our analysis generalizes to infinite index, were we expect to get semi-compact inclusions. Particularly interesting seem the following inclusions: Let $\mathcal{A}$ be a diffeomorphism covariant net, then there is an irreducible subnet $\operatorname{Vir}_{c} \subset \mathcal{A}$ and the net $\operatorname{Vir}_{c}$ is minimal by [Car98. If $\mathcal{A}$ is completely rational and the central charge $c \geq 1$, then we necessarily have that $\left[\operatorname{Vir}_{c}(I): \mathcal{A}(I)\right]>\infty$. We expect that $\operatorname{Vir}_{c} \subset \mathcal{A}$ might come from a continuous hypergroup. For $c>1$ the net $\operatorname{Vir}_{c}$ is not strongly additive and the inclusion is never quasi-regular (discrete) in the sense of [ILP98. For the case $c=1$, by Reh94, Car04, Xu05, we know that $\operatorname{Vir}_{c=1}=\mathcal{A}_{\mathrm{SU}(2)_{1}}^{\mathrm{SO}(3)}$ is a fixed point by a compact group. We can consider $\mathcal{A}=\mathcal{A}_{E_{7}} \equiv \mathcal{A}_{\mathrm{E}_{7,1}}$, the conformal net associated with the even lattice $E_{7}$, or equivalently cf. $\mid$ Bis12], with the loop group of $E_{7}$ at level 1 . The net $\tilde{\mathcal{A}}$ has the

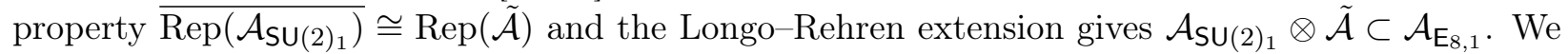
can consider the inclusion

$$
\operatorname{Vir}_{c=1} \otimes \mathcal{A}_{\mathrm{E}_{7,1}} \subset \mathcal{A}_{\mathrm{SU}(2)_{1}} \subset \mathcal{A}_{\mathrm{E} 8,1}
$$

We have that $\operatorname{Vir}_{c=1} \otimes \mathcal{A}_{\mathrm{E}_{7,1}}$ contains a symmetric rigid $\mathrm{C}^{*}$-tensor category $\mathcal{C}$ in the sense of $[\overline{\mathrm{DR}} 89 \mathrm{~b}]$, which is generated by $\operatorname{Rep}(\mathrm{SO}(3))$ and a $d=2$ object with trivial twist and $\operatorname{Rep}(\mathrm{SU}(2))$ fusion rules cf. $\mathrm{Xu05}$, Lemma 4.1]. One can conclude that $\mathcal{C}$ is braided equivalent to $\operatorname{Rep}(\operatorname{SU}(2))$ and that

$\operatorname{Vir}_{c=1} \otimes \mathcal{A}_{\mathrm{E}_{7,1}}=\mathcal{A}_{\mathrm{E}_{8,1}}^{\mathrm{SU}(2)} \subset \mathcal{A}_{\mathrm{E}_{8,1}}$ is an extension by the dual of a compact group in the sense Doplicher-Roberts reconstruction theorem [DR89a, DR90, see also [Xu05]. We get an action of 
the compact group $\mathrm{SU}(2)$ and expect that the inclusion can be seen as a continuous Longo-Rehren inclusion associated with a SU(2)-kernel, i.e. $\alpha: \mathrm{SU}(2) \rightarrow \operatorname{Out}(M)$ for a type $\mathrm{III}_{1}$ factor $M$. We note that this is indeed true for every finite subgroup $G \subset \mathrm{SU}(2)$, where we get a that the module category associated with $\mathcal{A}_{E_{8}}^{G} \subset \mathcal{A}$ is a unitary fusion category equivalent to $\operatorname{Vect}{ }_{G}^{\omega}$ for some $[\omega] \in H^{3}(G, \mathbb{T})$. We also have for $G=\mathbb{Z}_{2}$ that $[\omega]$ is the non-trivial cohomology class, since $\operatorname{Rep}\left(\mathcal{A}_{\mathrm{SU}(2)_{1}} \otimes \mathcal{A}_{E_{7}}\right)$ is braided equivalent to $Z\left(\operatorname{Rep}\left(\mathcal{A}_{\mathrm{SU}(2)_{1}}\right)\right)$ and $\operatorname{Rep}\left(\mathcal{A}_{\mathrm{SU}(2)_{1}}\right) \cong \operatorname{Vect}{ }_{\mathbb{Z}_{2}}^{\omega}$, where $[\omega]=1$ is the non-trivial "generator" in $H^{3}\left(\mathbb{Z}_{2}, \mathbb{T}\right) \cong \mathbb{Z}_{2}$.

Let $\mathcal{B}$ be a diffeomorphism covariant conformal net, i.e. there is is an irreducible subnet $\operatorname{Vir} \subset \mathcal{B}$ Car04, Proposition 3.7], generated by the projective unitary representation of Diff $\left(\mathbb{S}^{1}\right)$. One could define the "quantum automorphism (semi)group" qAut $(\mathcal{B})$ of $\mathcal{B}$ to be the convex space of elements $\phi$, with

- $\phi=\left\{\phi^{I} \in \operatorname{Stoch}_{\Omega}(\mathcal{B})\right\}_{I \in \mathcal{I}}$ is a compatible family,

- $\phi^{I}$ has an $\Omega$ adjoint,

- $\phi^{I}$ is $\operatorname{Vir}(I)$-bimodular.

One gets that $\operatorname{Aut}(\mathcal{B}) \subset \operatorname{qAut}(\mathcal{B})$ and if $\operatorname{Vir} \subset \mathcal{A} \subset \mathcal{B}$, then the family of conditional expectations $E=\left\{E_{I}: \mathcal{B}(I) \rightarrow \mathcal{A}(I) \subset \mathcal{B}(I)\right\}_{I \in \mathcal{I}}$ is contained in qAut $(\mathcal{B})$. Every element in $\phi \in \operatorname{qAut}(\mathcal{B})$ gives an intermediate net $\mathcal{B}^{\phi}(I):=\mathcal{B}(I)^{\phi^{I}}$. A proper finite hypergroup action corresponds to a finite simplex inside $q A u t(\mathcal{B})$. It might be enough to consider $\mathrm{qAut}_{\text {ext }}(\mathcal{B}) \subset \mathrm{qAut}_{0}(\mathcal{B})$ the set of all these maps which are extremal. From $\operatorname{Car04}, \mathrm{Xu05}$ follows that qAut ${ }_{\text {ext }}\left(\mathcal{A}_{\mathrm{SU}(2), 1}\right)=\operatorname{Aut}\left(\mathcal{A}_{\mathrm{SU}(2), 1}\right) \cong \mathrm{SO}(3)$. For $\mathcal{A} \supset \operatorname{Vir}_{c}$ with central charge $c<1$, we have that $\operatorname{Vir}_{c}$ is completely rational and we get that qAut $_{\text {ext }}(\mathcal{A})$ is finite cf. KL04. But for a completely rational net with $c>1$ everything is open.

\section{Appendix A. Completely Positive Maps}

Let $A$ and $B$ be unital $\mathrm{C}^{*}$-algebras. We typically consider $A=B$ and both to be von Neumann algebras. By a map $\phi: A \rightarrow B$ we always mean a linear map. A map $\phi: A \rightarrow B$ is called positive if $\phi(a) \geq 0$ for all $a \geq 0$. Let $n \in \mathbb{N}$. We call $\phi n$-positive if $\phi \otimes \mathrm{id}: A \otimes M_{n}(\mathbb{C}) \rightarrow B \otimes M_{n}(\mathbb{C})$ is positive and completely positive $(\mathrm{CP})$ if it is $n$-positive for every $n \in \mathbb{N}$. A positive map $\phi: A \rightarrow B$ is automatically hermitian, i.e. $\phi\left(a^{*}\right)=\phi(a)^{*}$ for all $a \in A$. We call a map $\phi: A \rightarrow B$ unital if $\phi(1)=1$.

Let $A$ be a $\mathrm{C}^{*}$-algebra and $\phi: A \rightarrow \mathrm{B}(\mathcal{H})$ be linear map. Then $\phi$ is completely positive if and only if there is a representation $\pi: A \rightarrow \mathrm{B}(\mathcal{K})$ and a bounded operator $V \in \mathrm{B}(\mathcal{H}, \mathcal{K})$, such that $\phi(\cdot)=V^{*} \pi(\cdot) V$. In this situation $(\pi, V, \mathcal{K})$ is called a Stinespring dilation. It is called minimal if $\pi(A) V \mathcal{H}$ is dense in $\mathcal{K}$. The minimal Stinespring dilation is unique up to a unitary equivalence. If $V$ is an isometry, then $V^{*} \pi(\cdot) V$ is a unital completely positive map.

If $\phi: A \rightarrow B$ is a unital completely positive map between $\mathrm{C}^{*}$-algebras, we have the KadisonSchwarz inequality $\operatorname{Kad52}$ :

$$
\phi\left(a^{*} a\right) \geq \phi(a)^{*} \phi(a) .
$$

Theorem A.1 ([Cho74]). If $\phi: A \rightarrow B$ is a unital 2-positive map between $C^{*}$-algebras, then $\phi\left(a^{*} a\right)=\phi\left(a^{*}\right) \phi(a)$ if and only if

$$
\phi(x a)=\phi(x) \phi(a), \quad \phi\left(a^{*} x\right)=\phi\left(a^{*}\right) \phi(x),
$$

for all $x \in A$.

Definition A.2. A completely positive map $\phi: A \rightarrow B$ is said to be extremal if every completely positive map with $\psi: A \rightarrow B$ with $\phi-\psi$ completely positive, is a scalar multiple of $\phi$.

Therefore a unital completely positive map is extremal if and only if it cannot be written as a non-trivial convex combination of two unital completely positive maps. 
A.1. Stochastic Maps. A pair $(M, \varphi)$ of a von Neumann algebra $M$ and a faithful normal state $\varphi$ is called a (non-commutative) probability space. Let $\left(M_{i}, \varphi_{i}\right)$ with $i=1,2$ be two probalility spaces. A normal unital completely positive map $\phi: M_{1} \rightarrow M_{2}$ is called a stochastic map from $\left(M_{1}, \varphi_{1}\right)$ to $\left(M_{2}, \varphi_{2}\right)$ provided $\varphi_{2} \circ \phi=\varphi_{1}$. It is called a determistic map if $\phi$ is a $*$-homomorphism. Let $\left(\pi_{i}, \mathcal{H}_{i}, \Omega_{i}\right)$ be the GNS construction of $\left(M_{i}, \varphi_{i}\right)$. By abuse of notation we denote by $\phi: \pi_{1}\left(M_{1}\right) \rightarrow$ $\pi_{2}\left(M_{2}\right)$ the map satisfying $\phi\left(\pi_{1}(m)\right)=\pi_{2}(\phi(m))$ for all $m \in M_{1}$.

A $\left(\varphi_{1}, \varphi_{2}\right)$-adjoint of $\phi$ is a stochastic map $\phi^{\sharp}:\left(M_{2}, \varphi_{1}\right) \rightarrow\left(M_{1}, \varphi_{1}\right)$, such that $\varphi_{2}\left(m_{2} \phi\left(m_{1}\right)\right)=$ $\varphi_{1}\left(\phi^{\sharp}\left(m_{2}\right) m_{1}\right)$ for all $m_{i} \in M_{i}$. Let $\sigma_{t}^{\varphi_{i}}=\operatorname{Ad} \Delta_{\left(M_{i}, \Omega_{i}\right)}^{\mathrm{i} t}$ the modular flow and $J_{i}=J_{\left(M_{i}, \Omega_{i}\right)}$ the modular conjugation. By the Kadison-Schwarz inequality (5) we get a linear contraction $U_{\phi}: \mathcal{H}_{1} \rightarrow$ $\mathcal{H}_{2}$ defined as the closure of $m \Omega_{1} \mapsto \phi(m) \Omega_{2}$ for $m \in M_{1}$. Note that $U_{\phi^{\sharp}}=U_{\phi}^{*}$. The following are equivalent AC82, Proposition 6.1], see also [NSZ03]:

(1) $\phi$ admits $\left(\varphi_{1}, \varphi_{2}\right)$-adjoint $\phi^{\sharp}$.

(2) $\phi \circ \sigma_{t}^{\varphi_{1}}=\sigma_{t}^{\varphi_{2}} \circ \phi$

(3) $J_{\varphi_{2}} U_{\phi}=U_{\phi} J_{\varphi_{1}}$

and in this case we also call $\phi$ a $\left(\varphi_{1}, \varphi_{2}\right)$-preserving Markov map.

We are interested in the case if the non-commutative probability spaces are equal and in standard form $(M, \Omega)$, where $M \subset \mathrm{B}(\mathcal{H})$ and $\Omega \in \mathcal{H}$ a cyclic and separating vector and faithful normal state $\varphi=(\Omega, \Omega)$. Let us denote the modular flow by $\sigma_{t}=\operatorname{Ad} \Delta^{\mathrm{it}}$ and the modular conjugation by $J$. Then a stochastic (endo-) map $\phi:(M, \Omega) \rightarrow(M, \Omega)$ fulfills $(\Omega, \phi(m) \Omega)=(\Omega, m \Omega)$ and a $\Omega$ preserving Markov (endo-) map is such a stoachastic map having an adjoint, and therefore fulfills $\phi \circ \sigma_{t}=\sigma_{t} \circ \phi$ and $U_{\phi} J=J U_{\phi}$.

If $\phi: N \rightarrow N$ is a stochastic map, we can consider the fixed point $N^{\phi}=\{n \in n: \phi(n)=n\}$. The following proposition is a well-known (cf. e.g. AGG02 ) consequence of Choi's Theorem A.1.

Proposition A.3. Let $(N, \varphi)$ be a probability space, i.e. a von Neumann algebra $N$ and faithful state $\varphi$. Let $\phi: N \rightarrow N$ be a $\varphi$-preserving stochastic map, i.e. a normal unital completely positive $\varphi$-preserving map, then $N^{\phi}=\{n \in N: \phi(n)=n\}$ is a von Neumann algebra. If $E=\phi$ is an idempotent, i.e. $E^{2}=E$, then $E(N)$ is a von Neumann algebra and $E$ is the conditional expectation onto its image.

Proof. By the Kadison-Schwarz inequality (5) we have $y:=\phi\left(x^{*} x\right)-\phi\left(x^{*}\right) \phi(x) \geq 0$ is positive for all $x \in N$. Let $x \in N^{\phi}$, i.e. $x=\phi(x)$, then

$$
\varphi(y)=\varphi\left(\phi\left(x^{*} x\right)-\phi(x)^{*} \phi(x)\right)=\varphi\left(x^{*} x\right)-\varphi\left(x^{*} x\right)=0
$$

and since $y$ is positive and $\varphi$ faithful $y \equiv \phi\left(x^{*} x\right)-\phi(x) \phi\left(x^{*}\right)=0$. But then Theorem A.1 implies that $N^{\phi}$ is an algebra and by normality it is a von Neumann algebra.

If $E:=\phi$ is an idempotent, then $E(N)=N^{E}$ and $E$ is a conditional expectation onto its image.

A.2. Connes-Stinespring Construction for Stochastic Maps. Let $\left(M_{i}, \Omega_{i}\right)$ be probability spaces on $\mathcal{H}_{i}$ and $\phi: M_{1} \rightarrow M_{2}$ a stochastic map with $\left(\Omega_{2}, \phi(\cdot) \Omega_{2}\right)=\left(\Omega_{1}, \cdot \Omega_{1}\right)$.

The following construction is the Connes correspondence associated with a UCP map [Con94]. Let $\mathcal{H}_{\phi}$ be the separation and completion of $M_{1} \otimes_{\text {alg }} \mathcal{H}_{2}$ with inner product

$$
(m \otimes \xi, n \otimes \eta)_{\phi}=\left(\xi, \phi\left(m^{*} n\right) \eta\right) .
$$

We get a $M_{1}-M_{2}$ correspondence:

$$
\begin{aligned}
m_{1} \cdot[m \otimes \xi] . m_{2} & :=m_{1} m \otimes \xi . m_{2}, & & \Omega_{\phi}:=\left[1 \otimes \Omega_{2}\right], \\
\left(\Omega_{\phi}, m_{1} \Omega_{\phi}\right)_{\phi} & =\left(\Omega_{1}, m_{1} \Omega_{1}\right), & & m_{1} \in M_{1}, \\
\left(\Omega_{\phi}, \Omega_{\phi} m_{2}\right)_{\phi} & =\left(\Omega_{2}, \Omega_{2} m_{2}\right), & & m_{2} \in M_{2} .
\end{aligned}
$$


We get an isometry $V: \mathcal{H}_{2} \rightarrow \mathcal{H}_{\phi}$ defined by $V \Omega_{2} m_{2}=\left[1 \otimes \Omega m_{2}\right] \equiv \Omega_{\phi} \cdot m_{2}$. Then $\phi(m)=$ $V^{*} \pi_{\phi}(m) V$, where

$$
\pi_{\phi}(m) \xi=m . \xi
$$

Let $M_{1} \subset M_{2}$ be type III factors on a separable Hilbert space $\mathcal{H}$ and $\Omega \in \mathcal{H}$ cyclic and separating for $M_{1}$ and $M_{2}$. Then there is a $U_{2}: \mathcal{H}_{\phi} \rightarrow \mathcal{H}_{2}$ intertwining the right actions of $M_{2}$. Let $\rho=$ $\operatorname{Ad} U_{2} \circ \pi_{\phi}: M_{1} \rightarrow M_{2}$ and $v=U_{2} V \in M_{2}$, then

$$
\phi(m)=V^{*} \pi_{\phi}(m) V=v \rho(m) v .
$$

So we get a pair $(v, \rho)$, such that $\varphi_{2}\left(v^{*} \rho(\cdot) v\right)=\varphi_{1}(\cdot)$. For completely positive maps, we write $\psi \leq \phi$ if $\phi-\psi$ is completely positive. The following is well-known, see also [ILP98, Proposition 2.9]

Lemma A.4. Let $M$ be a type III factor on a separable Hilbert space and $\Omega$ cyclic and separating. Let $\phi, \psi \in \operatorname{Stoch}_{\Omega}(M)$ with $\lambda \psi \leq \phi$ for some $\lambda \in(0,1]$, and let $\phi=v^{*} \rho(\cdot) v$ and $\phi=w^{*} \sigma(\cdot) w$ be the minimal Stinespring representation with $v, w \in M$ and $\rho, \sigma \in \operatorname{End}(M)$. Then there is a contraction $T \in \operatorname{Hom}(\rho, \sigma)$ with $T v=\sqrt{\lambda} \cdot w$.

Proof. Since $\phi-\lambda \psi$ is completely positive we have

$$
\lambda\left\|\sum_{i} \sigma\left(m_{i}\right) w n_{i} \Omega\right\|^{2}=\sum_{i j}\left(n_{i} \Omega, \psi\left(m_{i}^{*} m_{j}\right) n_{j} \Omega\right) \leq \sum_{i j}\left(n_{i} \Omega, \phi\left(m_{i}^{*} m_{j}\right) n_{j} \Omega\right)=\left\|\sum_{i} \rho\left(m_{i}\right) v n_{i} \Omega\right\|^{2}
$$

and get a contraction $T$ defined by $T \rho(m) v n \Omega=\sqrt{\lambda} n \sigma(m) w n \Omega$. For $m=1$ it follows that $T v=\sqrt{\lambda} w$ and therefore $T \rho(m)=\sigma(m) T$.

The next can be seen as a Radon-Nikodym theorem. It basically follows from Pas73, 5.4 Proposition].

Proposition A.5. Let $M$ be a type III factor on a separable Hilbert space and $\Omega$ cyclic and separating. Let $\phi \in \operatorname{Stoch}_{\Omega}(M)$ with minimal Stinespring representation $\phi(\cdot)=v^{*} \rho(\cdot) v$ with $v \in M$ and $\rho \in \operatorname{End}(M)$.

The linear map $T \mapsto \phi_{T}(\cdot)=v^{*} \sigma(\cdot) T v$ is an order preserving bijection between:

- $\left\{T \in \rho(M)^{\prime} \cap M: 0 \leq T \leq 1\right\}$ and

- the set of normal completely positive maps $\psi: M \rightarrow M$ with $\psi(1)=\lambda \cdot 1$ and $\lambda \cdot(\Omega, m \Omega)=$ $(\Omega, \psi(m) \Omega)$ for all $m \in M$.

In particular, $\phi$ is extremal in $\operatorname{Stoch}_{\Omega}(M)$ if and only if $\rho$ is irreducible.

Proof. We first show injectivity. Assume that $\phi_{T}=0$, then

$$
(\rho(a) v \xi, T \rho(b) v \eta)=\left(\xi, v^{*} \rho\left(a^{*} b\right) T v \eta\right)=\left(\xi, \phi_{T}\left(a^{*} b\right) \eta\right)=0
$$

and then $T=0$ because the Stinespring representation is minimal.

We claim that for $0<T \leq 1$ we have that $\phi_{T}$ is (up to scale) state-preserving. If $\phi_{T}(1)=$ $(v \Omega, T v \Omega)=\lambda>0$, then with $\omega(\cdot)=(\Omega, \cdot \Omega)$

$$
\omega(m)=\omega \circ \phi(m)=\lambda \omega_{T}(m)+(1-\lambda) \omega_{1-T}(m), \quad \omega_{S}(m):=(v \Omega, S \rho(m) v \Omega) /(v \Omega, S v \Omega) .
$$

We can extend to states on the $\mathrm{C}^{*}$-algebra $M \otimes_{\min } M^{\prime}$ by $\tilde{\omega}_{\bullet}\left(m_{1} \otimes m_{2}\right)=\left(\Omega \phi_{\bullet}\left(m_{1}\right) m_{2} \Omega\right) /\left(\Omega, \phi_{\bullet}(1) \Omega\right)$ and get $\tilde{\omega}=\lambda \tilde{\omega}_{T}+(1-\lambda) \tilde{\omega}_{T-1}$. But since $\tilde{\omega}=\tilde{\omega}_{1}$ is a pure state we get: $\omega_{T}=\omega_{1-T}=\omega$. 


\section{Appendix B. Tensor Categories}

We give some results on braided tensor categories. We refer to [EGNO15 for a textbook. Most of the statements here are in DMNO13, DNO13. Some statements are implicitly contained and we sketch a proof.

A fusion category $\mathcal{F}$ over a field $\mathbb{K}$ is a $\mathbb{K}$-linear semisimple rigid tensor category with finitely many isomorphism classes of simple objects and finite dimensional spaces of morphisms, such that the unit object 1 is simple. Every fusion category contains a trivial subcategory consisting of multiples of 1 which we denote Vect. We denote the Grothendieck ring of $\mathcal{F}$ by $K(\mathcal{F})$. It is a fusion ring.

Let $\mathcal{C}$ be a non-degenerate braided fusion category. Non-degenerated means that the centralizer $\mathcal{C}^{\prime}=C_{\mathcal{C}}(\mathcal{C})$ is trivial, i.e. $\mathcal{C}^{\prime} \cong$ Vect. Let $A$ be an étale algebra in $\mathcal{C}$, i.e. a commutative and separable algebra. It is called connected if $\operatorname{dim} \operatorname{Hom}(1, A)=1$. We denote by $\mathcal{C}_{A}$ the category of right $A$-modules. If $A$ is a connected étale algebra, then $\mathcal{C}_{A}$ is a fusion category. We denote by $\mathrm{FPdim} X, X \in \mathcal{C}$ the Perron-Frobenius dimension of the object and by $\mathrm{FPdim} \mathcal{C}:=\sum_{X \in \operatorname{Irr}(\mathcal{C})}(\mathrm{FP} \operatorname{dim} X)^{2}$. One has $\mathrm{FPdim} \mathcal{C}_{A}=\mathrm{FPdim} \mathcal{C} / \mathrm{FPdim} A$ DMNO13, Lemma 3.11]. The Drinfel'd center $Z\left(\mathcal{C}_{A}\right)$ is braided equivalent DMNO13, Corollary 3.30] to $\mathcal{C} \otimes \mathcal{C}_{A}^{0 \text { rev }}$, where $\mathcal{C}_{A}^{0}$ is the category of dyslexic modules, which is a non-degenerately braided fusion category. One has $\mathrm{FPdim} \mathcal{C}_{A}^{0}=\mathrm{FPdim} \mathcal{C} /(\mathrm{FP} \operatorname{dim} A)^{2}$ [DMNO13, Corollary 3.32]. A connected étale algebra $A$ in $\mathcal{C}$ is called Lagrangian if $\mathrm{FPdim} A=\sqrt{\mathrm{FPdim} \mathcal{C}}$ and this implies $\mathcal{C}_{A}^{0} \cong$ Vect.

There is a Lagrangian algebra $A$ in $\mathcal{C}$ if and only if $\mathcal{C}$ is braided equivalent to the Drinfel'd center $Z(\mathcal{F})$ for a fusion category $\mathcal{F}$. Namely, if $A$ is Lagrangian we have $\mathcal{C} \cong Z\left(\mathcal{C}_{A}\right)$. Conversely define $A=I(1)$, where $I: \mathcal{F} \rightarrow Z(\mathcal{F})$ is the adjoint of the forgetful functor $F: Z(\mathcal{F}) \rightarrow \mathcal{F}$. Under this identification $A=I(1)$. If $A$ is Lagrangian, then ${ }_{A} \mathcal{C}_{A} \cong \mathcal{C}_{A} \otimes \mathcal{C}_{A}^{\text {op }}$ [DMNO13, Corollary 4.1].

If $\mathcal{F}$ is a fusion category, let us denote the Lagrangian algebra $I(1)$ by $A_{\mathcal{F}}=I(1) \in Z(\mathcal{F})$. For $\mathcal{G} \subset \mathcal{F}$ we get an étale algebra $A_{\mathcal{F} / / \mathcal{G}} \subset A_{\mathcal{F}}$ with $Z(\mathcal{F})_{A_{\mathcal{F} / / \mathcal{G}}}^{0} \cong Z(\mathcal{G})$ given by the order-reversing isomorphism of lattices [DMNO13, Theorem 4.10]. It also follows that $A_{\mathcal{F}}$ is the "composition" of $A_{\mathcal{G}}$ with $A_{\mathcal{F} / / \mathcal{G}}$. By this we mean, that $A_{\mathcal{F} / / \mathcal{G}} \in \mathcal{C}_{A_{\mathcal{G}}}^{0}$ is the algebra $\mathcal{A}_{\mathcal{F}} \supset \mathcal{A}_{\mathcal{G}}$ in $\mathcal{C}$. Further, every Lagrangian algebra $A$ is of the form $A_{\mathcal{F}}$.

Let $\mathcal{F}$ be a fusion category, then there is a bijection between the isomorphism classes of Lagrangian algebras in $Z(\mathcal{F})$ and equivalence classes of indecomposeable $\mathcal{F}$-module categories DMNO13, Proposition 4.8$]$.

Let $\mathcal{C}=Z(\mathcal{F})$ and $A=A_{\mathcal{F}}$. We have that ${ }_{A} \mathcal{C}_{A} \cong \mathcal{F} \otimes \mathcal{F}^{\text {op }}$ [DMNO13, Corollary 4.1] and there is a dual algebra (see [EGNO15, Section 7.12]) $B \in \mathcal{D}={ }_{A} \mathcal{C}_{A}$, such ${ }_{B} \mathcal{C}_{B} \cong \mathcal{C}$. It follows from EGNO15, Propostion 7.13.8, Lemma 8.12.2] that $B=\bigoplus_{X \in \operatorname{Irr}(\mathcal{F})} X \otimes X^{*}$ is the canonical algebra, see also [Müg03b].

Proposition B.1. Let $A$ be a connected étale algebra in a non-degenerately braided fusion category $\mathcal{C}$. Then the inclusion $C_{A}^{0}{ }^{\text {rev }} \rightarrow C_{A}$ is a central functor.

Conversely, if $\mathcal{D}$ is non-degenerately braided fusion category and $\mathcal{F}$ a fusion category. If there is a central injective (fully faithful) functor $\kappa: \mathcal{D}^{\text {rev }} \rightarrow \mathcal{F}$, then there is a non-degenerately braided fusion category $\mathcal{C}$ and a connected étale algebra $A \in \mathcal{C}$, such that $\mathcal{F} \cong \mathcal{C}_{A}$ and $\mathcal{D}=\mathcal{C}_{A}^{0}$. In this case, $\mathcal{C}$ is given as $C_{Z(\mathcal{F})}\left(\kappa\left(\mathcal{D}^{\text {rev }}\right)\right)$.

Proof. We have $Z\left(\mathcal{C}_{A}\right)=\mathcal{C} \otimes \mathcal{C}_{A}^{0}{ }^{\text {rev }}$ and the first statement follows from [DNO13, Example 3.11].

Conversely, take $\mathcal{C}=C_{Z(\mathcal{F})}\left(\mathcal{D}^{\text {rev }}\right)$. Then $Z(\mathcal{F}) \cong \mathcal{C} \otimes \mathcal{D}^{\text {rev }}$ and we get a Lagrangian algebra $A_{\mathcal{F}}$ in $Z(\mathcal{F})$. We get an étale $A \in \mathcal{C}$ with $A \otimes 1=A_{\mathcal{F}} \cap(\mathcal{C} \otimes 1)$ and again from DNO13, Example 3.11] follows $\mathcal{C}_{A}^{0} \cong \mathcal{D}^{\text {rev }}$. Since $\mathcal{D}^{\text {rev }} \rightarrow \mathcal{F}$ is a injective (central) functor $\mathcal{C} \rightarrow \mathcal{F}$ is a surjective (central) functor [DNO13, Theorem 3.12]. Let $\tilde{I}: \mathcal{F} \rightarrow Z(\mathcal{F})$ be the induction functor. Then $\tilde{I}(1)$ is a connected étale algebra in $\mathcal{C}$ isomorphic to $A$ and $\mathcal{F} \cong \mathcal{C}_{A}$ by [DNO13, Section 2.4]. 
Let $\mathcal{D}$ be non-degenerately braided, and assume we have two fusion categories $\mathcal{F}, \mathcal{G}$ with braided central injective functors $\mathcal{D}^{\text {rev }} \rightarrow \mathcal{F}$ and $\mathcal{D} \rightarrow \mathcal{G}$. let us define $\mathcal{F} \otimes_{\mathcal{D}} \mathcal{G}$ by

$$
\mathcal{F} \otimes_{\mathcal{D}} \mathcal{G}={ }_{R}(\mathcal{F} \otimes \mathcal{G})
$$

where $R \in \mathcal{D}^{\text {rev }} \otimes \mathcal{D}$ is the dual algebra to $A_{\mathcal{D}^{\text {rev }}} \in Z(\mathcal{D})$.

The following reflects Ocn01, Theorem 11], in the sense that ${ }_{A} \mathcal{C}_{A}$ (called subgroup) is a fibered product of $\mathcal{C}_{A}$ (corresponding the chiral left part) with $\mathcal{C}_{A}^{\mathrm{op}}\left(\cong{ }_{A} \mathcal{C}\right.$ corresponding to the chiral right part) over $\mathcal{C}_{A}^{0}$ (called the ambichiral part).

Proposition B.2. Assume $A$ is a connected étale algebra in a non-degenerately braided fusion category $\mathcal{C}$, then ${ }_{A} \mathcal{C}_{A} \cong \mathcal{C}_{A} \nabla_{\mathcal{C}_{A}^{0}} \mathcal{C}_{A}^{\mathrm{op}}$.

Proof. We only sketch the proof.

We have $Z\left(\mathcal{C}_{A}\right)=\mathcal{C} \otimes\left(\mathcal{C}_{A}^{0}\right)^{\text {rev }}$. The algebras $A \otimes 1 \subset A_{\mathcal{C}_{A}}$ give the Morita equivalence between $\mathcal{C} \otimes\left(\mathcal{C}_{A}^{0}\right)^{\text {rev }}$ or ${ }_{A} \mathcal{C}_{A} \otimes\left(\mathcal{C}_{A}^{0}\right)^{\mathrm{rev}}$, respectively, and $\mathcal{C}_{A} \otimes \mathcal{C}_{A}^{\text {op }}$. The algebra $A_{\mathcal{C}_{A}}$ is $A \otimes 1$ composed with $A_{\mathcal{C}_{A}^{0}}$. Let $R$ be the dual algebra of $A_{\mathcal{C}_{A}^{0}}$. Then we get ${ }_{R}\left(\mathcal{C}_{A} \otimes \mathcal{C}_{A}^{\text {op }}\right)_{R} \cong{ }_{A} \mathcal{C}_{A} \otimes\left(\mathcal{C}_{A}^{0}\right)^{\text {rev }}$. This restricts to ${ }_{R}\left(\mathcal{C}_{A}^{0} \otimes\left(\mathcal{C}_{A}^{0}\right)^{\text {op }}\right)_{R} \cong \mathcal{C}_{A}^{0} \otimes\left(\mathcal{C}_{A}^{0}\right)^{\text {rev }}$ and ${ }_{R}\left(\mathcal{C}_{A}^{0} \otimes\left(\mathcal{C}_{A}^{0}\right)^{\text {op }}\right) \cong \mathcal{C}_{A}^{0}$. We conclude that $\left(\mathcal{C}_{A} \otimes \mathcal{C}_{A}^{\mathrm{op}}\right)_{R} \cong{ }_{A} \mathcal{C}_{A}$.

Let us denote by ${ }_{A} \mathcal{C}_{A}^{+}$the image of $\mathcal{C}_{A} \otimes 1 \rightarrow{ }_{A} \mathcal{C}_{A}$ and by ${ }_{A} \mathcal{C}_{A}^{-}$the image of $1 \otimes \mathcal{C}_{A} \rightarrow{ }_{A} \mathcal{C}_{A}$. It is easy to see that both inclusions restrict to an equivalence $\mathcal{C}_{A}^{0} \rightarrow{ }_{A} \mathcal{C}_{A}^{0}$, where ${ }_{A} \mathcal{C}_{A}^{0}={ }_{A} \mathcal{C}_{A}^{+} \cap{ }_{A} \mathcal{C}_{A}^{-}$. In this sense one can see ${ }_{A} \mathcal{C}_{A}$ also see as a "fibred product"

$$
{ }_{A} \mathcal{C}_{A}={ }_{A} \mathcal{C}_{A}^{+} \otimes{ }_{A} \mathcal{C}_{A}^{0} \quad{ }_{A} \mathcal{C}_{A}^{-} .
$$

We get a hypergroup $K=K(\mathcal{F}) / / K(\mathcal{G})$ and we say that $\mathcal{F}$ is a $K$-hypergraded extension of $\mathcal{G}$. This generalizes the concept of $G$-graded extensions for a finite group $G$.

Remark B.3. Let $\mathcal{F}$ be a $K$-graded extension of $\mathcal{G}$. For every indecomposable $\mathcal{G}$-module category we get $\tilde{\mathcal{G}}$ Morita equivalent to $\mathcal{G}$ and and an extension $\tilde{\mathcal{F}}$ of $\tilde{\mathcal{G}}$, such that $\tilde{\mathcal{F}}$ is Morita equivalent to $\mathcal{F}$ and $A_{\tilde{\mathcal{F}} / / \tilde{\mathcal{G}}} \cong A_{\mathcal{F} / / \mathcal{G}}$ in $Z(\mathcal{F})=Z(\tilde{\mathcal{F}})$. Namely, Lagrangian algebras over $A_{\mathcal{F} / / \mathcal{G}}$ are in bijection with Lagrangian algebras in $Z(\mathcal{F})_{A_{\mathcal{F} / / \mathcal{G}}}^{0} \cong Z(\mathcal{G})$ cf. [DMNO13, Proposition 3.16] which are in bijection with indecomposable $\mathcal{G}$ module categories [DMNO13, Proposition 4.8]. Given such a Lagrangian algebra $\tilde{A}$, we take $\tilde{\mathcal{F}}=Z(\mathcal{F})_{\tilde{A}}$. We conjecture that $\tilde{\mathcal{F}}$ is a $K$-graded extension of $\tilde{\mathcal{G}}$. This is true in the unitary case by Corollary 5.18 .

The obtained Lagrangian algebra $\tilde{A} \in Z(\mathcal{F})$ with $A_{\mathcal{F} / / \mathcal{G}} \subset \tilde{A}$ is a composition of $A_{\mathcal{F} / / \mathcal{G}}$ and $A_{\tilde{\mathcal{G}}}$. Further, $\tilde{A}=A_{\tilde{\mathcal{F}}}$ for a fusion category $\tilde{\mathcal{F}}$ Morita equivalent to $\mathcal{F}$ and $\tilde{\mathcal{G}} \subset \tilde{\mathcal{F}}$ is the subcategory associated with the subalgebra $A_{\mathcal{F} / / \mathcal{G}} \subset \tilde{A}$. To show that $K(\mathcal{F}) / / K(\mathcal{G}) \cong K(\tilde{\mathcal{F}}) / / K(\tilde{\mathcal{G}})$, it is enough to show that the hypergrading just depends on the dual algebra of $A_{\mathcal{F} / / \mathcal{G}}=A_{\tilde{\mathcal{F}} / / \mathcal{G}}$, for which we just have a proof in the unitary case.

\section{REFERENCES}

[AC82] L. Accardi and C. Cecchini, Conditional expectations in von Neumann algebras and a theorem of Takesaki, J. Funct. Anal. 45 (1982), no. 2, 245-273. MR647075

[AGG02] A. Arias, A. Gheondea, and S. Gudder, Fixed points of quantum operations, J. Math. Phys. 43 (2002), no. $12,5872-5881$. MR1939622

[BDH88] M. Baillet, Y. Denizeau, and J.-F. Havet, Indice d'une espérance conditionnelle, Compositio Math. 66 (1988), no. 2, 199-236. MR945550

[BE00] J. Böckenhauer and D. E. Evans, Modular invariants from subfactors: Type I coupling matrices and intermediate subfactors, Comm. Math. Phys. 213 (2000), no. 2, 267-289. MR1785458 (2001g:46142)

[BE98] - Modular invariants, graphs and $\alpha$-induction for nets of subfactors. I, Comm. Math. Phys. 197 (1998), no. 2, 361-386, available at arXiv:hep-th/9801171 MR1652746 (2000c:46121) 
[BE99] - Modular invariants, graphs and $\alpha$-induction for nets of subfactors. III, Comm. Math. Phys. 205 (1999), no. 1, 183-228, available at arXiv:hep-th/9812110 MR1706884 (2000j:46118)

[BEK00] J. Böckenhauer, D. E. Evans, and Y. Kawahigashi, Chiral structure of modular invariants for subfactors, Comm. Math. Phys. 210 (2000), no. 3, 733-784. MR1777347 (2001k:46097)

[BEK01] _ Longo-Rehren subfactors arising from $\alpha$-induction, Publ. Res. Inst. Math. Sci. 37 (2001), no. 1, 1-35. MR1815993 (2002d:46053)

[BEK99] _ On $\alpha$-induction, chiral generators and modular invariants for subfactors, Comm. Math. Phys. 208 (1999), no. 2, 429-487. MR1729094 (2001c:81180)

[BGL93] R. Brunetti, D. Guido, and R. Longo, Modular structure and duality in conformal quantum field theory, Comm. Math. Phys. 156 (1993), 201-219, available at funct-an/9302008v1

[BH95] W. R. Bloom and H. Heyer, Harmonic analysis of probability measures on hypergroups, de Gruyter Studies in Mathematics, vol. 20, Walter de Gruyter \& Co., Berlin, 1995. MR1312826

[BH96] D. Bisch and U. Haagerup, Composition of subfactors: new examples of infinite depth subfactors, Ann. Sci. École Norm. Sup. (4) 29 (1996), no. 3, 329-383. MR1386923 (97e:46080)

[Bis12] M. Bischoff, Models in boundary quantum field theory associated with lattices and loop group models, Comm. Math. Phys. 315 (2012), no. 3, 827-858. MR2981815

[Bis16a] _ , The relation between subfactors arising from conformal nets and the realization of quantum doubles, to appear in the Proceedings in honor of Vaughan F. R. Jones' 60th birthday conferences, 2016.

[Bis16b] _ A Remark on CFT Realization of Quantum Doubles of Subfactors: Case Index < 4, Lett. Math. Phys. 106 (2016), no. 3, 341-363. MR3462031

[BKL15] M. Bischoff, Y. Kawahigashi, and R. Longo, Characterization of $2 D$ rational local conformal nets and its boundary conditions: the maximal case, Doc. Math. 20 (2015), 1137-1184. MR3424476

[BKLR15] M. Bischoff, Y. Kawahigashi, R. Longo, and K.-H. Rehren, Tensor categories and endomorphisms of von Neumann algebras - with applications to quantum field theory, Springer Briefs in Mathematical Physics, vol. 3, Springer, Cham, 2015. MR3308880

[BKLR16] _ Phase Boundaries in Algebraic Conformal QFT, Comm. Math. Phys. 342 (2016), no. 1, 1-45. MR3455144

[Car04] S. Carpi, On the representation theory of Virasoro nets, Comm. Math. Phys. 244 (2004), no. 2, 261-284. MR2031030 (2005e:81128)

[Car98] _ Absence of subsystems for the Haag-Kastler net generated by the energy-momentum tensor in two-dimensional conformal field theory, Lett. Math. Phys. 45 (1998), no. 3, 259-267. MR1641204

[Cho74] M. D. Choi, A Schwarz inequality for positive linear maps on $C^{*}$-algebras, Illinois J. Math. 18 (1974), 565-574. MR0355615

[Con73] A. Connes, Une classification des facteurs de type III, Ann. Sci. École Norm. Sup.(4) 6 (1973), $133-252$.

[Con77] _ Periodic automorphisms of the hyperfinite factor of type II1, Acta Sci. Math. (Szeged) 39 (1977), no. 1-2, 39-66. MR0448101

[Con94] _ Non-commutative geometry, Academic Press, San Diego, 1994.

[DHR71] S. Doplicher, R. Haag, and J. E. Roberts, Local observables and particle statistics. I, Comm. Math. Phys. 23 (1971), 199-230. MR0297259 (45 \#6316)

[DM97] C. Dong and G. Mason, On quantum Galois theory, Duke Math. J. 86 (1997), no. 2, 305-321. MR1430435

[DMNO13] A. Davydov, M. Müger, D. Nikshych, and V. Ostrik, The Witt group of non-degenerate braided fusion categories, J. Reine Angew. Math. 677 (2013), 135-177. MR3039775

[DNO13] A. Davydov, D. Nikshych, and V. Ostrik, On the structure of the Witt group of braided fusion categories, Selecta Math. (N.S.) 19 (2013), no. 1, 237-269. MR3022755

[DR89a] S. Doplicher and J. E. Roberts, Endomorphisms of $C^{*}$-algebras, cross products and duality for compact groups, Ann. of Math. (2) 130 (1989), no. 1, 75-119. MR1005608

[DR89b] _ A new duality theory for compact groups, Invent. Math. 98 (1989), no. 1, 157-218. MR1010160 (90k:22005)

[DR90] - Why there is a field algebra with a compact gauge group describing the superselection structure in particle physics, Comm. Math. Phys. 131 (1990), no. 1, 51-107. MR1062748

[EG11] D. E. Evans and T. Gannon, The exoticness and realisability of twisted Haagerup-Izumi modular data, Comm. Math. Phys. 307 (2011), no. 2, 463-512. MR2837122 (2012m:17040)

[EG14] _ Near-group fusion categories and their doubles, Adv. Math. 255 (2014), 586-640. MR3167494

[EGNO15] P. Etingof, S. Gelaki, D. Nikshych, and V. Ostrik, Tensor categories, Mathematical Surveys and Monographs, vol. 205, American Mathematical Society, Providence, RI, 2015. MR3242743

[ENO10] P. Etingof, D. Nikshych, and V. Ostrik, Fusion categories and homotopy theory, Quantum Topol. 1 (2010), no. 3, 209-273. With an appendix by Ehud Meir. MR2677836 
[EP15] D. E. Evans and M. Pugh, Spectral measures for $G_{2}$, Comm. Math. Phys. 337 (2015), no. 3, $1161-1197$. MR3339174

[FFRS10] J. Fröhlich, J. Fuchs, I. Runkel, and C. Schweigert, Defect lines, dualities and generalised orbifolds, XVIth International Congress on Mathematical Physics, 2010, pp. 608-613. MR2730830

[FJ96] K. Fredenhagen and M. Jörß, Conformal Haag-Kastler nets, pointlike localized fields and the existence of operator product expansions, Comm. Math. Phys. 176 (1996), no. 3, 541-554.

[FRS04] J. Fuchs, I. Runkel, and C. Schweigert, TFT construction of RCFT correlators. III. Simple currents, Nuclear Phys. B 694 (2004), no. 3, 277-353. MR2076134 (2005e:81209)

[FRS89] K. Fredenhagen, K.-H. Rehren, and B. Schroer, Superselection sectors with braid group statistics and exchange algebras. I. General theory, Comm. Math. Phys. 125 (1989), no. 2, 201-226. MR1016869 (91c:81047)

[GF93] F. Gabbiani and J. Fröhlich, Operator algebras and conformal field theory, Comm. Math. Phys. 155 (1993), no. 3, 569-640.

[GL92] D. Guido and R. Longo, Relativistic invariance and charge conjugation in quantum field theory, Comm. Math. Phys. 148 (1992), no. 3, 521-551. MR1181069

[Haa87] U. Haagerup, Connes' bicentralizer problem and uniqueness of the injective factor of type $\mathrm{III}_{1}$, Acta Math. 158 (1987), no. 1-2, 95-148. MR880070 (88f:46117)

[HKL15] Y.-Z. Huang, A. Kirillov Jr., and J. Lepowsky, Braided tensor categories and extensions of vertex operator algebras, Comm. Math. Phys. 337 (2015), no. 3, 1143-1159. MR3339173

[HY00] T. Hayashi and S. Yamagami, Amenable tensor categories and their realizations as AFD bimodules, J. Funct. Anal. 172 (2000), no. 1, 19-75. MR1749868 (2001d:46092)

[ILP98] M. Izumi, R. Longo, and S. Popa, A Galois correspondence for compact groups of automorphisms of von Neumann algebras with a generalization to Kac algebras, J. Funct. Anal. 155 (1998), no. 1, 25-63. MR1622812 (2000c:46117)

[Izu00] M. Izumi, The Structure of Sectors Associated with Longo-Rehren Inclusions

I. General Theory, Comm. Math. Phys. 213 (2000), 127-179.

[Izu01] _ The structure of sectors associated with Longo-Rehren inclusions. II. Examples, Rev. Math. Phys. 13 (2001), no. 5, 603-674. MR1832764 (2002k:46161)

[Izu15] , A Cuntz algebra approach to the classification of near-group categories, arXiv preprint arXiv:1512.04288 (2015).

[Izu91] , Application of fusion rules to classification of subfactors, Publ. Res. Inst. Math. Sci. 27 (1991), no. 6, 953-994. MR1145672

[JMS14] V. F. R. Jones, S. Morrison, and N. Snyder, The classification of subfactors of index at most 5, Bull. Amer. Math. Soc. (N.S.) 51 (2014), no. 2, 277-327. MR3166042

[Jon80] V. F. R. Jones, Actions of finite groups on the hyperfinite type $\mathrm{II}_{1}$ factor, Mem. Amer. Math. Soc. 28 (1980), no. 237, v+70. MR587749

[JS97] V. Jones and V. S. Sunder, Introduction to subfactors, London Mathematical Society Lecture Note Series, vol. 234, Cambridge University Press, Cambridge, 1997. MR1473221 (98h:46067)

[Kad52] R. V. Kadison, A generalized Schwarz inequality and algebraic invariants for operator algebras, Ann. of Math. (2) 56 (1952), 494-503. MR0051442

[Kaw02] Y. Kawahigashi, Generalized Longo-Rehren subfactors and $\alpha$-induction, Comm. Math. Phys. 226 (2002), no. 2, 269-287. MR1892455

[KL04] Y. Kawahigashi and R. Longo, Classification of local conformal nets. Case c < 1., Ann. Math. 160 (2004), no. 2, 493-522.

[KLM01] Y. Kawahigashi, R. Longo, and M. Müger, Multi-Interval Subfactors and Modularity of Representations in Conformal Field Theory, Comm. Math. Phys. 219 (2001), 631-669, available at arXiv:math/9903104

[KO02] Jr. A. Kirillov and V. Ostrik, On a q-analogue of the McKay correspondence and the ADE classification of $\mathfrak{s l} 2$ conformal field theories, Adv. Math. 171 (2002), no. 2, 183-227. MR1936496 (2003j:17019)

[Lon03] R. Longo, Conformal Subnets and Intermediate Subfactors, Comm. Math. Phys. 237 (2003), 7-30, available at arXiv:math/0102196v2[math.0A].

[Lon79] _ Notes on algebraic invariants for noncommutative dynamical systems, Comm. Math. Phys. 69 (1979), no. 3, 195-207. MR550019 (80j:46108)

[Lon89] _ Index of subfactors and statistics of quantum fields. I, Comm. Math. Phys. 126 (1989), $217-247$.

[Lon94] _ A duality for Hopf algebras and for subfactors. I, Comm. Math. Phys. 159 (1994), no. 1, 133-150. MR1257245 (95h:46097)

[LR95] R. Longo and K.-H. Rehren, Nets of Subfactors, Rev. Math. Phys. 7 (1995), 567-597, available at arXiv: hep-th/9411077 
[LR97] R. Longo and J. E. Roberts, A theory of dimension, K-Theory 11 (1997), no. 2, 103-159, available at arXiv: funct-an/9604008v1. MR1444286 (98i:46065)

[Müg03a] M. Müger, From subfactors to categories and topology. I. Frobenius algebras in and Morita equivalence of tensor categories, J. Pure Appl. Algebra 180 (2003), no. 1-2, 81-157. MR1966524 (2004f:18013)

[Müg03b] _ From subfactors to categories and topology. II. The quantum double of tensor categories and subfactors, J. Pure Appl. Algebra 180 (2003), no. 1-2, 159-219. MR1966525 (2004f:18014)

[Müg03c] _ _ On the structure of modular categories, Proc. London Math. Soc. (3) 87 (2003), no. 2, 291-308. MR1990929

[Müg05] _ Conformal Orbifold Theories and Braided Crossed G-Categories, Comm. Math. Phys. 260 (2005), $727-762$

[Müg10] _ On superselection theory of quantum fields in low dimensions, XVIth International Congress on Mathematical Physics, 2010, pp. 496-503. MR2730815 (2012i:81165)

[NSZ03] C. P. Niculescu, A. Ströh, and L. Zsidó, Noncommutative extensions of classical and multiple recurrence theorems, J. Operator Theory 50 (2003), no. 1, 3-52. MR2015017

[Ocn01] A. Ocneanu, Operator algebras, topology and subgroups of quantum symmetry-construction of subgroups of quantum groups, Taniguchi Conference on Mathematics Nara '98, 2001, pp. 235-263. MR1865095 (2002j:57059)

[Ost03] V. Ostrik, Fusion categories of rank 2, Math. Res. Lett. 10 (2003), no. 2-3, 177-183. MR1981895

[Pas73] W. L. Paschke, Inner product modules over $B^{*}$-algebras, Trans. Amer. Math. Soc. 182 (1973), $443-468$. MR0355613

[Pop95] S. Popa, Classification of subfactors and their endomorphisms, CBMS Regional Conference Series in Mathematics, vol. 86, Published for the Conference Board of the Mathematical Sciences, Washington, DC; by the American Mathematical Society, Providence, RI, 1995. MR1339767 (96d:46085)

[Reh00] K.-H. Rehren, Canonical tensor product subfactors, Comm. Math. Phys. 211 (2000), no. 2, 395-406. MR1754521 (2001d:46093)

[Reh94] __ A new view of the Virasoro algebra, Lett. Math. Phys. 30 (1994), no. 2, 125-130. MR1264993 (95b:81194)

[Sun92] V. S. Sunder, $\mathrm{II}_{1}$ factors, their bimodules and hypergroups, Trans. Amer. Math. Soc. 330 (1992), no. 1, 227-256. MR1049618 (92f:46076)

[SW03] V. S. Sunder and N. J. Wildberger, Actions of finite hypergroups, J. Algebraic Combin. 18 (2003), no. 2, 135-151. MR2002621

[SW86] A. N. Schellekens and N. P. Warner, Conformal subalgebras of Kac-Moody algebras, Phys. Rev. D (3) 34 (1986), no. 10, 3092-3096. MR867023

[Tak03] M. Takesaki, Theory of operator algebras. III, Encyclopaedia of Mathematical Sciences, vol. 127, SpringerVerlag, Berlin, 2003. Operator Algebras and Non-commutative Geometry, 8. MR1943007 (2004g:46080)

[Tak72] _ Conditional expectations in von Neumann algebras, J. Functional Analysis 9 (1972), 306-321. MR0303307

[TY98] D. Tambara and S. Yamagami, Tensor categories with fusion rules of self-duality for finite abelian groups, J. Algebra 209 (1998), no. 2, 692-707. MR1659954

[Was90] A. Wassermann, Subfactors arising from positive energy representations of some infinite dimensional groups, unpublished notes (1990).

[Wil97] N. J. Wildberger, Duality and entropy of finite commutative hypergroups and fusion rule algebras, J. London Math. Soc. (2) 56 (1997), no. 2, 275-291. MR1489137

[Xu00] F. Xu, Algebraic orbifold conformal field theories, Proc. Nat. Acad. Sci. U.S.A. 97 (2000), no. 26, 14069, available at arXiv:math/0004150v1 [math.QA]

[Xu05] _ Strong additivity and conformal nets, Pacific J. Math. 221 (2005), no. 1, 167-199. MR2194151 (2007b:81132)

[Xu14] _ On intermediate conformal nets, J. Reine Angew. Math. 692 (2014), 125-151. MR3274549

[Xu98a] _ Applications of braided endomorphisms from conformal inclusions, Internat. Math. Res. Notices 1 (1998), 5-23. MR1601870

[Xu98b] _ New braided endomorphisms from conformal inclusions, Comm. Math. Phys. 192 (1998), no. 2, 349-403. MR1617550

Current address: Vanderbilt University, Department of Mathematics, 1326 Stevenson Center, Nashville, TN 37240 , USA

E-mail address: marcel.bischoff@vanderbilt.edu 